MedKlinIntensivmedNotfmed2021·116(Suppl2):S47-S72 https://doi.org/10.1007/s00063-021-00827-y Online publiziert: 18. Mai 2021

(c) Springer Medizin Verlag GmbH, ein Teil von Springer Nature 2021

\title{
Abstracts der 52. Gemeinsamen Jahrestagung der DGIIN und ÖGIAIN
}

\section{Kommunikation und Qualifikation}

\author{
online, 16.-18. Juni 2021
}

\section{Infektiologie//mmunologie}

\section{P01}

\section{Einfluss von Therapeutischem Drug Monitoring (TDM) von Piperacillin auf die Sepsis-assoziierte Organdysfunktion von Patienten mit Sepsis: eine randomisiert kontrollierte Studie}

S. Hagel, A. Brinkmann ${ }^{1}$, F. Bach ${ }^{2}$, M. Bauer ${ }^{3}$, T. Brenner ${ }^{4}$, D. Witzke ${ }^{2}$, D. Weismann ${ }^{5}$, M. Weigand ${ }^{6}$, S. Kluge ${ }^{7}$, A. Nierhaus ${ }^{7}$, D. Jarczak ${ }^{7}$, G. Michels ${ }^{8}$, T. Annecke ${ }^{9}$, A. Hohn ${ }^{10}$, C. Müller ${ }^{11}$, T. Lehmann ${ }^{12}$, M. Kiehntopf ${ }^{13}$, O. Frey ${ }^{14}$, M. Pletz

Center for Sepsis Control and Care, Institut für Infektionsmedizin und Krankenhaushygiene, Universitätsklinikum Jena, Jena; ${ }^{1}$ Klinik für Anästhesie, operative Intensivmedizin und spezielle Schmerztherapie, Klinikum Heidenheim, Heidenheim; ${ }^{2}$ Klinik für Anästhesiologie, Intensiv-, Transfusions-, Notfallmedizin und Schmerztherapie (AINS), Evangelisches Klinikum Bethel, Bielefeld; ${ }^{3}$ Klinik für Anästhesiologie und Intensivtherapie, Universitätsklinikum, Friedrich-Schiller-Universität Jena, Jena; ${ }^{4}$ Klinik für Anästhesiologie und Intensivmedizin, Universitätsklinikum Essen, Essen; ${ }^{5}$ Schwerpunkt Internistische Notfall- und Intensivmedizin, Medizinische Klinik und Poliklinik I, Universitätsklinikum Würzburg, Würzburg; ${ }^{6}$ Klinik für Anästhesiologie, Medizinische Fakultät, RuprechtKarls-Universität Heidelberg, Heidelberg; ${ }^{7}$ Klinik für Intensivmedizin, Zentrum für Anästhesiologie und Intensivmedizin, Universitätsklinikum Hamburg-Eppendorf, Hamburg; ${ }^{8}$ Klinik für Akut- und Notfallmedizin, St.Antonius-Hospital, Eschweiler; ${ }^{9} \mathrm{Klinik}$ für Anästhesiologie und operative Intensivmedizin, Krankenhaus Köln-Merheim, Klinikum der Universität Witten/Herdecke, Köln; ${ }^{10}$ Klinik für Anästhesiologie und operative Intensivmedizin, Kliniken Maria Hilf GmbH, Mönchengladbach; ${ }^{11}$ Zentrum für Pharmakologie, Uniklinikum Köln, Köln; ${ }^{12}$ Institut für Medizinische Statistik, Informatik und Dokumentation, Friedrich-Schiller-Universität Jena, Jena; ${ }^{13}$ Institut für Labormedizin, Universitätsklinikum Jena, Jena; ${ }^{14}$ Apotheke, Kliniken Landkreis Heidenheim gGmbH, Heidenheim;

Hintergrund: Eine inadäquate Dosierung von Antiinfektiva kann zu einem schlechteren Behandlungsergebnis bei Patienten mit Sepsis führen. Ziel der Studie war es zu untersuchen, ob eine Dosisoptimierung mittels Therapeutischem Drug Monitoring (TDM) von Piperacillin einen Einfluss auf die sepsisbedingte Organdysfunktion hat.

Methode: In diese randomisiert, kontrollierte Studie wurden Erwachsene mit schwerer Sepsis od. septischem Schock von Jan 2017 bis Dez 2019 in 10 Kliniken in Deutschland eingeschlossen. Die Dosierung des kontinuierlich verabreichten Piperacillin/Tazobactam (TZP) wurde in der Interventionsgruppe mittels täglich durchgeführtem TDM von Piperacillin gesteuert. In der Kontrollgruppe wurde TZP mit einer Tagesgesamtdosis von 13,5 g (bei normaler Nierenfunktion) kontinuierlich verabreicht. Das pharmakokinetisch/-dynamische Ziel war $100 \%$ fT $>4 \times$ MHK des nachgewiesenen Erregers, bzw. von Pseudomonas aeruginosa (MHK 16 mg/L) bei unbekanntem Erreger. Die primäre Zielgröße war der mittlere totale SOFA-Score bis Tag 10 (Sequential Organ Failure Assessment).

Ergebnisse: Insgesamt wurden 124 Patienten in der Kontroll- und $125 \mathrm{~Pa}$ tienten in der TDM-Gruppe analysiert. Das mittlere Alter betrug 66,2 Jahre, $74,3 \%$ der Patienten hatten einen septischen Schock. Es zeigte sich kein Unterschied im mittleren SOFA-Score zwischen den beiden Gruppen (TDM 7,9 Punkte vs. kein TDM 8,2, $\triangle \mathrm{SOFA}=0,3,95 \%$ CI 0,4-1,0, $p=0,39)$. Auch zeigte sich kein Unterschied in den weiteren sekundären Endpunkten, inkl. 28-Tage Sterblichkeit (TDM 21,6\% vs. kein TDM $25,8 \%, p=0,44)$. Das PK/PD-Ziel wurde häufiger in der TDM-Gruppe erreicht. Bereinigt um den Schweregrad der Erkrankung war die an Tag 1 nach Randomisierung gemessene Piperacillin-Konzentration mit der Sterblichkeit an Tag 28 assoziiert. Die Sterblichkeit war bei Patienten mit einer Konzentration von $>96$ mg/L (35/104) signifikant höher als bei einer Konzentration von $32-64 \mathrm{mg} / \mathrm{L}$ (4/48) (33,7\% vs. $8,3 \%$, OR 4,21, $95 \%$ CI 1,42-12,48, $p=0,01)$ bzw. $64-96$ mg/L (12/61) (33,7 \% vs. 19,7\%, OR 2,53, $95 \%$ CI 1,10-5,81, $p=0,03)$. Die Sterblichkeit war ebenfalls höher bei einer Piperacillin-Konzentration von $<32 \mathrm{mg} / \mathrm{l}(4 / 26)$ als bei einer Konzentration von $32-64 \mathrm{mg} / \mathrm{L}$ ( $15,4 \%$ vs. $8,3 \%$, OR 1,84, $95 \%$ CI $0,38-8,81, p=0,45)$.

Zusammenfassung: Eine TDM-gesteuerte Therapie hatte keinen Einfluß auf die sepsisbedingte Organdysfunktion. Patienten, die das PK/PD-Ziel nicht erreicht haben (d.h. über- oder unterschritten), hatten eine höhere Sterblichkeit (• Abb. 1).

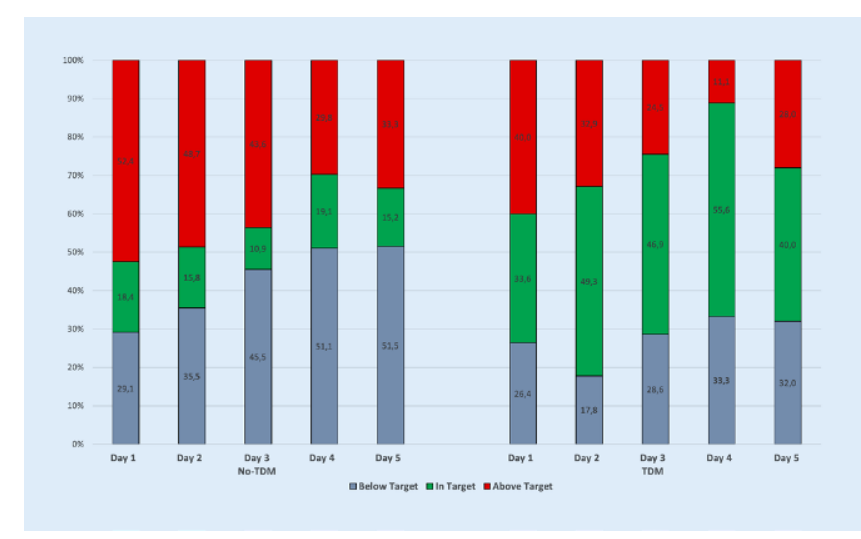

Abb. 1 | P01 4 


\section{P02}

\section{Bakteriämien bei intensivpflichtigen COVID-19 Patienten}

\section{Bota, G. Janssen, M. Keuchel, P. Baltes}

Klinik für Innere Medizin, Agaplesion Bethesda Krankenhaus Bergedorf, Hamburg;

Hintergrund: Ko-Infektionen gelten bei COVID-19 Patienten als selten. Bisherige Studien haben vor allem Ventilator-assoziierte Pneumonien untersucht. Unser Fokus lag auf dem Auftreten von Bakteriämien und Candidämien bei intensivpflichtigen Patienten mit COVID-19.

Methode: Wir schlossen retrospektiv alle Patienten ein, die zwischen März 2020 und Februar 2021 auf die Intensivstation eines Schwerpunktversorgers mit der Hauptdiagnose COVID-19 aufgenommen wurden. Die Infektion mit SARS-CoV2 wurde mittels PCR nachgewiesen. Patienten ohne bestätigte Indikation zur Intensivtherapie oder Aufenthalt (• Abb. 1 und 2).

Ergebnisse: Es wurden 260 Blutkulturpaare (BK) bei 46/56 Patienten abgenommen mit 15 positiven Befunden. Die Entnahme erfolgte während des Aufenthaltes bis zu sieben Mal, entsprechend 387 BK pro $1000 \mathrm{Be}-$ handlungstage. Bei 2 Patienten war die BK bei Aufnahme positiv, bei 13 erst im Verlauf. Koagulase-negative Staphylokokken (KNS) wurden bei 8 Patienten nachgewiesen, diese wurden in 7 Fällen als Kontamination und

\begin{tabular}{ll}
\hline Patienten & $N=56$ \\
\hline Männliches Geschlecht & $39(71 \%)$ \\
\hline Alter (Jahre) (mean \pm SD) & $68( \pm 14,7)$ \\
\hline SOFA Score (höchster Wert) (median $\pm S D)$ & $7( \pm 4,22)$ \\
\hline Mechanische Beatmung & $34(60,1 \%)$ \\
\hline Nierenersatztherapie & $6(10,7 \%)$ \\
\hline Aufenthaltsdauer ICU (Tage) (mean $\pm S D)^{1}$ & $12,3( \pm 12,3)$ \\
\hline Krankenhausaufenthalt (Tage) (mean $\pm S D)^{1}$ & $18,44( \pm 16,6)$ \\
\hline Mortalität (hausintern) & $34,9 \%$ \\
\hline Verlegungen ${ }^{2}$ & 8 \\
\hline Zentraler Venenkatheter (ZVK) & $50,0 \%$ \\
\hline Parenterale Ernährung & $46,4 \%$ \\
\hline Anzahl Blutkulturen & 260 \\
\hline Blutkulturen pro 1000 Behandlungstage & 387 \\
\hline Positive Blutkulturen & $34(13,1 \%)$ \\
$\quad-\quad$ Davon infektiologisch relevant & $22(8,5 \%)$ \\
\hline Patienten mit positiver Blutkultur & $15(26,8 \%)$ \\
$\quad$ Davon relevant & $9(16,1 \%)$ \\
\hline 14 Patienten zum Erhebungszeitpunkt noch nicht aus der stationären Behandlung entlassen \\
28 Patienten in auswärtige Klinik verlegt (5 ECMO, 1 Gefäßintervention, 2 Weaning) /
\end{tabular}

Abb. 1 | P02 $\triangle$ Demografie und klinische Details

\begin{tabular}{ll}
\hline Erreger & \\
\hline Koagulase negative Staphylokokken & 8 \\
\hline Staphylococcus aureus & 1 \\
\hline Streptococcus pneumoniae & 3 \\
\hline Enterococcus faecalis & 1 \\
\hline Enterococcus faecium & 1 \\
\hline Klebsiella pneumoniae & 1 \\
\hline Klebsiella oxytoca & 1 \\
\hline Serratia marcescens & 1 \\
\hline Candida albicans & 1 \\
\hline & 1 \\
\hline Infektfokus: & \\
\hline Harnwegsinfektionen & 2 \\
\hline Ventilator assoziierte Pneumonie & 1 \\
\hline Ambulant erworbene Pneumonie & 1 \\
\hline Venenkatheterinfektion & 5 \\
\hline
\end{tabular}

Abb. 2 | P02 $\Delta$ Kultureller Nachweis und Infektionsfokus einmal als KNS Katheterinfektion gewertet. Bei weiteren Katheterinfektionen wurden Staphylococcus aureus $(n=3)$, Enterococcus faecium und faecalis (jeweils $n=1$ ) und Candida albicans $(n=1)$ nachgewiesen. In einem Fall lag eine Mischinfektion mit Entero- und Staphylokokken vor. Zwei Patienten erlitten eine weitere Blutstrominfektion.

Gramnegative Bakteriämien waren Folge einer ventilator-assoziierten Pneumonie $(n=1)$ und Harnwegsinfektionen $(n=2)$.

Patienten mit einer relevanten positiven Blutkultur waren schwerer erkrankt (SOFA 11,7 P vs. 7P, $p=0,0014$ ), bedurften einer längeren Intensivtherapie (ICU $26,7 \mathrm{~d}$ vs $9,7 \mathrm{~d}, p=0,0005$ ) und waren länger hospitali-

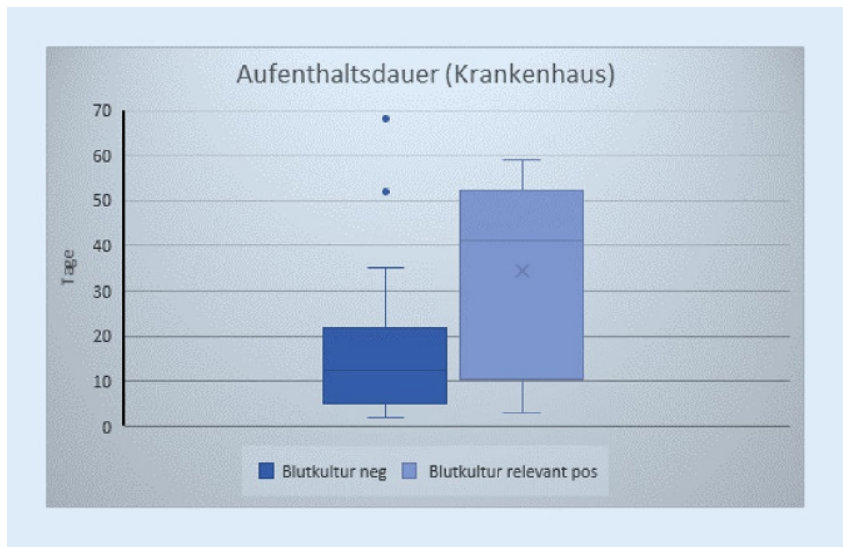

Abb. 3 | P02 $\triangle$

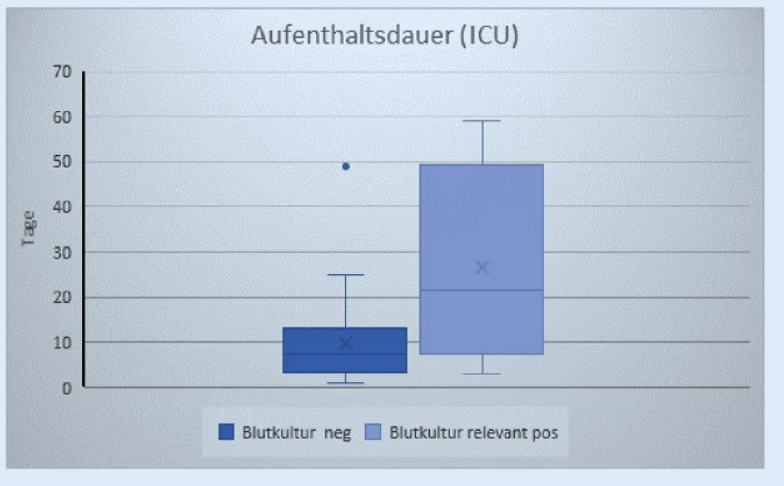

Abb. 4|P02 A

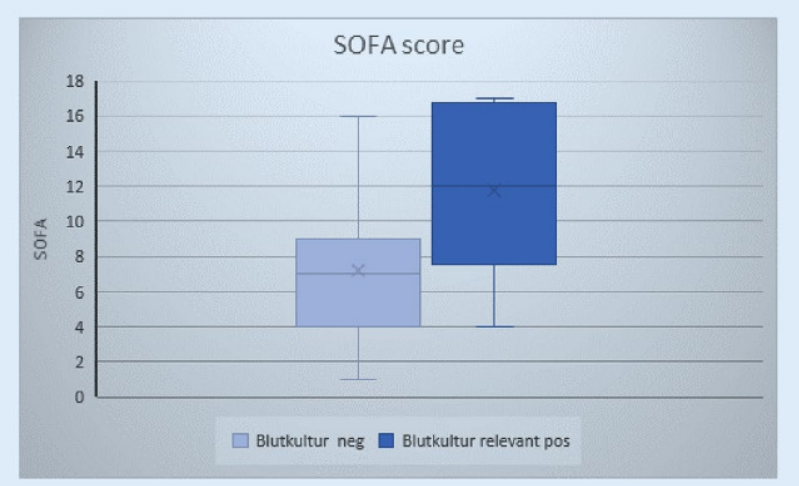

Abb. 5 |P02 A 


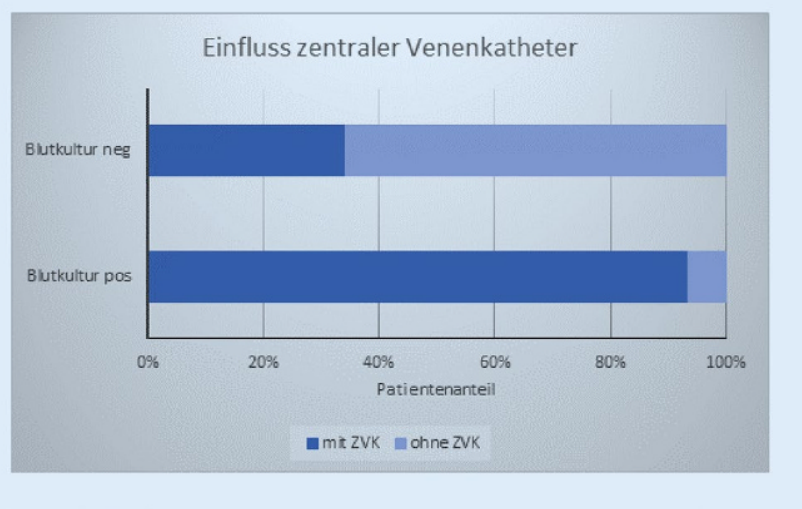

Abb. 6|P02 $\triangle$

siert (34,5d vs. $16,5 \mathrm{~d} ; p=0,007)$. Ein Mortalitätsunterschied ( $62,5 \%$ vs. $29,4 \%$ ) war, ebenso wie eine häufigere Beatmungspflichtigkeit (20,6\% vs. $9,1 \%)$ und eine Nierenersatztherapie, statistisch nicht signifikant. Zentrale Venenkatheter (ZVK) korrelierten ebenso wie eine parenterale Ernährung (PN) mit vermehrtem Auftreten (ZVK: $p=0,0065$; PN: $p=0,0085$; - Abb. 3, 4, 5 und 6).

Zusammenfassung: Bakteriämien traten bei intensivpflichtigen COVID-19 Patienten überwiegend als nosokomiale Infektionen auf und waren mit einem ZVK und parenteraler Ernährung assoziiert. Sie korrelierten mit einer höheren Krankheitsschwere sowie einem verlängerten Intensiv- und Krankenhausaufenthalt.

\section{P03}

\section{Pharmakokinetik von Cefiderocol bei kritisch kranken Patienten}

C. König, H. Rohde ${ }^{1}$, A. Both ' ', A. Röhr ${ }^{2}$, O. Frey ${ }^{2}$, S. Kluge ${ }^{3}$, D. Wichmann ${ }^{3}$ Krankenhausapotheke, Universitätsklinikum Hamburg-Eppendorf, Hamburg; ${ }^{1}$ Institut für Mikrobiologie, Virologie und Hygiene, Universitätsklinikum Hamburg-Eppendorf, Hamburg; ${ }^{2}$ Apotheke, Kliniken Landkreis Heidenheim gGmbH, Heidenheim; ${ }^{3}$ Klinik für Intensivmedizin, Zentrum für Anästhesiologie und Intensivmedizin, Universitätsklinikum Hamburg-Eppendorf, Hamburg;

Hintergrund: Cefiderocol ist ein neues Siderophor-Cephalosporin mit einer breiten Wirksamkeit gegen mutliresistente (MDR) Gram-negative Bakterien inklusive P. aeruginosa, A. baumannii und S. maltophilia. Derzeit gibt es keine oder kaum pharmakokinetische Daten insbesondere für kritisch kranke Patienten mit akutem Nierenversagen (ANV), kontinuierlichem Nierenersatzverfahren (CRRT) und Cytokin-Adsorber Therapie. Methode: Um die individuelle Pharmakokinetik von Cefiderocol in kritisch kranken Patienten mit septischem Schock und extrakorporalen Therapien (CRRT) einschätzen und untersuchen zu können, wurde ein Therapeutisches Drug Monitoring (TDM) etabliert. Fünf Patienten mit unterschiedlichem Grad an ANV, Bedarf an CRRT und Cytokin-Adsorber Therapie wurden auf Grund von Infektionen mit MDR Pseudomonas aeruginosa und Acinetobacter baumanni mit Cefiderocol behandelt und ein TDM durchgeführt. Auf Basis der gemessenen Cefiderocol Talspiegel, wurden die Dosierungen an ein Ziel von $4 \times$ MHK für $100 \%$ des Dosisintervalls angepasst. Zudem wurde während der Therapie bei nachgewiesenen Isolaten ein mikrobiologisches Monitoring bezüglich der Entstehung von Resistenzen durchgeführt.

Ergebnisse: Kritisch kranke Patienten mit ANV und CRRT zeigten Cefiderocol Konzentrationen von $4 \times \mathrm{MHK}$ während des gesamten Dosisintervalls mit einer reduzierten Dosis von $3 \times 1 \mathrm{~g}$ Cefiderocol als prolongierte Infusion. EIn Patient mit zunächst nur moderat eingerschränkter Nierenfunktion (GFR ca. $67 \mathrm{ml} / \mathrm{min}$ ) zeigte eine deutliche Verlängerung der Halbwertszeit auf mehr als $8 \mathrm{~h}$. Die Hinzunahme eines Cytokin-Adsorbers zur CRRT resultierte in Cefiderocol Konzentrationen von $1 \times \mathrm{MHK}$ am
Ende des Dosisintervalls unter einer Dosierung von $3 \times 2 \mathrm{~g}$. Während der Behandlung konnte keine Resistenzentwicklung gegen Cefiderocol festgestellt werden.

Zusammenfassung: Cefiderocol zeigt eine veränderte und variable Pharmakokinetik bei kritisch kranken Patienten mit ANV und CRRT. Insgesamt konnten außreichend hohe Arzneistoffkonzentrationen mit den vom Hersteller empfohlenen Dosierungen erreicht werden. Von besonderer Bedeutung sind die erniedrigten Cefiderocol Konzentrationen während der zusätzlichen Cytokin-Adsorber Therapie. Hier ist zum Monitoring und zur Optimierung der Therapie ein zeitnahes TDM empfehlenswert, um erniedrigten Spiegeln frühzeitig entgegen zu wirken.

\section{P04}

\section{Identification of Novel Sublingual Parameters to Analyze and Diagnose Microvascular Dysfunction in Sepsis: The NOSTRADAMUS Study}

\author{
A. Rovas, J. Sackarnd ' ${ }^{\text {, J. Rossaint }}{ }^{2}$, S. Kampmeier ${ }^{3}$, H. Pavenstädt ${ }^{4}$, H. Vink ${ }^{5}$, \\ P. Kümpers ${ }^{4}$
}

Allg. Innere Medizin sowie Nieren- und Hochdruckkrankheiten und Rheumatologie, Medizinische Klinik D, Westfälische Wilhelms-Universität Münster, Münster; ${ }^{1}$ Department für Kardiologie und Angiologie, Universitätsklinikum, Westfälische Wilhelms-Universität Münster, Münster; ${ }^{2}$ Klinik für Anästhesiologie, operative Intensivmedizin und Schmerztherapie, Universitätsklinikum, Westfälische Wilhelms-Universität Münster, Münster; ${ }^{3}$ Institut für Hygiene, Universitätsklinikum, Westfälische Wilhelms-Universität Münster, Münster; ${ }^{4}$ Allg. Innere Medizin und Notaufnahme sowie Nierenund Hochdruckkrankheiten und Rheumatologie, Medizinische Klinik D, Westfälische Wilhelms-Universität Münster, Münster; ${ }^{5}$ Kardiovaskuläres Forschungsinstitut, Universität Maastricht, Maastricht/NL;

Objective: The availability of handheld, non-invasive sublingual video-microscopes allows for visualization of the microcirculation in critically ill patients. Recent studies demonstrate that reduced numbers of blood-perfused microvessels and increased penetration of erythrocytes into the endothelial glycocalyx are essential components of microvascular dysfunction. The aim of this study was to identify novel microvascular variables to determine the level of microvascular dysfunction in sepsis and its relationship with clinical variables.

Method: This observational, prospective, cross-sectional study included 51 participants, of which 34 critically ill sepsis patients were recruited from intensive care units of a university hospital. 17 healthy volunteers served as controls. All participants underwent sublingual videomicroscopy by sidestream darkfield imaging. A new developed version of the Glycocheck ${ }^{\mathrm{ma}}$ software was used to quantify vascular density, perfused boundary region (PBR-an inverse variable of endothelial glycocalyx dimensions), red blood cell (RBC) velocity, RBC content and blood flow in sublingual microvessels with diameters between 4 and $25 \mu \mathrm{m}$.

Results: A detailed analysis of adjacent diameter-classes ( $1 \mu \mathrm{m}$ each) of vessels between 4 and $25 \mu \mathrm{m}$ revealed a severe reduction of vascular density in very small capillaries $(5-7 \mu \mathrm{m})$, which correlated with markers of sepsis severity. Analysis of RBC velocity (VRBC) revealed a strong dependency between capillary and feed vessel $\mathrm{VRBC}_{\mathrm{VRC}}$ in sepsis patients $\left(\mathrm{R}^{2}=0.63, p<0.0001\right)$ but not in healthy controls $\left(\mathrm{R}^{2}=0.04, p=0.43\right)$, indicating impaired capillary (de-)recruitment in sepsis. This finding enabled the calculation of capillary recruitment and dynamic capillary blood volume $\left(\mathrm{CBV}_{\text {dynamic }}\right)$. Moreover, adjustment of PBR to feed vessel ${ }_{\mathrm{VRBC}}$ further improved discrimination between sepsis patients and controls by about $50 \%$. By combining these dynamic microvascular and glycocalyx variables we developed the microvascular health score $\left(\mathrm{MVHS}_{\mathrm{dynamic}}{ }_{\mathrm{Tn}}\right)$, which decreased from 7.4 [4.6-8.7] in controls to 1.8 [1.4-2.7] in sepsis patients $(p<0.0001)$ and correlated with sepsis severity.

Conclusion: We introduce new important diameter-specific quantification and differentiated analysis of RBC kinetics, a key to understand microvascular dysfunction in sepsis. $\mathrm{MVHS}_{\text {dynamic }}$, which has a broad bandwidth to detect microvascular (dys-) function, might serve as a valuable tool to detect microvascular impairment in critically ill patients. 
P05

\section{Characteristics and outcome of patients with exacerbated systemic rheumatic diseases referred to the intensive care unit - a retrospective data analysis}

M. Schneeweiß-Gleixner, C. Hillebrand ', S. Jaksits ', G. Heinz ${ }^{2}$, G. Sengölge ${ }^{3}$ T. Staudinger ${ }^{4}$, M.-P. Winter ${ }^{2}$, C. Zauner, D. Aletaha ${ }^{1}$, K. Markstaller ${ }^{5}$, M. Trauner, K. Machold ', P. Schellongowski ${ }^{4}$, M. Bécède ${ }^{1}$

Abteilung für Gastroenterologie und Hepatologie, Klinik für Innere Medizin III, Medizinische Universität Wien, Wien; ${ }^{1}$ Abteilung für Rheumatologie, Klinik für Innere Medizin III, Medizinische Universität Wien, Wien $/ \mathrm{A} ;{ }^{2}$ Abteilung für Kardiologie, Klinik für Innere Medizin II, Medizinische Universität Wien, Wien $/ \mathrm{A} ;{ }^{3}$ Abteilung für Nephrologie und Dialyse, Klinik für Innere Medizin III, Medizinische Universität Wien, Wien $/ \mathrm{A}^{*}{ }^{4}$ Intensivstation 13i2, Universitätsklinik für Innere Medizin I, Medizinische Universität Wien, Wien/A; ${ }^{5}$ Universitätsklinik für Anästhesie, Allgemeine Intensivmedizin und Schmerztherapie, Medizinische Universität Wien, Wien/A;

Objective: Despite tremendous advances in the treatment of patients with systemic rheumatic diseases (SRD), affected patients are at high risk for admission to the intensive care unit (ICU). This is mostly due to infections, disease related complications or drug side effects. Data concerning these critically ill patients are limited to few retrospective studies on heterogenous populations. Primary aim of our study is to report a descriptive analysis on characteristics and outcome of our single center cohort of patients who were admitted to the ICU due to exacerbation of their underlying SRD.

Method: Patients were included retrospectively between 01.01 .2012 and 31.12.2020 at eleven ICU wards at the Vienna General Hospital. Potential eligible patients were identified with the AKH-Information-Management (AKIM) EDV tool by selecting ICD-10 coded diagnoses. Results are reported by using median and interquartile ranges.

Results: In total, 45 SRD patients ( $64.4 \%$ women with a median age of 43 years [interquartile range (IQR) 29-64 years]) were included. The median ICU length of stay was 13 days (IQR 8-20 days). Connective tissue diseases $(42.2 \%)$ and vasculitides (42.2\%) accounted for the most common SRDs observed. 23 patients (51.1\%) already received immunosuppression prior to ICU admission. The leading cause for ICU admission was respiratory insufficiency $(55.6 \%)$, followed by shock (15.6\%) and neurological impairment (8.9\%). Median SOFA-, SAPSII- and APACHEII-Score on day 1 were 9 (IQR6-11), 34 (26.8-42 and 16 (13.8-25.5), respectively. In total $77.8 \%$ of all patients required mechanical invasive ventilation (MIV) with a median length of MIV of 12 days (IQR 5.5-21.5 days). Furthermore, $75.6 \%$ of all patients received vasopressor therapy, in $44.4 \%$ renal replacement therapy was conducted. Extracorporeal membrane oxygenation (ECMO) was performed in 10 of 45 patients $(22.2 \%)$. Overall ICU mortality was $24.4 \%$, the 6-month mortality was $33.3 \%$. ICU readmission was required in 9 of 45 patients $(20 \%)$. ECMO patients had a $40 \%$ survival rate at ICU discharge.

Conclusion: In conclusion, patients admitted to the ICU due to SRD exacerbation still represent a major clinical challenge. Connective tissues diseases and vasculitides are the most common rheumatic entities requiring ICU treatment. Respiratory failure is the leading cause of ICU admission, a fact that is also reflected by high rates of required MIV and ECMO therapy. The lack of consistent data highlights the need for further research concerning prognostic parameters and more specific guidelines in treating these critically ill patients.

\section{P06}

Plasma exchange with COVID-19 convalescent plasma in a patient with severe ANCA-associated vasculitis and COVID-19 pneumonia after Rituximab therapy

L. Beckmann, M. Kochanek, H. Hagmann ' , N. Julia' 2, V. di Cristanziano ${ }^{3}$, B. Böll, D. Eichenauer, A. Shimabukuro-Vornhagen, B. Gathoff', J. Garcia Borrega

Klinik für Innere Medizin I, Universitätsklinikum Köln, Köln; ${ }^{1}$ Nephrologie, Rheumatologie, Diabetologie und Allgemeine Innere Medizin, Klinik II für Innere Medizin, Universitätsklinikum Köln, Köln; ${ }^{2}$ Transfusionsmedizin, Universitätsklinikum Köln, Köln; ${ }^{3}$ Institut für Virologie, Unversitätsklinikum Köln, Köln;

Objective: Previous treatment with B-cell directed antibodies like Rituximab (RTX) is associated with poor prognosis in patients with severe COVID-19 infection. Patients with RTX-induced hypogammaglobulinemia and severe COVID-19 could therefore profit from treatment with COVID-19 convalescent plasma (CCP).

Method: We report a case of a 52-year old male patient admitted to the intensive care unit (ICU) with severe COVID-19 pneumonia and acute relapse of ANCA-associated vasculitis (AAV) 18 days after the first dose of RTX maintenance therapy.

Results: The patient presented with severe hypoxemia (pO2 $47.4 \mathrm{mmHG}$, 2102 flow via nasal canula), hemoptysis and fever. PCR for SARS-CoV-2 was positive both in nasopharyngeal swabs and peripheral blood. Elevated MPO-ANCA levels of $533 \mathrm{RE} / \mathrm{ml}$ confirmed relapse of ANCA-associated vasculitis. A chest CT-scan showed left mid-zone consolidation, resembling both patterns found in COVID peak stage 3 and pulmonary-renal syndrome associated with AAV. Laboratory results showed complete B cell depletion.

After admission, High-Flow Nasal Oxygen (HFNO)was initiated. Therapeutic plasma exchange (PLEX) was performed for five consecutive days, processing 4 liters plasma during each treatment and substituting CCP. Two units of high titer CCP were transfused at the end of each PLEX. The other plasma used in PLEX were low titer CCP. High dose prednisolone was administered from day 1-5, and $1000 \mathrm{mg}$ cyclophosphamide on day 6 .

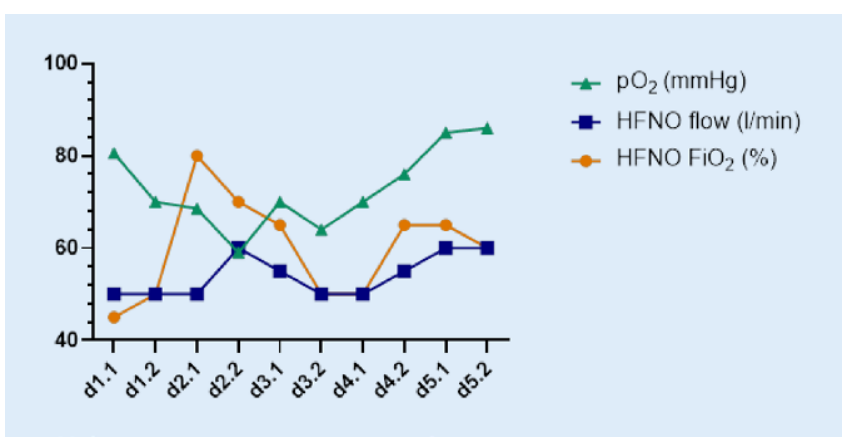

Fig. 1 | P06

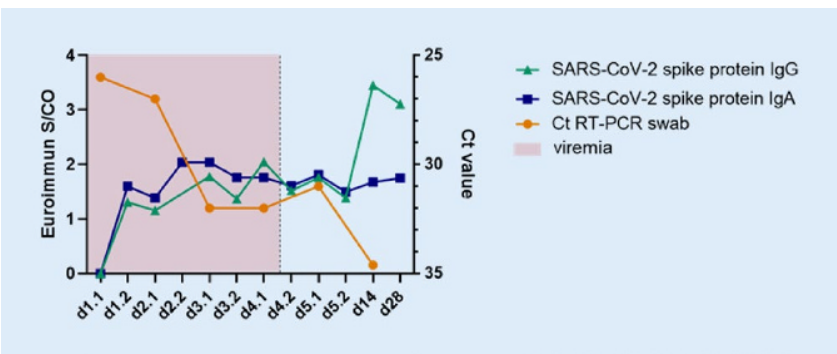

Fig. 2 | P06 $\triangle$ 


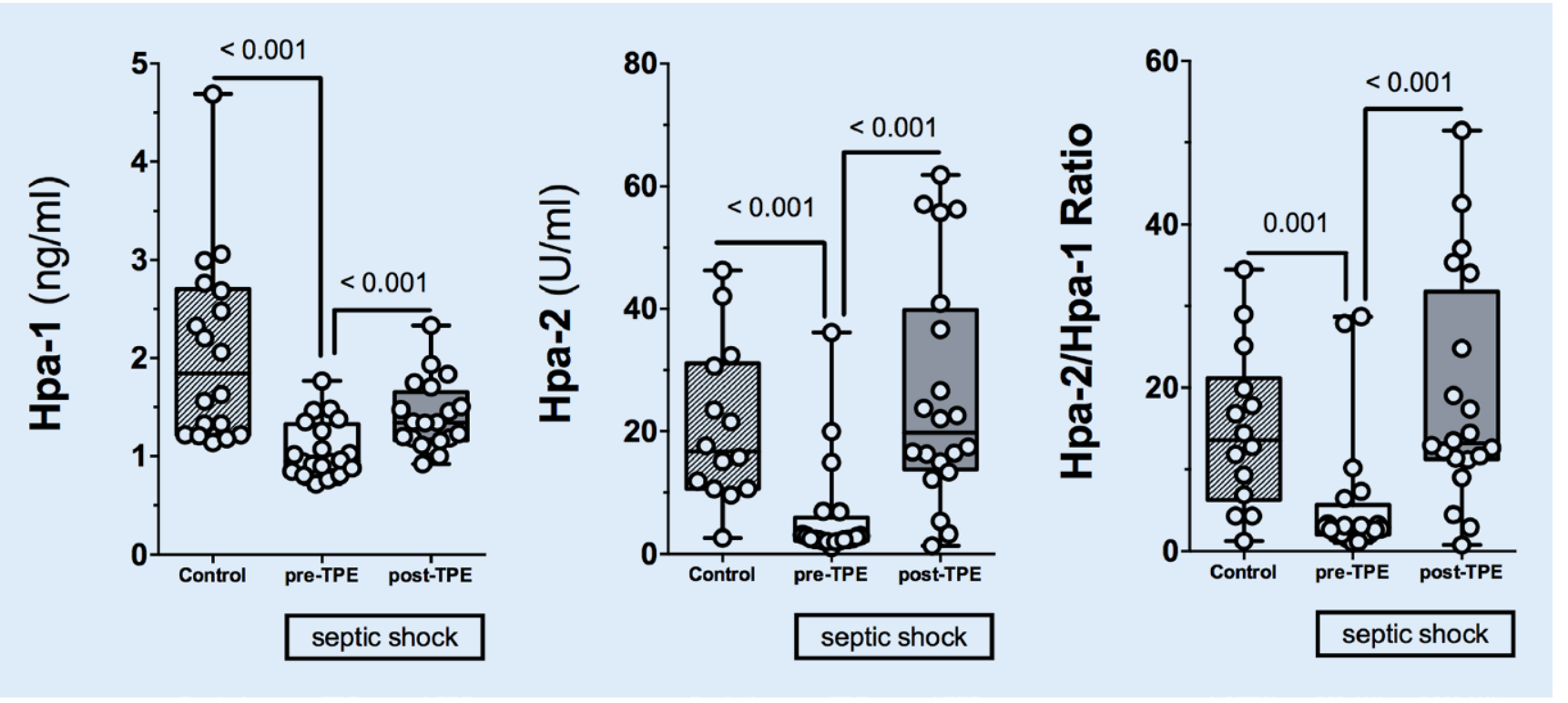

Fig. 1 | P07 $\Delta$

The patient's condition improved rapidly during PLEX. The patient could be weaned of the HFNO at day 6 (• Fig. 1).

Viremia lasted until day 4. COVID-19 specific antibodies (IgA and IgG) were positive after the first PLEX with a further increase of titer until day 28 (- Fig. 2).

ANCA and pro-inflammatory cytokines were cleared sufficiently. The patient was discharged on day 16 . The patient received $120 \mathrm{mg}$ prednisolone qd on discharge with continued tapering in the further course.

Conclusion: In the present case of acute AAV, severe COVID-19 pneumonia and depletion of B-cells after RTX, which implies insufficient humoral immune response to SARS-CoV-2, PLEX treatment with CCP as the substitute was performed. We achieved sufficient clearing of the ANCA and pro-inflammatory cytokines. In addition, we were able to generate a sufficiently high titer of anti-SARS-CoV-2 antibodies to control COVID-19 pneumonia. We show that therapeutic plasma exchange with CCP is safe and feasible, and it has led to a significant improvement of the clinical situation.

\section{P07}

\section{Effects of therapeutic plasma exchange on the endothelial glycocalyx in septic shock}

K. Stahl, U. C. Hillebrand ', Y. Kiyan ' , B. Seeliger ${ }^{2}$, J. J. Schmidt ' , H. Schenk', P.A. Gronski ', B. M. W. Schmidt ' , T. Welte ${ }^{2}$, M. Hoeper ${ }^{2}$, A. Sauer ${ }^{3}$, M. Wygrecka ${ }^{3}$, C. Bode ${ }^{4}$, H. Haller', S. David '

Klinik für Gastroenterologie, Zentrum für Innere Medizin,

Medizinische Hochschule Hannover, Hannover; ${ }^{1}$ Klinik für Nieren- und Hochdruckerkrankungen, Zentrum für Innere Medizin, Medizinische Hochschule Hannover, Hannover; ${ }^{2}$ Klinik für Pneumologie, Zentrum für Innere Medizin, Medizinische Hochschule Hannover, Hannover; ${ }^{3}$ Biochemisches Institut, Justus-Liebig-Universität, Gießen; ${ }^{4}$ Anästhesie und Intensivmedizin, Universitätsklinikum Bonn, Bonn;

Objective: Disruption of the endothelial glycocalyx (eGC) is observed in septic patients and its injury is associated with multiple-organ failure and inferior outcomes. Besides this biomarker function, increased blood concentrations of shedded eGC constituents might play a mechanistic role in septic organ failure. We hypothesized that therapeutic plasma exchange (TPE) against fresh frozen plasma might influence eGC related pathology. Method: We enrolled 20 norepinephrine dependent (NE $>0.4 \mathrm{mg} / \mathrm{kg} / \mathrm{min}$ ) patients with early septic shock (onset $<12 \mathrm{~h}$ ). Sublingual assessment of the eGC via sublingual sidestream darkfield (SDF) imaging was performed.

Plasma eGC degradation products such as heparan-sulfate (HS) and the eGC regulating enzymes, heparanase (Hpa)-1 and Hpa-2, were obtained before and after TPE. A 3D microfluidic flow assay was performed to examine the effect of TPE on eGC ex vivo. Results were compared to healthy controls.

Results: SDF demonstrated a marked decrease in eGC thickness in septic patients compared to healthy individuals $(p=0.001)$. Circulating HS levels were increased more than six-fold compared to controls and decreased significantly following TPE (controls: 16.9 (8-18.6) vs. septic patients before TPE: 105.8 (30.8-143.4) $\mu \mathrm{g} / \mathrm{ml}, p<0.001$; vs. after TPE: 70.7 (36.9-109.5) $\mu \mathrm{g} / \mathrm{ml}, p<0.001)$. The Hpa-2/Hpa- 1 ratio was markedly reduced in septic patients before TPE but normalized after TPE (controls: 13.6 (6.2-21.2)

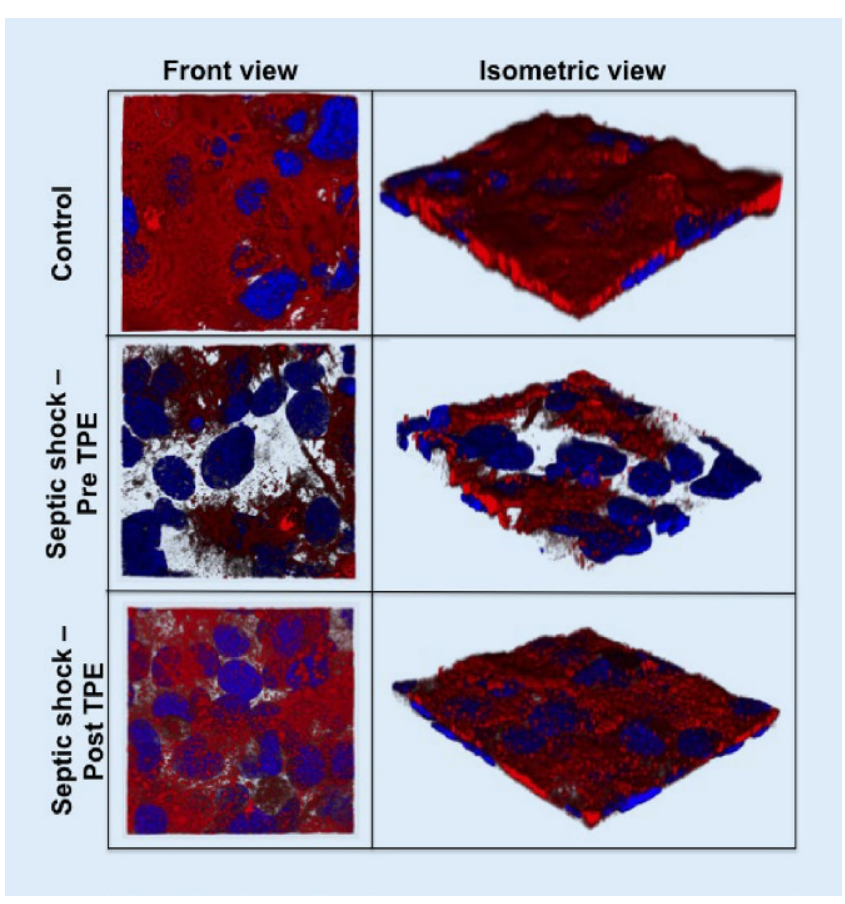

Fig. 2 | P07 A

Medizinische Klinik - Intensivmedizin und Notfallmedizin · Suppl 2 2021 
vs. septic patients at inclusion: $2.9(2.1-5.7), p=0.001$; vs. septic patients after TPE: 13.2 (11.2-31.8), $p<0.001$; • Fig. 1).

Ex vivo stimulation of endothelial cells with serum from septic patients induced eGC damage that could be attenuated with serum post TPE (- Fig. 2).

Conclusion: Septic shock results in profound degradation of the eGC and an acquired deficiency of the protective regulator Hpa-2. TPE removed potentially injurious eGC degradation products and partially attenuated Hpa-2 deficiency.

\section{P08}

\section{KADOIN - Kartenbasierte Kontaktnachverfolgung von COVID Indexpatienten}

G. Beutel, J. Wille?

Klinik für Hämatologie, Hämostaseologie, Onkologie und Stammzelltransplantation, Zentrum für Innere Medizin, Medizinische Hochschule Hannover, Hannover; 'Ubilabs, Hamburg;

Hintergrund: Die Kontaktpersonennachverfolgung ist eine wesentliche Säule zur Eindämmung der COVID-19 Pandemie. Diese Nachverfolgung wird derzeit überwiegend per Telefoninterview durch die Gesundheitsämter umgesetzt. Ziel des Forschungsprojekts KAODIN ist es, den Gesundheitsämter und Bürger*innen eine digitale Plattform zur Verfügung zu stellen, um Infektionsketten besser zu identifizieren und zu unterbrechen. Mit KADOIN sollen Machbarkeit, Akzeptanz und Effektivität der digitalen Kontaktpersonennachverfolgung evaluiert werden.

Methode: I) Verbesserte Dokumentation durch ein szenisches Gedächtnisprotokoll: Ein visuelles Kartensystem erleichtert Bürgerinnen sich an das Erlebte der letzten 14 Tage zu erinnern und die in dieser Zeit stattgehabten Kontakte besser wiedergeben zu können.

II) Semiquantitative Dateneingabe: Neben einer manuellen Dateneingabe können die ohnehin in vielen Smartphones gespeicherten Aufenthaltsorte importiert werden. Im Unterschied zu Tracing-Apps werden Orte und Wege für den Benutzer transparent dargestellt und können jederzeit nachbearbeitet oder gelöscht werden.

Ergebnisse: Die Anwendung KADOIN wurde bewusst als barrierefreie Browser-Anwendung und nicht als Smartphone-App entwickelt. Abgerufen wird die Anwendung über das Internet, der eigentliche Einsatz erfolgt ausschließlich lokal auf dem Endgerät. Neben der Dokumentation durch die Gesundheitsämter bietet die Anwendungssoftware die Möglichkeit, dass positiv getestete Personen die Dokumentation von Kontaktpersonen eigenständig und zeitlich unabhängig von behördlichen Öffnungszeiten durchführen können.

Unser Datenschutzkonzept beinhaltet, dass die Übermittlung der Daten nur nach aktive Freigabe des Nutzers erfolgt. Zu Fragen des Datenschutzes wurde die Projektgruppe durch den Bundesbeauftragten für den Daten-

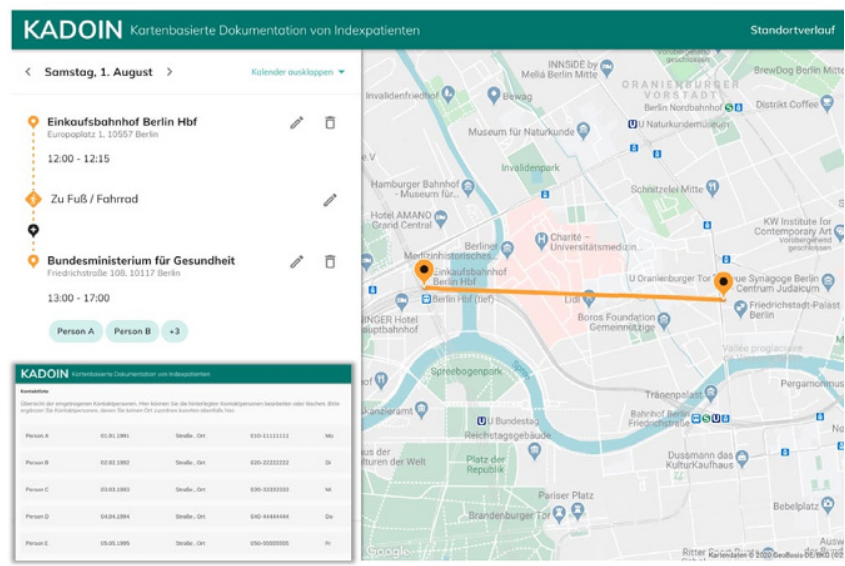

Abb. 1 | P08 schutz und die Informationsfreiheit und der lokalen Datenschutzbeauftragten und Ethikkommission beraten.

Zusammenfassung: Die Digitalisierung der Infektionsforschung hat innerhalb der letzten 12 Monate deutliche Impulse bekommen. Das Forschungsprojekt KADOIN zeigt beispielhaft, dass eine ergebnisorientierte Kooperation von Medizinern und IT-Spezialisten praxisnahe Lösungen initiieren können, um pandemische Szenarien besser kontrollieren und das Gesundheitssystem entlasten zu können. Studienergebnisse zur Machbarkeit, Akzeptanz und Effektivität von KADOIN sind aufgrund der Aktualität vorläufig und werden im Rahmen der Jahrestagung präsentiert (• Abb. 1).

\section{P09}

\section{An outpatient management strategy using remote digital monitoring and Coronataxi home care significantly reduces COVID-19 mortality rate}

\section{A. Lim, T. Hippchen, A. Welker ${ }^{1}$, M. Weigand ${ }^{2}$, U. Merle}

Gastroenterologie, Infektionskrankheiten, Vergiftungen, Klinik für Innere Medizin IV, Universitätsklinikum Heidelberg, Heidelberg; ${ }^{1}$ Gesundheitsamt Rhein-Neckar-Kreis, Heidelberg; ${ }^{2}$ Klinik für Anästhesiologie, Medizinische Fakultät, Ruprecht-Karls-Universität Heidelberg, Heidelberg;

Objective: The COVID-19 pandemic has caused great strain to healthcare systems and hospitals worldwide. Better outpatient management is required to prepare for future global pandemics. Hypoxemia due to COVID-19 occurs long before patients complain of subjective dyspnea and seek medical attention. Delayed medical attention and therapy likely result in higher rates of mechanical ventilation and mortality. Although many strategies to manage COVID-19 outpatients have been applied, there is currently limited information on their effectiveness. A new strategy combining remote digital monitoring and home quarantine visits for COVID-19 outpatients when indicated was implemented to optimize hospitalization rate and reduce mortality. The aim of this study is to measure the effectiveness of this new outpatient care system for COVID-19 in reducing mortality rate.

Method: This system consisted of a call-center to obtain patients' medical history and current symptoms, a delivery system to bring pulse oxymeters to the patients after initial contact with the call-center, a mobile application (Huma-Medopad ${ }^{\circ}$ ) for remote monitoring of self-reported symptoms and oxygen saturation thrice daily and a nursing team that makes additional visits in home quarantine, if indicated. Upon diagnosis, the regional health authority informed COVID-19 patients regarding this outpatient care program, especially encouraging participation from patients with known risk factors for severe COVID-19. Patients keyed in their peripheral oxygen saturation, heart rate, blood pressure, temperature and symptom progression into the app (Huma-Medopad ${ }^{\star}$ ) regularly. A physician remotely monitored these data, advised patients accordingly and initiated additional home care visits with blood analysis at their discretion. The mortality rates obtained from the local health authorities were compared between this cohort and neighboring areas.

Results: 451 patients were included in this study. Mortality rate was lower ( $0.67 \%$ vs. $2.17 \%, 2.22 \%, 2.23 \%$ and $2.71 \%$ in this cohort vs neighboring areas) in this cohort with early digital monitoring. All 3 deaths in this monitored cohort appeared in elderly patients who refused intensive care treatment. The hospitalization rate was $14.86 \%$ with a median of $4(6-9)$ days. Conclusion: We conclude that early remote digital monitoring and application of this system significantly reduces COVID-19 mortality. This model of outpatient care may be applied to other pandemics and is suited for use with highly infectious diseases in order to improve patient outcome and optimize resource allocation. 


\section{Case Reports}

\section{P10}

\section{Schwere Bleivergiftung durch ayurvedisches Heilmittel}

\section{Scherbaum, K. Lemberg, I. Tappert' ${ }^{1}$ T. Frank', C. Hüser, R.-U. Müller,} M. Hackl, V. Burst, V. Suárez

Nephrologie, Rheumatologie, Diabetologie und Allgemeine Innere Medizin, Klinik II für Innere Medizin, Universitätsklinikum Köln, Köln; ' ${ }^{G}$ astroenterologie und Hepatologie, Diabetologie und Stoffwechselstörungen, Klinik für Innere Medizin II, St.-Katharinen-Hospital Frechen, Frechen;

Einleitung: Berufliche Bleivergiftungen sind in den Industrienationen selten geworden [1]. Allerdings kommt es regelmäßig zu teils schweren Intoxikationen, z. B. durch Drogenkonsum (Streckungsmittel) [2] oder Präparate der ayurvedischen Medizin, wo Blei gegen eine Vielzahl von Leiden eingesetzt wird [3]. Blei wird nach Aufnahme in alle Gewebe des Körpers umverteilt und führt unter anderem zu einer Hemmung von Enzymen der Hämsynthese. Schwere Vergiftungen können Nieren- und Leberversagen sowie irreversible neurologische Schädigungen auslösen.

Kasuistik: Ein 24-jähriger Mann stellte sich mit seit Wochen bestehenden krampfartigen Unterbauchschmerzen und Leistungsknick in der Notaufnahme vor. Eine zuvor erfolgte Abklärung im Rahmen eines stationären Krankenhausaufenthaltes hatte keinen richtungsweisenden Befund erbracht. Nebenbefundlich war eine hyporegenerative Anämie aufgefallen. Es bestanden keine Vorerkrankungen, abgesehen von ehemaligem Kokain- und Cannabis-Abusus. Der Patient berichtete von einem Heilmittel aus Indien, welches er seit einem Monat einnehme (2 Kapseln/d). Befunde: Reduzierter Allgemeinzustand, blasses-graues Hautkolorit, dezenter gingivaler Bleisaum. Normozytäre, normochrome Anämie (Hämoglobin 8,7 g/dl), erhöhte Transaminasen (GOT $103 \mathrm{U} / \mathrm{l}$, GPT: $132 \mathrm{U} / \mathrm{l}$, gGT $304 \mathrm{U} / \mathrm{l})$ sowie erhöhte Lipase (608 U/l). Nachweis einer Bleiintoxikation (EDTA-Blut: $700 \mathrm{mcg} / \mathrm{l}$, [4]. Wir leiteten eine Chelatortherapie ein (DMPS [DIMAVAL ${ }^{\circ}$ ], $250 \mathrm{mg}$ i.v. alle $4 \mathrm{~h}$, Oralisierung an Tag 4 für zunächst 4 Wochen). Der Bleigehalt im EDTA-Blut sank hierunter von initial $700 \mathrm{~g} / \mathrm{l}$ auf $570 \mathrm{~g} / \mathrm{l}$ nach 4 Tagen. Die Beschwerden waren deutlich rückläufig, der Patient befindet sich in regelmäßiger Kontrolle.

Schlussfolgerung: Die Diagnose einer Bleivergiftung ist aufgrund der unspezifischen Symptome erschwert. Bei anamnestischen Hinweisen sollte daher niedrigschwellig auf Blei im Blut getestet und bei positivem Nachweis rasch mit einer Chelatortherapie begonnen werden.

\section{Literatur}

1. Centers of Disease Control (CDC) (2013) MMWR Morb Mortal Wkly Rep 62(47):967-971

2. Busse FP, Fiedler GM (2008) Bleiintoxikationen durch gestrecktes Marihuana in Leipzig. Dtsch Arztebl 105(44):757-62

3. Gunturu KS, Nagarajan P (2011) Ayurvedic herbal medicine and lead poisoning. J Hematol Oncol 4:51

4. WHO-Report TRS 896-JECFA 53/81: provisional tolerable weekly intake (PTWI) $0.025 \mathrm{mg} / \mathrm{kg} \mathrm{KG}$

\section{P11}

\section{va-ECMO bei Sepsis assoziierte Purpura fulminans}

\section{T. S. Meisel, C. Link', M. Geibel, T. Volk, A. Meiser}

Klinik für Anästhesiologie, Intensivmedizin und Schmerztherapie, Universitätsklinik Homburg, Universität des Saarlandes, Homburg/ Saar; ${ }^{1}$ Intensivmedizin, Klinik für Anästhesiologie, Intensivmedizin und Schmerztherapie, Universitätsklinikum des Saarlandes, Homburg/Saar;

Hintergrund: Die Indikation der ECMO Therapie in der Sepsis wird kritisch diskutiert. Mit dem Rücken zur Wand mussten wir uns die Frage stellen. ob eine ECMO Therapie bei einer fulminat verlaufenden Sepsis noch eine sinnvolle Therapieoption darstellt.

Methode: In der Fallvorstellung berichten wir von einer 57-jährigen Patientin mit linksseitigen Unterbauchbeschwerden, laborchemischen Infekt- zeichen und Fieber. Die Computertomographie zeigte einen prävesikalen Harnleiterstein als Ursache einer Pyelonephritis und Ureteritis. Am Aufnahmeabend erfolgte die Einlage eine Mono-J-Schiene. Innerhalb einer Stunde verschlechterte sich der Zustand massiv. Die Patientin entwickelte eine fulminante Sepsis mit Ausbildung einer Purpura. Kurzfristig war die Patientin reanimationspflichtig bei Kammerflimmern. Als Ultima ratio implantierten wir eine va-ECMO und konnten die Patientin damit stabilisieren. Die initial kalkulierte antiinfektive Therapie mit Meropenem erwies sich als sensibel für die im Punktat der Niere nachgewiesenen Keime (Proteus mirabilis, Klebsiella pneumoniae).

Ergebnisse: Die Patientin stabilisierte sich rasch unter antiinfektiver Therapie, Nierenersatztherapie und va-ECMO, und konnte nach 5 Tagen von der ECMO entwöhnt werden. Flächige retikuläre Suffusionen und akrale Nekrosen, teils konfluierend mit Spannungsblasen waren Eintrittspforten für die Entwicklung einer Candidämie mit Augenbeteiligung. Diese erforderte neben der Behandlung mit Caspofungin eine Pars Plana Vitrektomie. Die Nierenfunktion verbesserte sich und die Nierenersatztherapie konnte beendet werden. Die Patientin wurde von der Beatmung entwöhnt und auf einer Normalstation weiter behandelt. Die akralen Nekrosen der Finger und beider Füße machten leider nach Mumifizierung Amputationen notwendig.

Zusammenfassung: Die Sepsis fulminans ist das Musterbeispiel für die aktuelle Sepsisdefinition im Sinne einer lebensbedrohlichen Organdysfunktion, hervorgerufen durch eine inadäquate Wirtsantwort auf eine Infektion. Die Purpura fulminans ist eine seltene akut lebensbedrohliche Erkrankung mit hoher Mortalität ( $>50 \%$ ), die durch disseminierte Thrombosen in der Mikrozirkulation, kutane Hämorrhagien mit fortschreitender Nekrotisierung sowie multiplem Organversagen gekennzeichnet ist. Der Zusammenbruch des intrinsischen Antikoagulationssystems beruht wesentlich auf einem Protein-C-Mangel. Die supportive Anlage eine va ECMO bei Sepsis mit septischer Kardiomyopathie sollte als Ultima ratio in Betracht gezogen werden.

\section{P12}

\section{EBV assoziierte Hämophagozytzische Lymphohistiozytose}

D. O. Wennmann, H. Pavenstädt', P. Kümpers

Sektion Interdisziplinäre Notaufnahme, Medizinische Klinik D, Westfälische Wilhelms-Universität Münster, Münster; ${ }^{1}$ Allg. Innere Medizin und Notaufnahme sowie Nieren- und Hochdruckkrankheiten und Rheumatologie, Medizinische Klinik D, Westfälische Wilhelms-Universität Münster, Münster

Hintergrund: Das Krankheitsbild der Hämophagozytischen Lymphohistiozytose (HLH) stellt eine differenzialdiagnostische Herausforderung bezüglich der Diagnose und der Ursachenabklärung dar. Die HLH gehöht zur Gruppe der hyperferritinämischen Hyperinflammationssyndrome. Das Krankheitsbild ähnelt häufig einer Sepsis und ist durch Fieber, Hepatosplenomegalie und Bi- bzw. Panzytopenie gekennzeichnet. Jedes Organ kann im Verlauf betroffen sein. Neben Lymphomen, Leukämien und Autoimmunerkrankungen könne auch Infektionen, insbesondere auch eine Epstein-Barr Virus (EBV) Infektion ursächlich sein. EBV ist ein weit verbreitetes Herpes Virus, dessen Infektion häufig inapparent verläuft.

Methode: Ein 22-jähriger männlicher Patient stellt sich mit seit einer Woche bestehenden Kopfschmerzen und Fieber in der Notaufnahme vor. Die Vitalzeichen sind bis auf eine Sinustachykardie unauffällig. Körperlicher Untersuchungsbefund und Sonographie bleiben ohne pathologischen Befund. Im Aufnahmelabor findet sich eine Thrombopenie, eine leichte Hyponatriämie, eine Erhöhung des Serum-Kreatininwertes auf 1,5 mg/dl, ein erhöhtes CRP 1,4 mg/dl ( $<0,5 \mathrm{mg} / \mathrm{dl})$ sowie einer Erhöhung des indirekten Bilirubins auf $2 \mathrm{mg} / \mathrm{dl}$. Mit dem Verdacht auf eine unklare Infektion oder Systemerkrankung mit Nierenbeteiligung erfolgt die Aufnahme auf die nephrologische Normalstation.

Ergebnisse: Das nephrologische workup bleibt ohne weigweisenden Befund. Trotz erhöhten Procalcitonins bleiben Blutkulturen negativ. Die Serologie für die häufigsten bakteriellen und viralen Erreger bleibt negativ. Die Serologie für EBV zeigt das Bild einer abgelaufenen EBV Infektion. 
Ferritin und Triglyzeride zeigen sich nur leichtgradig erhöht. Der Patient entwickelt eine ausgeprägte Panzytopenie. Eine Knochenmarkhistologie bleibt unauffällig. Die nachgeforderte EBV-PCR in Blut ist positiv. Es wird die Diagnose einer EBV assoziierten HLH gestellt und eine immunsuppressive Therapie eingeleitet. Trotzdem verstirbt der Patient kurze Zeit später im Multiorganversagen auf der Intensivstation. Eine Obduktion sichert die Diagnose einer HLH postmortal.

Zusammenfassung: Die frühe Diagnosestellung ist für die Prognose der Patienten entscheidend. Das Krankheitsbild kann einer Sepsis ähnlich sein und die diagnostischen (HLH-) Kriterien müssen insbesondere initial nicht in voller Ausprägung vorliegen. Verdachtsfälle sollte stringent abgeklärt werden und serologische Untersuchungen durch PCRs ergänzt werden.

\section{P13}

Erste Anwendung eines in Deutschland neuen Verfahrens mit katheterbasierter mechanischer Thrombektomie im Rahmen einer multimodalen Therapie einer fulminanten Lungenembolie

M. Ritzka, S. Schweiger, K. Debl ' , A. Philipp ' , A. Zweckerl, J. Spörrer,

J. Wörrlein ${ }^{2}$, T. Dienemann, D. Lunz ${ }^{2}$, S. Sossalla ${ }^{1}$

Operative Intensivstation, Klinik für Chirurgie, Universitätsklinikum Regensburg, Regensburg; ${ }^{1}$ Klinik für Herzchirurgie, Universitätsklinikum Regensburg, Regensburg; ${ }^{2}$ Klinik für Anästhesiologie, Universitätsklinikum Regensburg, Regensburg;

Hintergrund: Eine sonst gesunde 55-jährige Patientin entwickelte nach einem Polytrauma und operativer Versorgung (Zwerchfellrekonstruktion, Osteosynthesen) eine fulminante zentrale Lungenembolie bei Nachweis einer Thrombose in der linken V. poplitea. Auf der Intensivstation Wechsel des Antikoagulanz bei Nachweis einer HIT II. In der Echokardiographie dilatierter rechter Ventrikel sowie bis $6 \mathrm{~cm}$ messende echoarme Struktur im Vorhof, bis in den rechten Ventrikel reichend. Entschluss zur systemischen Lyse, Blutungen im Folgenden eher mäßig.

Aufgrund kardialer Dekompensation invasive Beatmung. In den nächsten Stunden trotz medikamentöser Therapie keine Stabilisierung, sogar Reanimation notwendig, daher Implementierung einer va-ECMO über prophylaktisch vorgelegte Leistenkatheter.

Methode: Mit einem perkutanen, interventionellen Thrombektomieverfahren (Inari FlowTriever Device, Fa. Inari Medical, USA) konnte im Herzkatheterlabor über einen Zugang in der rechten V. femoralis eine $26 \mathrm{Fr}$ Schleuse eingebracht und die Pulmonalarterie sondiert werden. Über den 24 Fr Aspirationskatheter wurden Thromben direkt aspiriert. Zusätzliche Extraktion mittels FlowTriever mit im Thrombus entfaltbaren Disks (Inari medial). Kumulativ konnte so frisches, aber auch eher subakutes Material entfernt werden. Pro Aspiration wurden ca. 50-70 ml Blut abgesogen, so dass eine Transfusion notwendig war. Postinterventionell sofort Reduktion der Rechtsherzbelastung. Rechter Ventrikel basal zuvor $53 \mathrm{~mm}$, dann $36 \mathrm{~mm}$ messend.

Ergebnisse: In der Folge rasche Entwöhnung von ECMO, Medikamenten und Respirator, kurz dialysepflichtig. 10 Tage nach der Intervention im Echo wieder normdimensionierte Ventrikel ohne Zeichen einer Rechtsherzbelastung. Die neurologisch unauffällige Patientin wurde auf Marcumar umgestellt.

Zusammenfassung: Die Therapie einer fulminanten Lungenembolie verlangt eine enge Kooperation diverser medizinischer Fachgebiete in Diagnostik und Therapie, der Intensivpflege und Kardiotechnik. Ein neuartiges interventionelles großlumiges Thrombektomieverfahren bietet die Option, die Perfusion der Pulmonalarterien akut zu optimieren, den rechten Ventrikel zu entlasten und somit auch Spätschäden zu reduzieren. Es bedarf eines sicheren Gefäßzugangs und hämodynamischer Stabilität während der Prozedur. Insbesondere Gefäßverletzungen und Blutungen sind Risiken des Verfahrens. Wir berichten über den ersten erfolgreichen Einsatz des FlowTrievers außerhalb der USA.

\section{Literatur}

1. (2019) J Am Coll Cardiol Intv 12:859-869

\section{P14}

Rezidivierende retroperitoneale Blutungen bei angeborener Afibrinogenämie: Zwischen Über- und Unterdiagnostik

\section{N. Leschowski, P. Kümpers ' ${ }^{1}$ D. Hempel ${ }^{2}$}

Medizinische Fakultät, Otto-von-Guericke-Universität Magdeburg, Magdeburg; ${ }^{1}$ Sektion Interdisziplinäre Notaufnahme, Medizinische Klinik D, Westfälische Wilhelms-Universität Münster, Münster; ${ }^{2}$ Zentrale Notaufnahme, Asklepios Klinik Wandsbek, Hamburg;

Hintergrund: Die angeborene Afibrinogenämie ist eine seltene Gerinnungsstörung mit reduzierter oder fehlender Synthese des Gerinnungsfaktors Fibrinogen, welche zu spontanen lebensbedrohlichen Blutungen führen kann.

Methode: Fallbericht: Ein 37-jähriger Patient stellte sich aufgrund eines seit drei Tagen bestehenden abdominellen „Völlegefühls“ in der Notaufnahme vor. Er berichtete von einer angeborenen Afibrinogenämie mit rezidivierenden spontanen retroperitonealen Blutungen, welche sich jeweils mit ähnlichen Beschwerden bemerkbar gemacht hätten. In der körperlichen Untersuchung zeigte sich lediglich ein leichter Druckschmerz periumbilikal bei normalen Vitalzeichen. Laborchemisch bestätigte sich die bekannte Afibrinogenämie (TPZ/Quick < $5 \%$, INR 10.00, PTT $>160$ sek., Fibrinogen $<40 \mathrm{mg} / \mathrm{dl}$ ). Der Hämoglobin-Wert zeigte sich jedoch normwertig. In der fokussierten Abdomensonographie waren verplumpte Nieren ohne Nachweis freier Flüssigkeit darstellbar. Aufgrund der massiv kompromittierten Gerinnung und der mehrfachen stattgehabten retroperitonealen Blutungen erfolgte eine Computertomografie mit Kontrastmittel, welche keine Anzeichen eines neu aufgetretenen retroperitonealen Hämatoms zeigte.

Ergebnisse: Anhand dieses Falls wird die Bedeutung des „shared decision making "zur Vermeidung einer möglicherweise gefährdenden Über- oder Unterdiagnostik diskutiert. Sollte bei klinisch und laborchemisch fehlenden Blutungszeichen zum Nachweis bzw. Ausschluss einer akuten retroperitonealen Blutung bei bestehender Koagulopathie eine kontrastmittelgestützte Computertomographie des Abdomens (KM-CT) erfolgen? Oder kann auf diese verzichtet werden, um die bereits hohe kumulative Strahlenbelastung des Patienten zu verringern? Im Falle einer stabilen Kreislauffunktion und milder Symptomatik können im Sinne eines „shared decision making" mit dem Patienten die verfügbaren diagnostischen Optionen mit dem jeweiligen Nutzen und Schaden abgewogen werden. So könnten als Alternativen zur KM-CT eine engmaschige klinische Überwachung mit regelmäßigen Hämoglobinwert-Kontrollen oder eine dringliche Magnetresonanztomographie (nicht immer verfügbar) diskutiert werden.

Zusammenfassung: Der Fall verdeutlicht den schmalen Grat zwischen Über- und Unterdiagnostik beim klinischen Management eines Patienten mit milder Symptomatik aber relevanter Blutungsneigung am Beispiel der angeborenen Afibrinogenämie.

\section{P15}

Casivirimab/Imdevimab bei schwerer COVID-19 Pneumonie unter Rituximab-Induktionstherapie bei ANCA-assoziierter Vaskulitis

J. Herold, P. Zwickel', F. Geis', N. van Drongelen', M. Rosenberg', S. Büttner' Gastroenterologie, Medizinische Klinik III, Klinikum AschaffenburgAlzenau gGmbH, Aschaffenburg; ${ }^{1}$ Kardiologie, Pneumologie, Nephrologie, Internistische Intensivmedizin, Medizinische Klinik I, Klinikum Aschaffenburg-Alzenau gGmbH, Aschaffenburg;

Hintergrund: Die ANCA-assoziierte Vaskulitis ist eine Gruppe von Vaskulitiden, die die Atemwege betreffen können und unbehandelt mit einer hohen Mortalität assoziiert ist. Der gegen CD20 gerichtete monoklonale Antikörper Rituximab und Steoide sind Eckpfeiler der Induktiosntherapie. Die Sicherheit von Rituximab im Zusammenhang mit COVID-19 ist unbekannt. So kann es über die B-Zell-Depletion und eine mögliche verminderte virale Clearance zu einer potenziell schweren Covid-19 Pneumonie kommen

Methode: Wir berichten den erfolgreichen Einsatz von Casivirimab/Imdevimab (REGN-COV2) als Off-Label-Indikation bei einer schweren noso- 
komialen COVID-19 Pneumonie während der Induktionstherapie eines pulmorenalen Syndrom mit Rituximab.

Ergebnisse: Die 81-jährge Patientin wurde mit hydropischer Dekompensation, akutem Nierenversagen und CT-morphologisch milchglasartigen Eintrübungen mit kleinen Konsolidierungen stationär aufgenommen. Sowohl der SARS-CoV-2-Antigentest als auch die PCR waren initial negativ. In der Nierenbiopsie zeigte sich eine diffus extrakapillär proliferierende und nekrotisierende Glomerulonephritis mit frischen Halbmonden und serologisch konnten pANCA nachgewiesen werden, so dass die Diagnose eines pulmorenalen Syndroms bei Erstdiagnose einer mikroskopischen Polyangiitis gestellt wurde. Unter der eingeleiteten Induktionstherapie mit Steroid, Plasmapherese und Rituximab kam es zu einer nosokomialen SARS-CoV2-Infektion. Es entwickelte sich rasch eine progrediente respiratorische Partialinsuffizienz und eine es wurde eine NIV-Beatmung im Wechsel mit einer nasalen High-Flow-O2-Therapie notwendig. Bei zunehmend angestrengter respiratorischer Situation (FiO2: $80 \%$ ) entschlossen wir uns bei gesicherter Covid-19-Infektion unter B-Zell-zu einer off-LabelTherapie mit Casivirimab und Imdevimab (REGN-COV2). Unter der experimentellen Antikörpertherapie sowie den supportiven Maßnahmenbesserte sich der klinische Zustand langsam, sodass die NIV und High-Flow Behandlung im Verlauf beendet werden konnte und die Patientin wieder auf Normalstation und im Verlauf ins Pflegeheim verlegt werden konnte. Zusammenfassung: Die Induktionstherapie eines pulmorenalen Syndroms bei p-ANCA assoziierter Vaskulits mit nekrotisierender Glomerulonephritis und alveolären Hämorrhagien mit Steroid und B-Zell-Depletion ist besonders in Pandemiezeiten nicht unproblematisch. Die Gabe von Casivirimab und Imdevimab kann, wie unser Fallbericht zeigt, bei schwerer Covid-19-Pneumonie unter Rituximab eine therapeutische Option sein.

\section{P16}

\section{Haemophagocytic lymphohistiocytosis in COVID-19: Case reports of a stepwise approach}

S. Schnaubelt, D. Tihanyi', J. Oppenauer, F. Eibensteiner, H. Domanovits Universitätsklinik für Notfallmedizin, Medizinische Universität Wien, Wien/A; 'Abt. für Atemwegs- und Lungenerkrankungen, Wiener Gesundheitsverbund, Klinik Penzing, Wien/A;

Objective: The immunologic syndrome induced by severe acute coronavirus disease 2019 (COVID-19) is yet not fully understood. Typical patterns of clinical and laboratory features match secondary haemophagocytic lym-

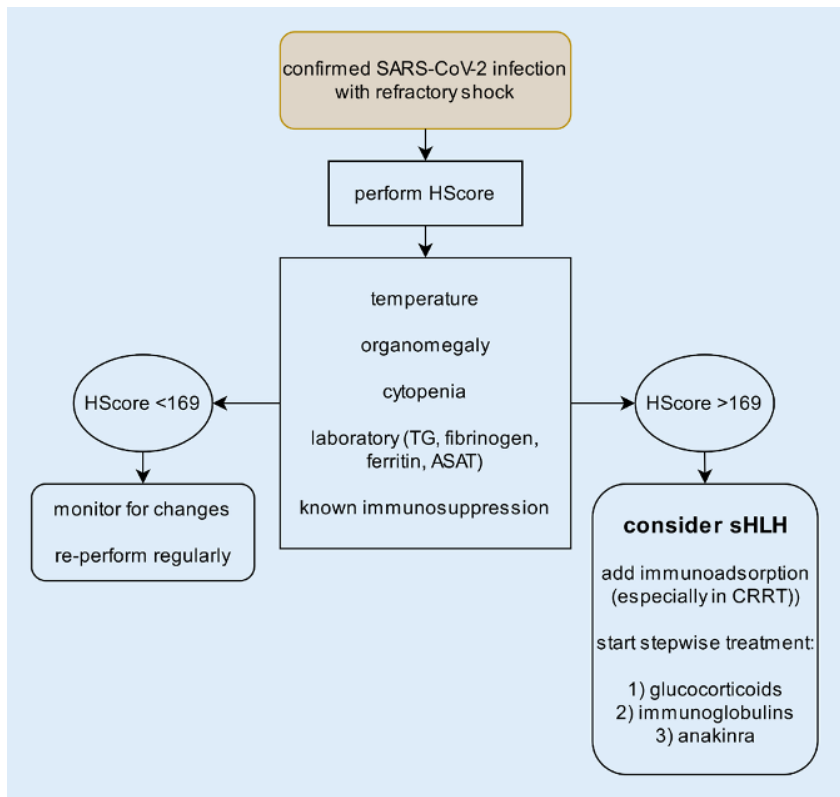

Fig. 1 | P16 phohistiocytosis (sHLH). However, the optimal approach to COVID-19 patients testing positive for sHLH is still unclear.

Method: Three patients with COVID-19 are reviewed. All showed hyperinflammation and cytokine storm, necessitating intensive care treatment including mechanical ventilation.

Results: Secondary haemophagocytic lymphohistiocytosis due to severe COVID-19 was diagnosed via HScore.

A treatment regimen of methylprednisolone, pentaglobin, and anakinra was developed and administered.

One patient survived the ICU stay. Two other patients, in whom sHLH was diagnosed too late, deceased.

Conclusion: A routine screening of COVID-19 patients for secondary $\mathrm{HLH}$ by using the HScore is feasible; especially those patients deteriorating clinically with no sufficient response to shock management might be at particular high risk. A stepwise therapeutic approach comprising corticosteroids, immunoglobulins and anakinra, accompanied by immunoadsorption, may dampen cytokine storm effects, and potentially reduce mortality (• Fig. 1).

\section{Allgemeine Intensivmedizin}

\section{P17}

36 Grad und es wird noch kälter: Messung der

Körperkerntemperatur mittels Doppelsensortechnologie während des zielgerichtetem Temperaturmanagements bei Patienten nach Kreislaufstillstand und „return-of-spontaneous circulation" im Vergleich zum Ösophagusthermometer

D. Janke, N. Kagelmann, C. Storm' ', M. A. Maggioni, H.-C. Gunga, O. Opatz

Zentrum für Weltraummedizin, Institut für Physiologie, Charité -

Universitätsmedizin Berlin, Berlin; 'Medizinische Klinik mit Schwerpunkt Internistische Intensivmedizin und Nephrologie, Campus Virchow-Klinikum, Charité - Universitätsmedizin Berlin, Berlin;

Hintergrund: Die Standardmethode zur exakten Bestimmung der Körperkerntemperatur in der Klinik ist die ösophageale oder pharyngeale Messung. Diese findet z. B. beim gezielten Temperaturmanagement (TTM) nach Herz-Kreislauf-Stillstand ihre Anwendung, ist jedoch invasiv, wird schlecht vom wachen Patienten toleriert und führt häufig zu Blutungen im Nasopharynx. Ein nicht-invasiver Einweg-Temperatursensor, der nach

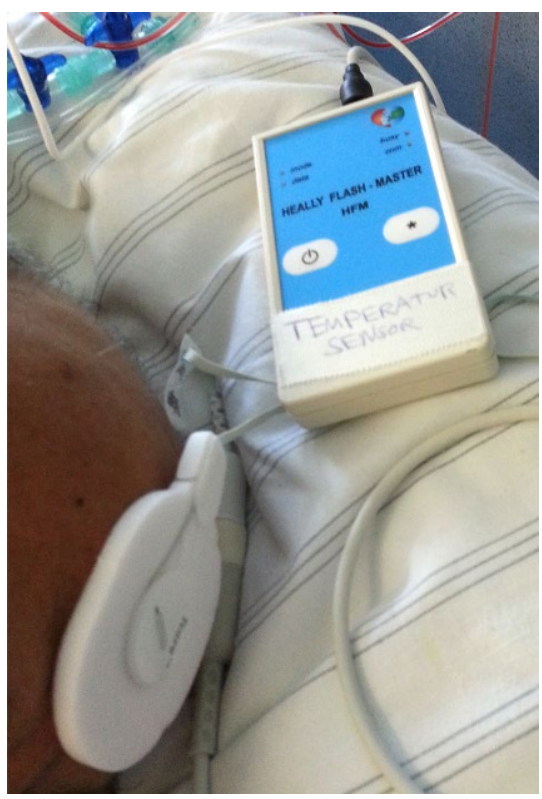

Abb. $1 \mid \mathrm{P} 174$ 


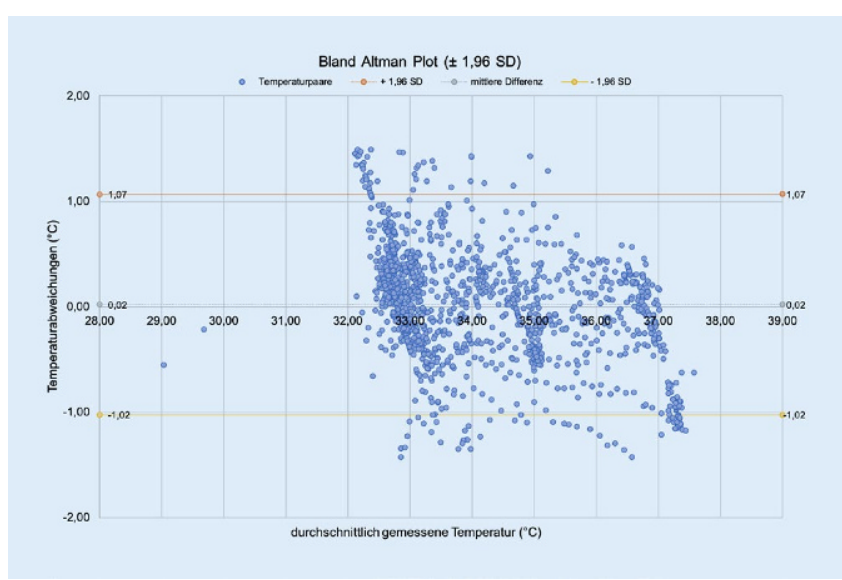

Abb. $2 \mid$ P17 $\Delta$

dem Wärmestromprinzip funktioniert, könnte hier eine gute Alternative bieten. Wir testeten eben diesen Sensor gegen den klassischen ösophagealen Sensor beim TTM nach „return-of-spontaneous-circulation“ (ROSC). Methode: Ein nicht-invasiver Einweg-Temperatursensor, welcher nach dem Wärmeflussprinzip funktioniert, wurde während ROSC unter TTM auf der Stirn befestigt (• Abb. 1).

Die aufgezeichneten Temperaturen wurden mit der etablierten Messmethode eines Ösophagusthermometers verglichen. Ein gepaarter t-Test wurde durchgeführt, um Unterschiede zwischen den Methoden zu untersuchen. Bland-Altman-Plot und der Intraklassenkorrelationskoeffizient (ICC) wurden verwendet, um die Übereinstimmung zwischen den Methoden, sowie die Zuverlässigkeit des Doppelsensors zu bewerten.

Ergebnisse: Von November 2015 bis Januar 2017 wurden Temperaturdaten von 25 Patienten mit ROSC über einen Zeitraum von 48 Stunden während TTM aufgezeichnet und nach zeitlicher Paarung miteinander verglichen. Der t-Test zeigte keinen signifikanten Unterschied zwischen den beiden Messmethoden ( $\mathrm{t}=1,47, p=0,14, n=1319$; $\bullet$ Abb. 2 ).

Der Bland-Altman-Plot zeigte eine mittlere Abweichung von $0,02{ }^{\circ} \mathrm{C}(95 \%$ Konfidenzintervall (CI) $0,00-0,04$ ) und $95 \%$ Übereinstimmungsgrenzen von $-1,023^{\circ} \mathrm{C}$ und $1,066^{\circ} \mathrm{C}$. Der Intraklassenkorrelationskoeffizient lag bei 0,94 (95\% CI 0,93-0,95).

Zusammenfassung: Die Messung der Körpertemperatur mit einem nichtinvasiven Einweg-Doppelsensor zeigt eine sehr gute Zuverlässigkeit während TTM nach überlebtem Herzstillstand. Eine Temperaturüberwachung mit dem Sensor ist somit bei diesen Fällen möglich. Auch die Steuerung interner oder externer Kühlmaschinen wäre denkbar und sollte noch in einem größeren Patientenkollektiv überprüft werden.

\section{P18 \\ Die Frühmobilisation von Intensivpatient_Innen mittels robotischem System - Ein Scoping Review}

A. C. Klamt, L. Schmidbauer, A. Warmbein ', I. Rathgeber ' , U. Fischer ${ }^{2}$, I. Eberl Professur für Pflegewissenschaften, Katholische Universität EichstättIngolstadt, Eichstätt; ${ }^{1}$ Klinikum der Ludwigs-Maximilians-Universität München, München; ${ }^{2}$ Institut für medizinische Informationsverarbeitung, Biometrie und Epidemiologie, Ludwig-Maximilians-Universität München, München;

Hintergrund: Die Mobilisation von Intensivpatient_Innen wird aufgrund von verschiedenen Faktoren oft sehr spät durchgeführt [1]. Studien zeigen aber, dass sie, insbesondere wenn sie früh beginnt, einen positiven Effekt auf Heilungsprozess und Rehabilitation von Schwerstkranken haben kann [2]. Robotische Systeme können dabei helfen, die Frühmobilisation im Intensivstationsalltag realisierbarer zu machen. Dieses Scoping Review gibt einen Überblick über bisherige Forschungsaktivität zur robotischen Frühmobilisation von Intensivpatient_Innen. Folgende Fragestellungen lagen zugrunde: Wie wird Frühmobilisation mittels robotischem System auf Intensivstationen durchgeführt? Welchen Effekt hat die Frühmobilisation mittels robotischem System auf das Patient_Innenoutcome? Methode: Es wurde eine systematische Literaturrecherche gemäß PRISMA Extension for Scoping Reviews [3] und der Empfehlung des JBI-Manuals [4] durchgeführt. Mittels definierter Suchstrings erfolgte zwischen Mai und Juli 2020 eine Recherche in den Datenbanken MEDLINE (via PubMed und Web of Science), CINAHL, Cochrane Library, Embase, IEEE Xplore, Scopus und WTI. Eine Randsuche erfolgte über ResearchGate und GoogleScholar. Eingeschlossen wurden Artikel die Rehabilitations-, (Früh)Mobilisations- und Transferrobotik mit dem Ziel einer Aktivierung beinhalteten.

Ergebnisse: Es konnten 14 Artikel (4 Text and Opinions, 1 Fallstudie, 2 Fall-Kontroll Studien, 1 Quasi-experimentelle Interventionsstudie, 1 Querschnittstudie und 5 RCTs) in das Review eingeschlossen werden. Die robotische Frühmobilisation von Intensivpatient_Innen erfolgt bisher überwiegend mit elektronischen Bettfahrrädern. Weiterhin werden elektronische Laufbänder oder Kipptische eingesetzt, die aber immer mit einem Transfer der Patient_Innen auf das Gerät verbunden sind. Das birgt ein Sicherheitsrisiko für die Beteiligten. Die Anwendung von Frühmobilisationssystemen hat einen positiven Effekt auf das Patient_Innenoutcome, der sich bspw. in der Stabilität von Hämodynamik und Respiration sowie in der Stärkung von Kraft und der Verbesserung der körperlichen Funktion zeigt [5-7].

Zusammenfassung: Die Ergebnisse zeigen, dass weitere Analysen zum Einsatz von robotischen Frühmobilisationssystemen nötig sind. Vor allem der Personalaufwand ist mit den aktuell eingesetzten Systemen noch sehr hoch. Klar wird auch, dass konventionelle Frühmobilisation durch robotische Systeme sinnvoll ergänzt aber nicht ersetzt werden kann.

\section{Literatur}

1. Rai S, Anthony L, Needham DM, Georgousopoulou EN, Sudheer B, Brown R, Mitchell I, van Haren F (2020) Barriers to rehabilitation after critical illness: a survey of multidisciplinary healthcare professionals caring for ICU survivors in an acute care hospital. Australian Critical Care 33(3):264-271. https://doi.org/10.1016/j.aucc. 2019.05.006

2. Waldauf P, Jiroutková K, Krajcová A, Puthucheary Z, Duka F (2020) Effects of Rehabilitation Interventions on Clinical Outcomes in Critically III Patients: Systematic Review and Meta-Analysis of Randomized Controlled Trials. CRIT CARE MED 48(7):1055-1065. https://doi.org/10.1097/CCM.0000000000004382

3. Tricco AC, Lillie E, Zarin W, O'Brien KK, Colquhoun H, Levac D, Moher D, Peters MDJ, Horsley T, Weeks L, Hempel S, Akl EA, Chang C, McGowan J, Stewart L, Hartling L, Aldcroft A, Wilson MG, Garritty C, Lewin S, Godfrey CM, Macdonald MT, Langlois EV, Soares-Weiser K, Moriarty J, Clifford T, Tunçalp Ö, Straus SE (2018) PRISMA Extension for Scoping Reviews (PRISMA-ScR): Checklist and Explanation. Ann Intern Med 169(7):467-473. https://doi.org/10.7326/M18-0850

4. The Joanna Briggs Institute (JBI) (2015) Methodology for JBI Scoping Reviews. https://joannabriggs.org/assets/docs/sumari/Reviewers-Manual Methodologyfor-JBI-Scoping-Reviews_2015_v2.pdf.

5. Frazzitta G, Zivi I, Valsecchi R, Bonini S, Maffia S, Molatore K, Sebastianelli L, Zarucchi A, Matteri D, Ercoli G, Maestri R, Saltuari L (2016) Effectiveness of a Very Early Stepping Verticalization Protocol in Severe Acquired Brain Injured Patients: A Randomized Pilot Study in ICU. PLoS ONE 11(7). https://doi.org/10.1371/journal. pone. 0158030

6. Parry SM, Berney S, Warrillow S, El-Ansary D, Bryant AL, Hart N, Puthucheary Z, Koopman R, Denehy L (2014) Functional electrical stimulation with cycling in the critically ill: a pilot case-matched control study. J Crit Care 29(4):695-e1

7. Parry SM, Berney S, Koopman R, Bryant A, El-Ansary D, Puthucheary Z, Hart N, Warrillow S, Denehy L (2012) Early rehabilitation in critical care (eRiCC): functional electrical stimulation with cycling protocol for a randomised controlled trial. BMJ Open 2(5) 


\section{P19}

\section{Verlauf von Laktat, pH und Base Excess zur Prädiktion der Mortalität bei internistischen Intensivpatienten}

\section{A. Schork, K. Moll, M. Haap ' , R. Riessen ' , R. Wagner ${ }^{2}$}

Sektion Nieren- und Hochdruckkrankheiten, Universitätsklinikum, Medizinische Klinik IV, Eberhard Karls Universität Tübingen, Tübingen; Departement für Innere Medizin, Intensivstation, Universitätsklinikum, Eberhard Karls Universität Tübingen, Tübingen; ${ }^{2}$ Abteilung für Endokrinologie und Diabetologie, Angiologie, Nephrologie und Klinische Chemie, Universitätsklinikum, Eberhard Karls Universität Tübingen, Tübingen;

Hintergrund: Eine Studie an herzchirurgischen Patienten hatte Hinweise ergeben, dass der Base Excess (BE) dem Laktatspiegel möglicherweise als Prognoseparameter bei Intensivpatienten überlegen sein könnte. Diese Hypothese sollte in dieser Studie an einem Kollektiv internistischer Intensivpatienten überprüft werden.

Methode: Laktat, $\mathrm{pH}$ und Base Excess bei Aufnahme auf die ICU und nach $24 \pm 4$ Stunden sowie die jeweils maximalen/minimalen Werte in den ersten 24 Stunden und in 24-48 Stunden nach der Aufnahme wurden von allen Patienten, die auf die internistische Intensivstation der Universitätsklinik Tübingen von Januar 2016 bis Dezember 2018 aufgenommen wurden, gesammelt und als Prädiktoren für die Mortalität während des Krankenhausaufenthaltes untersucht.

Ergebnisse: Die Mortalität in der Kohorte mit 4067 Patienten war $22 \%$ und korrelierte signifikant mit allen untersuchten Parametern. Die stärksten Prädiktoren in der ROC-Analyse waren das Laktat-Maximum in den ersten $24 \mathrm{~h}$ (AUROC 0.74, Cut off $2.7 \mathrm{mmol} / \mathrm{L}$, Hazard Ratio der Risikogruppe mit einem Wert größer dem Cut off 3.20) und das pH-Minimum in den ersten $24 \mathrm{~h}$ (AUROC 0.71, Cut off 7.31, Hazard Ratio der Risikogruppe 2.94). Die Kaplan-Meier-Kurven für Patientengruppen aufgeteilt nach diesen Cut offs zeigten eine frühe und klare Separation. Die Hazard Ratio pro Erhöhung um eine Standardabweichung war am höchsten für das Laktat-Maximum (HR 1.65), BE-Minimum (HR 1.56) und pH-Minimum (HR 0.75) jeweils in den ersten $24 \mathrm{~h}$. In der multivariablen logistischen Regression waren Alter, pH-Minimum in den ersten $24 \mathrm{~h}, \mathrm{pH} 24 \mathrm{~h}$ nach Aufnahme, Laktat-Maximum in den ersten $24 \mathrm{~h}$, Laktat-Maximum in $24-48 \mathrm{~h}, \mathrm{BE}-$ Minimum in den ersten $24 \mathrm{~h}$ und BE-Minimum in $24-48 \mathrm{~h}$ unabhängige Prädiktoren der Mortalität.

Zusammenfassung: Sowohl Laktat als auch $\mathrm{pH}$ and Base Excess sind geeignete Prädiktoren für die Mortalität bei internistischen Intensivpatienten, wobei die maximalen bzw. minimalen Werte in den ersten 24 Stunden und 24 bis 48 Stunden nach der Aufnahme auf die ICU insgesamt stärkere Prädiktoren waren als die Werte bei Aufnahme. Base Excess und pH waren dem Laktat als Prädiktoren der Mortalität bei internistischen Intensivpatienten nicht überlegen.

\section{P20}

\section{Optimierte Händedesinfektionsinfrastruktur auf der} Intensivstation -Auswirkung auf die Händedesinfektionsleistung

\section{A. N. Sharif, C. Hoffmann, F. Compton \\ Medizinische Klinik IV, Klinik für Nephrologie, Campus Benjamin Franklin, Charité - Universitätsmedizin Berlin, Berlin;}

Hintergrund: Die korrekte Durchführung der hygienischen Händedesinfektion ist für die Prävention nosokomialer Infektionen von entscheidender Bedeutung, der einfache und indikationsgerechte Zugang zu Händedesinfektionsmittel daher entsprechend wichtig. In dieser retrospektiven Studie wurde untersucht, wie sich auf einer internistischen Intensivstation die Umstellung des Händedesinfektionsmittelkonzepts von variabel aufgestelltem Desinfektionsmittel und Kitteltaschenflaschen auf an definierten Orten umfangreich fest installierte automatisierte Desinfektionsmittelspender auf die Händehygiene ausgewirkt hat.

Methode: Die Untersuchung erfolgte auf einer universitären internistischen Intensivstation mit 16 Behandlungsplätzen. Verglichen wurden Desinfektionsmittelverbrauch, Händehygiene-Compliance und nosokomina- le Infektionsraten vor (2015/2016) und nach (2018/2019) der Installation von 56 automatisierten Händedesinfektionsmittelspendern (Ingo-man ${ }^{\circ}$ smart touchless, Ophardt Hygiene-Technik), die jede Betätigung kontinuierlich erfassen. Der Desinfektionsmittelverbrauch wurde anhand der ausgelieferten Desinfektionsmittelmenge sowie der durch Spenderbetätigungen errechneten Menge ermittelt. Die Compliance wurde durch direkte Observation erfasst. Die Rate nosokomialer Infektionen wurde dem Krankenhaus-Infektions-Surveillance-System entnommen.

Ergebnisse: Im Beobachtungszeitraum wurden pro Jahr im Mittel $881 \pm 95$ Patienten behandelt, dies entsprach einer Auslastung der Intensivstation von $94,7 \% \pm 1,9 \%$. Gegenüber dem Zeitraum vor Installation der automatisierten Desinfektionsmittelspender kam es nach Umsetzung des neuen Spenderkonzepts nicht zu einer Erhöhung des Händedesinfektionsmittelverbrauchs (741 1/Jahr vs. 695 l/Jahr). Eine Zunahme der HändehygieneCompliance konnte ebenfalls nicht beobachtet werden $(63,8 \% \pm 7,7 \%$ vs. $68,7 \%$ vs. $5,1 \%, p=0,11)$. und auch beim Vergleich der nosokomialen Infektionsraten zeigten sich keine relevanten Unterschiede zwischen den Infektionsraten vor und nach der Spenderinstallation.

Zusammenfassung: In der vorliegenden Untersuchung führte die alleinige Ausstattung einer Intensivstation mit fest installierten automatisierten Händedesinfektionsmittelspendern und somit der Optimierung der Möglichkeiten für die indikationsgerechte Durchführung der Händedesinfektion nicht zu einer Verbesserung von Händedesinfektions-Compliance und Händedesinfektionsleistung.

\section{P21}

Optimale Lokationen für Desinfektionsmittelspender auf einer Intensivstation, Region of interest versus most popular regions.

\section{Hoffmann, F. Compton}

Medizinische Klinik IV, Klinik für Nephrologie, Campus Benjamin Franklin, Charité - Universitätsmedizin Berlin, Berlin;

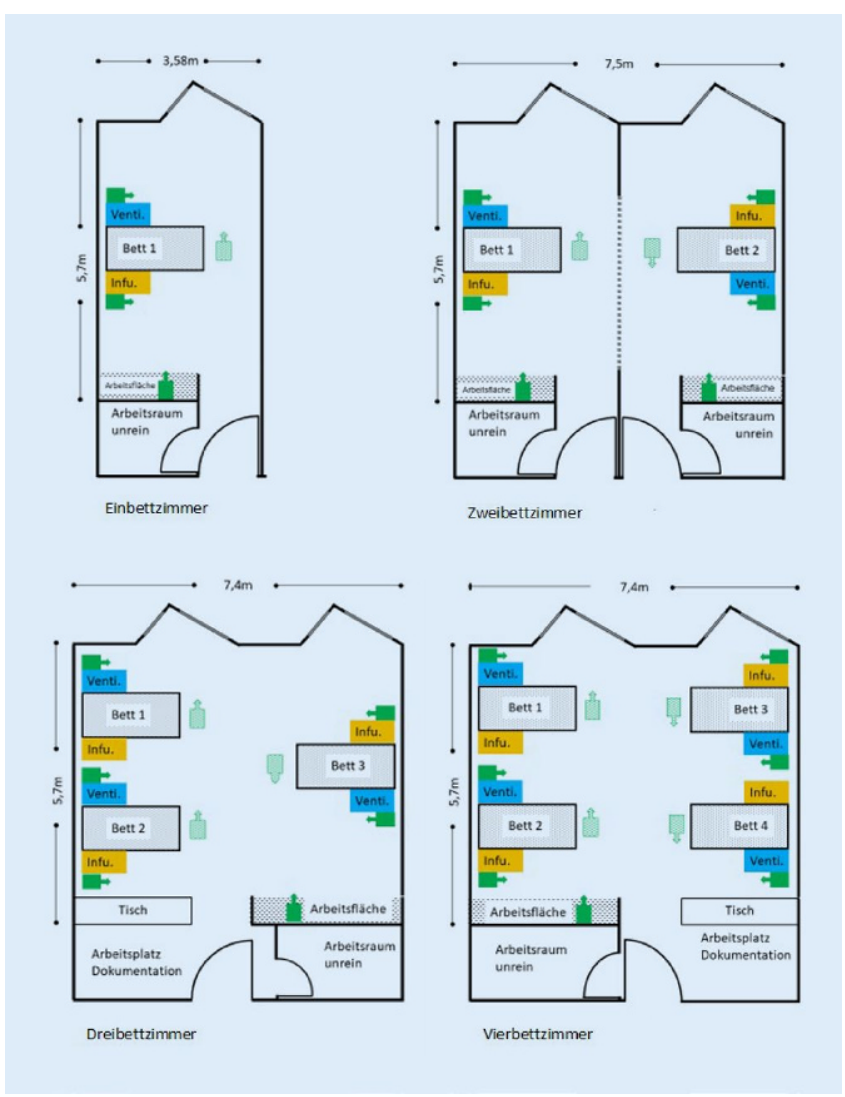

Abb. 1 |P21 A 

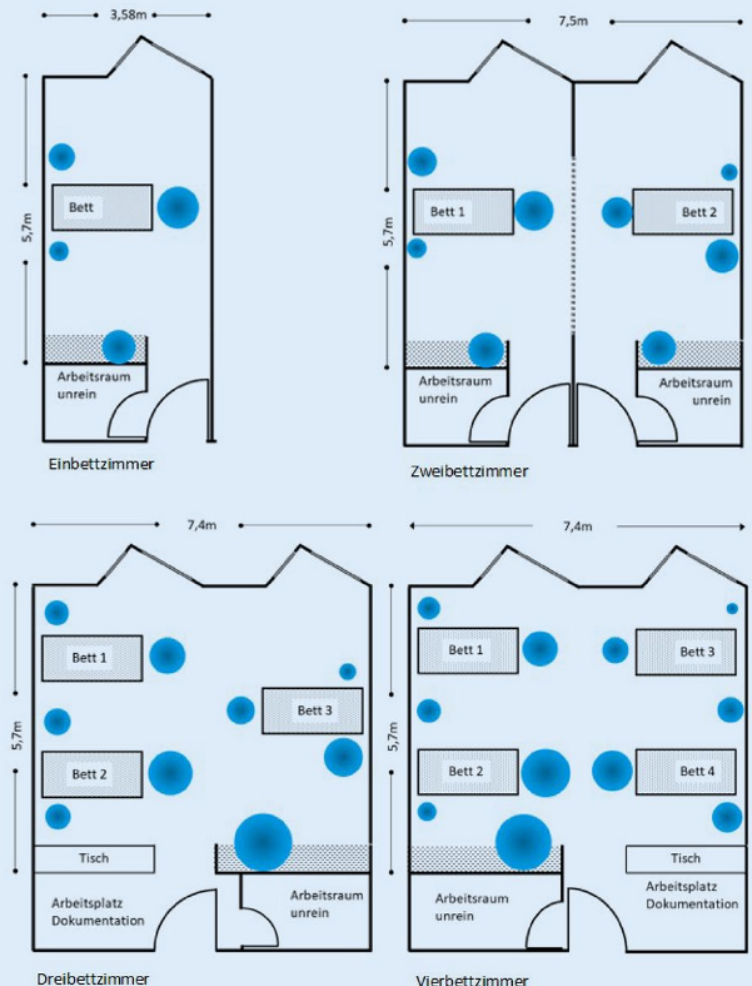

Abb. $2 \mid$ P21

Hintergrund: Die Händedesinfektionscompliance auf Intensivstationen zu verbessern wurde schon mit vielen Methoden versucht. Wir untersuchten, wie unterschiedliche Spenderlokationen frequentierte werden, wenn innerhalb des Behandlungszimmers ubiquitäre Möglichkeiten bestehen, Händedesinfektionen durchzuführen.

Methode: In einer retrospektiven Analyse überprüften wir, wo in Intensivbehandlungsräumen tatsächlich Händedesinfektionen stattfinden, wenn die Ausstattung der Station dies theoretisch überall ermöglicht.

Eine Intensivstation mit 16 Beatmungsplätzen wurde dazu mit 56 Händedesinfektionsmittelspendern ausgestattet. An allen Lokationen, die von hohem Interesse bezüglich der 5 WHO Indikationen sind, wurden Spender montiert. Dies waren die Bereiche der Infusionsgeräte und Beatmungsmaschinen, in denen die meisten aseptischen Tätigkeiten zu erwarten sind. Zusätzlich wurden Spender zentral im Raum verteilt. Die Installationsorte wurden in einer interdisziplinären Evaluationsphase über sechs Monate vor Beginn der Überprüfung optimiert. Die Verteilung der Spender innerhalb der Zimmer sind der $\bullet$ Abb. 1 zu entnehmen.

Über 3 Jahre ermittelten wir die Nutzung der Händedesinfektionsmittelspender via WLAN- Daten-übertragung der Spenderereignisse. Zusätzlich wurde die Händedesinfektionscompliance durch standardisierte Beobachtung überwacht.

Ergebnisse: 899.700 Händedesinfektionsereignisse wurden ausgewertet. Die Nutzung der Spender war extrem unterschiedlich:

- Maximal genutzter Spender: 52.637 Ereignisse/3 Jahre

- Minimal genutzter Spender: 4340 Ereignisse/3 Jahren

Verteilung: 601.940 (66,9\%) der Händedesinfektionsereignisse fanden im „Zimmerzentrum“ statt.

297.760 (33,1 \%) der Händedesinfektionsereignisse fanden in der „Zimmerperipherie" statt.

In der $\bullet$ Abb. 2 sind die Verteilungen der Händedesinfektionsereignisse graphisch dargestellt. Die Größe der blauen Kreise entspricht der Anzahl der Ereignisse.
Zusammenfassung: Anders als zu vermuten, finden zwei Drittel der Händedesinfektionsereignisse eher im „Zimmerzentrum “ (most popular) statt und nicht an den Orten, auf die aus Sicht der Krankenhaushygiene ein besonderes Augenmerk gerichtet wird („Zimmerperipher“, Region of interest). Die Vorgabe, für die Ausstattung einer Intensivstation an jeder Lokation einer Hygienetätigkeit eine Händedesinfektionsmöglichkeit zu schaffen, sollte entsprechend interpretiert werden.

\section{P22}

\section{Sex differences in ICU patients with COVID-19 in Intensive Care} Units in Tyrol (Austria) during the first wave of a global pandemic

\section{T. Mayerhöfer, S. Klein, M. Joannidis'}

Internistische Notfall- und Intensivmedizin, Department für Innere Medizin, Medizinische Universität Innsbruck, Innsbruck/A; ${ }^{1}$ Klinische Abteilung für Allgemeine Innere Medizin, Universitätsklinik für Innere Medizin, Medizinische Universität Innsbruck, Innsbruck/A;

Objective: First reports of critically ill COVID-19 patients from Wuhan indicated, that men suffer more often from critical illness and have higher mortality than women. Since than widely varying mortality rates of different cohorts all around the globe, highlighted the need of a local surveillance of Intensive Care Unit (ICU) capacities and resources. This study aimed to evaluate sex differences in the admission rate, the admission status (SAPS 3 Admission Score) and the outcomes of ICU patients in Tyrol, Austria with a SARS-CoV2 infection during the first phase (February ${ }^{1} \mathrm{st}^{-}$ July ${ }^{17}$ th) of the COVID-19 pandemic.

Method: This prospective observational cohort study included COVID-19 patients admitted to an ICU in Tyrol, Austria. Data was provided from all ICUs in Tyrol for the Tyrolean COVID-19 intensive care registry. Basic demographics, risk factors, details of the intensive care treatment and SAPS 3 Admission Scores, as well as ICU and hospital mortality have been recorded.

Results: A total of 107 patients have been admitted with COVID-19 to an ICU during February 1st and July 17th. The median age was 64 years (interquartile range [IQR] 54-74) and 77 patients were male (71.7\%). Women were significantly older (72 [IQR 55-78] vs. 63 [54-71], $p=0.034$ ) than man. While the ICU length of stay was significantly shorter in women (11 [IQR 5-21.8] vs. 21 [6-34], $p=0.038$ ), the hospital length of stay was shorter, but not statistically significant (21.5 [IQR 11.3-34.75] vs (28[IQR 16$48], p=0.070)$. There was no significant difference in the rate of invasive mechanical ventilation (IMV) $(19$ [63.3\%] vs. 53 [68.8\%], $p=0.753)$, in the median SAPS 3 Admission Score (56.50 [IQR 52.25-66.25] vs. 56.00 [IQR $47.50-63.50]$ ), or in ICU mortality (9 [30\%] vs. 14 [18.2\%], $p=0.282$ ). Conclusion: Significantly more men are admitted to the ICU because of COVID-19, which is consistent with other studies and other infectious diseases. However, if admitted to the ICU men and women had almost the same median SAPS 3 Admission Score, similar rates of mechanical ventilation and comparable mortality rates.

\section{Herz-Kreislauf}

\section{P23}

ADP-induced platelet reactivity and bleeding events in patients with acute myocardial infarction complicated by cardiogenic shock

C. Scherer, E. Lüsebrink, T. Stocker, S. Massberg, D. Sibbing, M. Orban

Medizinische Klink I, Campus Großhadern, Ludwig-Maximilians-Universität München, München;

Objective: While previous reports showed ADP-induced platelet reactivity to be an independent predictor of bleeding after PCI in stable patients, this has never been investigated in patients with cardiogenic shock. 


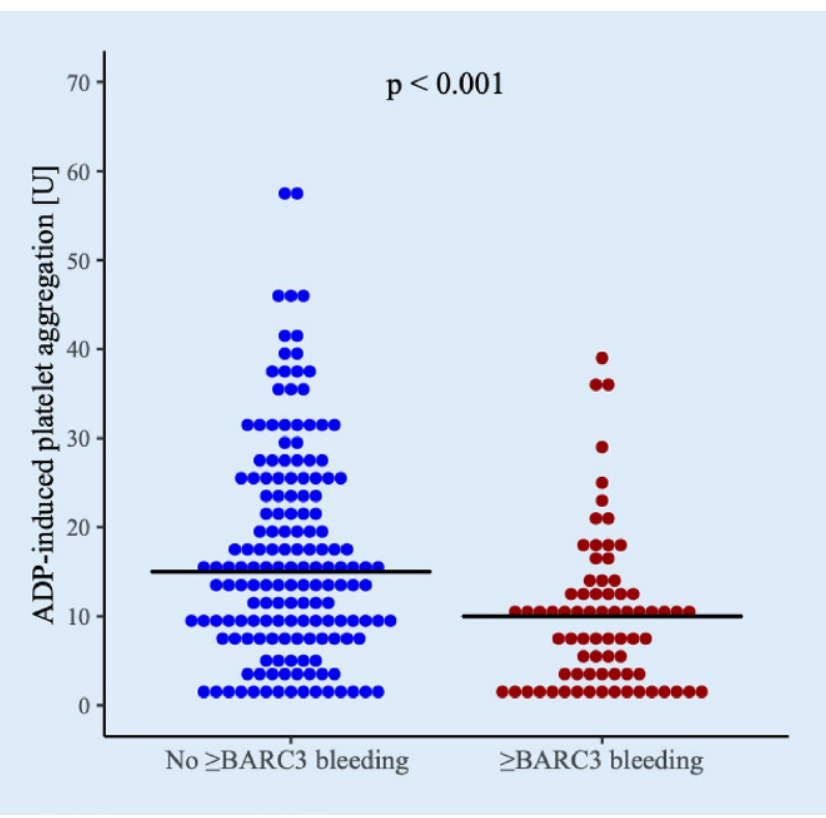

Fig. 1 | P23

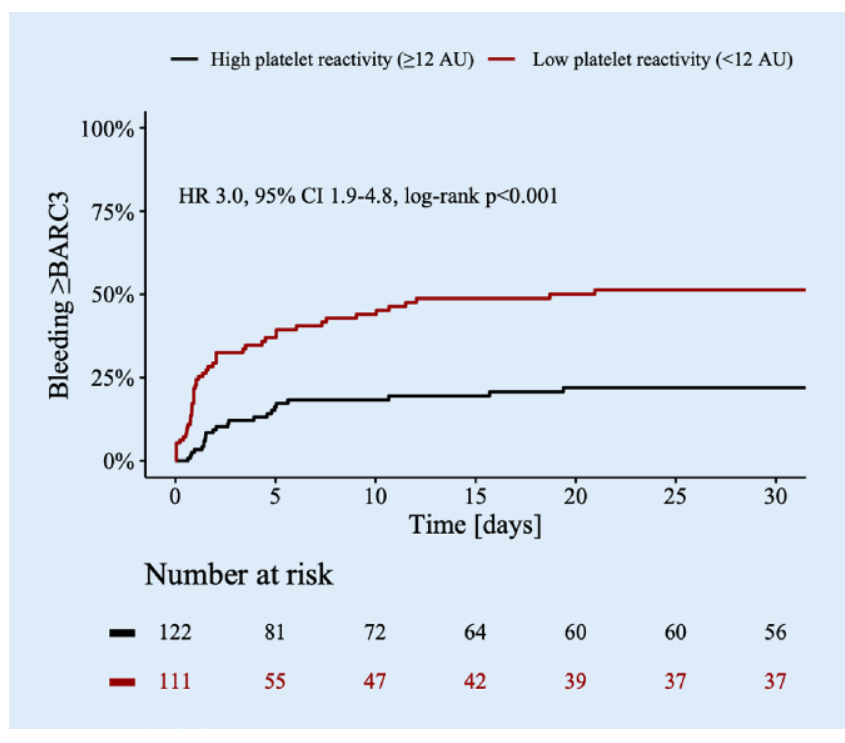

Fig. 2 | P23

Method: The association of bleeding events with respect to ADP-induced platelet aggregation was investigated in patients undergoing primary PCI for acute myocardial infarction complicated by cardiogenic shock (AMI-CS) and with available on-treatment ADP-induced platelet aggregation measurements.

Results: Out of 233 patients, 74 suffered from a severe BARC 3 or higher bleed. ADP-induced platelet aggregation was significantly lower in patients with BARC $\geq 3$ bleedings (10 AU [IQR 3-13] vs. 15 AU [IQR 9-25], $p<0.001)$. Multivariate analysis identified on-treatment ADP-induced platelet aggregation as an independent risk factor for bleeding $(\mathrm{HR}=0.968$ per AU, $95 \%$ confidence interval (CI) 0.942-0.994). An optimal cut-off value of $<12 \mathrm{AU}$ for $\mathrm{ADP}$-induced platelet aggregation to predict $\mathrm{BARC} \geq 3$ bleedings was identified via ROC analysis. Moreover, use of VA-ECMO (HR 1.972, $95 \%$ CI 1.003-3.879) or coaxial left ventricular pump (HR 2.593, $95 \%$ CI 1.509-4.455), first lactate (HR 1.093 per mmol/l, $95 \%$ CI 1.037-1.152) and thrombocyte count (HR 0.994 per G/l, $95 \%$ CI 0.990 0.998 ) were independent predictors of BARC $\geq 3$ bleedings. There was no significant difference in survival nor ischemic events between patients with low and high on-treatment platelet reactivity (• Fig. 1 and 2).

Conclusion: Lower on-treatment ADP-induced platelet aggregation was independently associated with severe bleeding events in patients with AMI-CS. The value of platelet function testing for bleeding risk prediction and guidance of anti-thrombotic treatment in cardiogenic shock warrants further investigation

\section{P24}

\section{Long-Term Clinical Outcome of Cardiogenic Shock Patients Undergoing Impella CP Treatment vs. Standard of Care}

\section{Scherer, E. Lüsebrink, T. Stocker, S. Massberg, M. Orban}

Medizinische Klink I, Campus Großhadern, Ludwig-Maximilians-Universität München, München;

Objective: The number of patients treated with the mechanical circulatory support device Impella Cardiac Power (CP) for cardiogenic shock is steadily increasing. The aim of this study was to investigate long-term survival and complications related to this modality.

Method: Patients undergoing Impella CP treatment for cardiogenic shock were retrospectively enrolled and matched with cardiogenic shock patients not treated with mechanical circulatory support between 2010 and 2020 . Data were collected from the cardiogenic shock registry of the university hospital of Munich (DRKS00015860). 70 patients with refractory cardiogenic shock without mechanical circulatory support were matched with 70 patients treated with Impella CP.

Results: At presentation, the mean age was $67 \pm 15$ years with $80 \%$ being male in the group without support and $67 \pm 14$ years $(p=0.97)$ with $76 \%$ being male $(p=0.68)$ in the group with Impella. There was no significant difference in the rate of cardiac arrest $(47 \%$ vs. $51 \%, p=0.73)$ and myocardial infarction was the predominant cause of cardiogenic shock in both groups ( $70 \%$ vs. $77 \%$ ). A total of $41 \%$ of patients without cardiocirculatory support and $54 \%$ of patients with Impella support died during the first month $(p=0.17)$. After one year, mortality rates were similar in both groups ( $55 \%$ in conventional vs. $59 \%$ in Impella CP group, $p=0.30$ ) as was mortality rate at long-term 5 -years follow-up ( $64 \%$ in conventional vs. $73 \%$ in Impella CP group, $p=0.33$ ). The rate of clinically significant bleedings during ICU stay was lower in the conventional group than in the Impella support group ( $15 \%$ vs. $43 \%, p=0.002$ ).

Conclusion: In this small observational and non-randomized analysis no difference in long-term outcome between patients treated with Impella $\mathrm{CP}$ vs. guideline directed cardiogenic shock therapy without mechanical circulatory support could be detected. Care must be taken regarding the high rate of bleeding and vascular complications when using Impella CP. Large, adequately powered studies are urgently needed to investigate the efficacy and safety of Impella CP in cardiogenic shock.

\section{P25}

\section{Radiographic assessment of lung edema (RALE) score is associated with clinical outcomes in VA-ECMO patients}

\section{Voigt, M. Mighali ', D. Manda ${ }^{1}$, P. Aurich, M. Rottorf ${ }^{2}$, O. Bruder}

Klinik für Kardiologie und Angiologie, Elisabeth-Krankenhaus Essen, Essen; ${ }^{1}$ Klinik für Akut- und Notfallmedizin, Elisabeth-Krankenhaus Essen, Essen;

${ }^{2}$ Klinik für Innere Medizin und Gastroenterologie, Elisabeth-Krankenhaus Essen, Essen;

Objective: VA-ECMO is a promising option in cardiogenic shock (CS) and as an extended resuscitation procedure (eCPR). However, the elevation in left ventricular afterload may increase myocardial wall stress, myocardial oxygen consumption and LV distention and enhances further reduction of LV contractility and pulmonary edema.

Aim of this study was to evaluate pulmonary edema based on the RALE score [1] in patients receiving VA-ECMO therapy and the prognostic value of the score on ECLS-weaning and ICU mortality. 
Method: In this retrospective study (2018-2020) we screened data of 65 patients receiving VA-ECMO and included all patients who have been treated for more than $24 \mathrm{~h}$ in our ICU. In addition to demographic and clinical data, RALE score to evaluate pulmonary edema was assessed. Descriptive statistics, intra class correlation analysis, Receiver Operator Characteristics (ROC) and logistic regression analysis were used.

Results: In total, data from 31 patients (25 eCPR, $6 \mathrm{CS}$ ) were analyzed. The median age was 59 years [IQR 49.2-67.75] and 23 (74.4\%) were male. Acute myocardial infarction was reported in $24(77.4 \%)$ and a cardiomyopathy in 7 cases $(22.6 \%)$. Initial laboratory values presenting this cohort were CKmax $3075 \mathrm{mg} / \mathrm{dl}$ [531-6078], CK-MBmax $351 \mathrm{mg} / \mathrm{dl}$ [91-542], lactate $10.1 \mathrm{mmol} / \mathrm{l}$ [5.8-13.7]. The median EF was $25 \%$ [20-35]. Overall, weaning from cardiac support was successful in 21 patients after a median of 5.0 days (2.0-5.5) and 13 patients $(41.9 \%)$ were discharged alive. The initial RALE score was 23.5 (11.2-31.8) with a good interobserver variability (0.832). During ECLS-therapy 5 pts. showed a progression, 7 pts. a regression and 19 pts. a stable radiological score. In the group with an initial RALE score $<24$, ECLS-weaning was achieved in $16 / 20$ cases $(80 \%)$ as opposed to $5 / 11(45 \%)$ with a RALE score $>24(p=0.1009)$. Mortality in the RALES group $<24$ was $9 / 20(45 \%)$ vs. $9 / 11(81 \%)$ in the RALES group $>24(p=0,1901)$.

In the ROC analysis, the area under the curve (AUC) for the initial lactate concentration was 0.84 (mortality) and 0.86(ECLS-Weaning), for the RALES score 0.80 (mortality) and 0.82 (ECLS-Weaning).

Conclusion: The RALE score can support the prognosis of ECLS-weanability and ICU mortality in patients recieving VA-ECMO therapy. It seems to be appropriate for further studies of unloading techniques (e.g. IABP, Impella) during VA-ECMO.

\section{References}

1. Warren et al (2018) Severity scoring of lung oedema on the chest radiograph is associated with clinical outcomes in ARDS. Thorax 73(9):840-846

\section{P26}

\section{Arterial stiffness in acute COVID-19 and potential associations with clinical outcome}

S. Schnaubelt, J. Oppenauer

Universitätsklinik für Notfallmedizin, Medizinische Universität Wien, Wien/A;

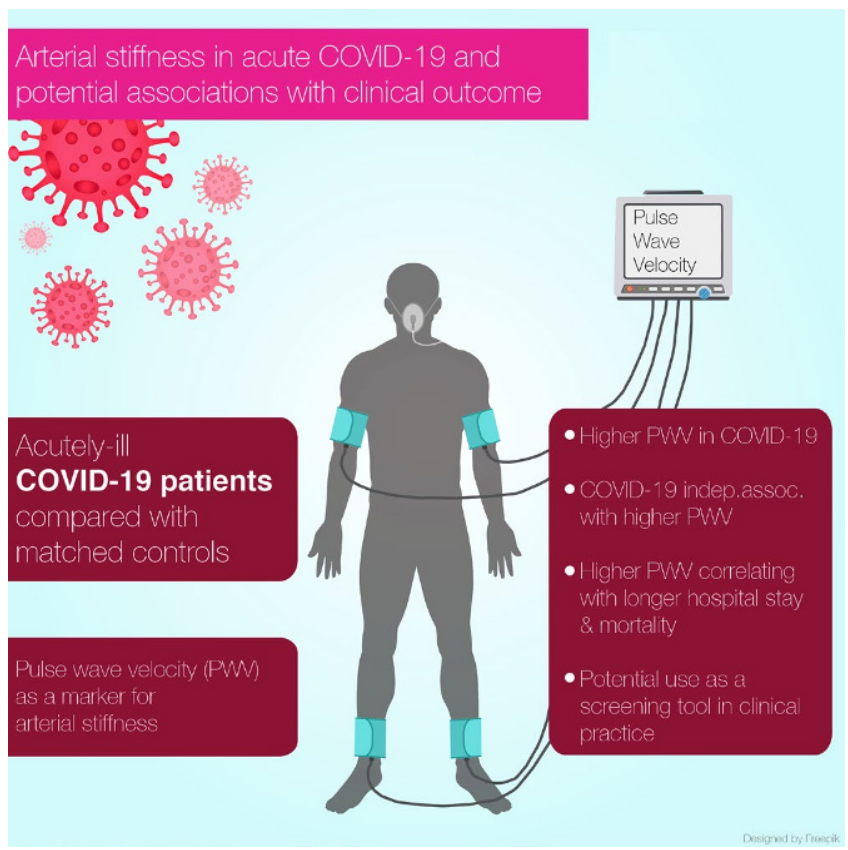

Abb. 1 |P26 $\triangle$
Objective: Coronavirus disease 2019 (COVID-19) interferes with the vascular endothelium. It is not known whether COVID-19 additionally affects arterial stiffness.

Method: This case-control study compared brachial-ankle pulse wave (baPWV)- and carotid-femoral pulse wave velocities (cfPWV) of acutelyill patients with- and without COVID-19.

Results: Twenty-two COVID-19 patients (50 \% females, 77 [67-84] years) were compared with 22 age- and sex-matched controls. In COVID-19 patients, baPWV (19.9 [18.4-21.0] vs. 16.0 [14.2-20.4], $p=0.02)$ and cfPWV (14.3 [13.4-16.0] vs. 11.0 [9.5-14.6], $p=0.01)$ were higher than in the controls. In multiple regression analysis, COVID-19 was independently associated with higher $\operatorname{cfPWV}(\beta=3.164, p=0.004)$ and baPWV $(\beta=3.532$, $p=0.003$ ). PWV values were higher in non-survivors. In survivors, PWV correlated with length of hospital stay.

Conclusion: COVID-19 appears to be related to an enhanced PWV reflecting an increase in arterial stiffness. Higher PWV might be related to an increased length of hospital stay and mortality (- Abb. 1).

\section{P27}

\section{Arterielle Gefäßsteifigkeit im akuten Koronarsyndrom als} potentielles Triagetool: Eine prospektive Observationsstudie

\section{S. Schnaubelt, J. Oppenauer, F. Eibensteiner}

Universitätsklinik für Notfallmedizin, Medizinische Universität Wien, Wien/A;

Hintergrund: Die zeitgerechte Versorgung des akuten Koronarsyndroms (ACS) stellt einen Eckpfeiler der Notfallmedizin dar. Optimierte Abläufe bieten auch in der Akutsituation bei schneller Herzkatheteruntersuchung und koronarer Intervention die Möglichkeit eines guten Outcomes. Jedoch bleiben Wartesituationen und Reihenfolgenbildung bei einem gleichzeitigen Mehrauftreten von ACS in einem zuständigen Interventionszentrum, sowie die korrekte Einschätzung von ambulanten PatientInnen mit Thoraxschmerz hinsichtlich ihres Risikos für ein ACS weiterhin herausfordernd.

Methode: An der Univ.Klinik für Notfallmedizin, Medizinische Universität Wien, wurden 2020-2021 58 PatientInnen (65 (61-69) Jahre; 60 \% männlich) welche sich wegen akutem Thoraxschmerz in Behandlung befanden und infolgedessen eine Herzkatheteruntersuchung erhielten, in die Studie eingeschlossen. Es wurden der Ankle-Brachial-Index (ABI) und die Pulswellengeschwindigkeit (PWV) als Marker der Gefäßsteifigkeit und des allgemeinen kardiovaskulären Risikos gemessen.

Ergebnisse: Preliminary Results: PatientInnen mit pathologischen PWV Werten wiesen signifikant höhere Troponin-, CK- und proBNP Werte auf. PatientInnen mit einem interventionsbedürftigen ACS zeigten signifikant höhere PWV Werte in Bezug auf die Untersuchungs- und Interventionsdetails (längere Untersuchungs- und Strahlendauer, höhere Kontrastmittelmenge, höhere Strahlungsdosis); diese Assoziationen fanden sich auch in den entsprechenden Korrelationen wieder. Ein eigens berechneter Untersuchungs- und Interventions-Komplexitäts-Score korrelierte ebenfalls direkt mit der PWV.

Zusammenfassung: Die nicht-invasive und schnelle Messung von PWV bei Thoraxschmerz-PatientInnen, welche sich entweder auf einer Warteposition für eine Herzkatheteruntersuchung oder in Abklärung bzgl. ACS befinden, kann wertvolle Informationen über den allgemeinen Gefäßzustand und das kardiovaskuläre Risiko bieten. PWV Werte zeigen auch eine Assoziation mit der Dauer und Komplexität der Herzkatheteruntersuchung und -intervention. Somit könnte die PWV in Zukunft nach entsprechender Validierung ein zusätzliches potentielles Triagetool in ACS Algorithmen darstellen.

\section{P28}

Non-invasive oscillometry-based estimation of cardiac output can we use it in the clinical practice?

\section{A. Reshetnik, J. Gjolli, M. van der Giet, F. Compton}

Medizinische Klinik IV, Klinik für Nephrologie, Campus Benjamin Franklin, Charité - Universitätsmedizin Berlin, Berlin; 


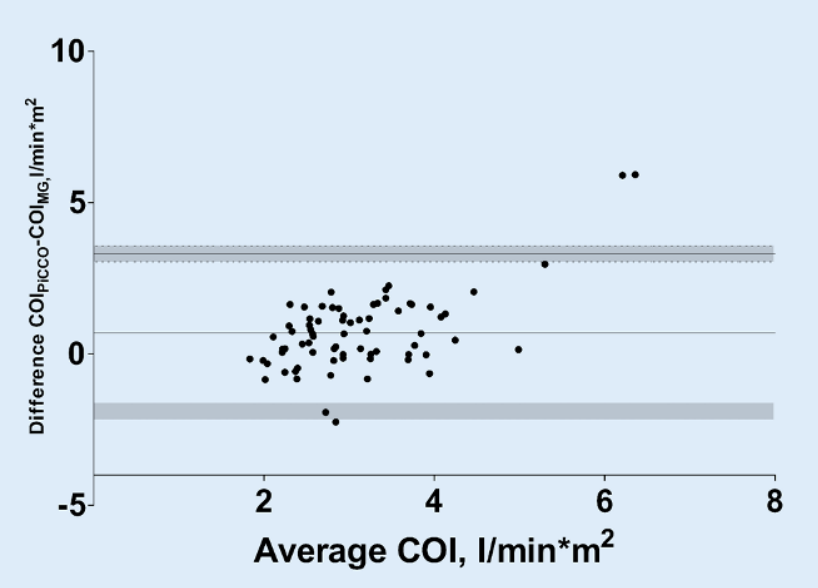

Fig. 1 | P28 $\Delta$

Objective: Cardiac output (CO) monitoring is recommended for perioperative goal-directed hemodynamic therapy, hemodynamic assessment of critically ill patients, and to monitor response to therapeutic interventions. While invasive indicator dilution techniques remain the reference methods for CO measurement, there is a currently unmet need for non-invasive methods in order to simplify CO measurement, reduce complications related to invasive procedures such as pulmonary artery catheter placement, and expand the application field towards emergency room, non-intensive care or outpatient settings.

Method: We evaluated the performance of a non-invasive oscillometry-based CO estimation method (Mobil-O-Graph (MG; I.E. M., Stolberg, Germany)) compared to transpulmonary thermodilution with the $\mathrm{PiCCO}^{\circ}$ system (Pulsion Medical Systems, Feldkirchen, Germany) as a reference method. To assess the agreement between devices, we used BlandAltman analysis. 4-quadrant plot was used to visualize the ability of MG to track CO changes after a fluid challenge. Trending analysis of CO trajectories was used to compare $\mathrm{MG}$ and $\mathrm{PiCCO}^{\circ}$ calibrated pulse wave analysis over time (six hours).

Results: We included 40 patients from the medical intensive care unit at the Charité Universitaetsmedizin Berlin, Campus Benjamin Franklin between November 2019 and June 2020. Median age was 73 years. $40 \%$ of the study population was male; $98 \%$ was ventilator-dependent and $75 \%$ vasopressor-dependent at study entry. The mean of the observed differences for the cardiac output index (COI) was $0.71^{*} \mathrm{~min}^{-1 *} \mathrm{~m}^{-2}$ and the lower and upper $95 \%$ limit of agreement were -1.9 and $3.31^{*} \mathrm{~min}^{-1 *} \mathrm{~m}^{-2}$, respectively (• Fig. 1).

The $95 \%$ confidence interval for the limits of agreement was $\pm 0.261^{\star} \mathrm{min}^{-1 *} \mathrm{~m}^{-2}$, the percentage error $83.6 \%$. We observed concordant changes in $\mathrm{CO}$ with $\mathrm{MG}$ and $\mathrm{PiCCO}^{\circ}$ in $50 \%$ of the measurements after a fluid challenge and over the course of six hours.

Conclusion: $\mathrm{CO}$ calculation with a novel oscillometry-based pulse wave analysis method is feasible and replicable in critically ill patients. However, we did not observe clinically applicable agreement between MG and thermodilution assessed with established evaluation routine using BlandAltman approach and with trending analysis methods. In summary, we do not recommend to use this method in critically ill patients currently. As the basic approach is promising and the performance of $\mathrm{CO}$ calculation with MG very simple, further studies should be performed in hemodynamic stable patients and in critical care setting in order to perform further adjustments to the algorithm.

\section{Respiratorisches Versagen}

\section{P29}

Perkutane Dilatationstracheotomie - Einwegbronchoskop vs Mehrwegbronchoskop - eine prospektiv randomisierte Studie (TraSUB)

\section{P. A. Tariparast, J. Grensemann, A. Brockmann, S. Kluge}

Klinik für Intensivmedizin, Zentrum für Anästhesiologie und Intensivmedizin, Universitätsklinikum Hamburg-Eppendorf, Hamburg;

Hintergrund: Während einer perkutanen dilatativen Tracheotomie erfolgt oft eine Kontrolle mittels Bronchoskopie. Hierfür stehen neben wiederverwendbaren Bronchoskopen (CONV) auch Einwegbronchoskope (SUB) zur Verfügung. Die Verwendung von SUB könnte das Risiko einer KreuzKontamination (z. B. durch multiresistente Erreger, SARS-CoV-2) reduzieren, jedoch wurde die Verwendung für die PDT bisher nicht evaluiert. Daher wurden in dieser Studie SUB im Vergleich zu CONV für die Verwendung während einer PDT untersucht.

Methode: In dieser prospektiven Nichtunterlegen-heitsstudie wurden 46 Patienten zur PDT 1:1 auf SUB (Ambu aScope 4 Broncho Regular, Außendurchmesser (OD): $5 \mathrm{~mm}$, Ambu A/S, Ballerup Dänemark) oder CONV (Olympus BF-P60, OD: 4,9 mm, Olympus Medical Systems Corp., Tokyo, Japan) randomisiert. Die Visualisierung von Trachealstrukturen, bewertet auf 4-Punkt-LikertSkalen, war der primäre Endpunkt. Darüber hinaus wurden die Qualität der Beatmung, die Handhabung der Geräte sowie Veränderungen im Gasaustausch der Patienten sowie die Kosten verglichen.

Ergebnisse: Es bestand bei mittleren Visualisierungs-werten (niedrigere Werte besser) von 4,1 (95\% Konfidenzintervalle: 3.9;4.3) für SUB vs.4.1 (4.0;4.2) für CONV und für die Beatmung mit 2,6 (2,0;3,1) für SUB vs.2,4 $(2,1 ; 2,7)$ für CONV jeweils eine Nicht-Unterlegenheit. Es konnten keine signifikanten Unterschiede hinsichtlich Handhabung (SUB: 1.2 (1.0;1.4), CONV: $1.3(1.1 ; 1.6))$, Blutgasanalysen und respiratorischen Variablen gefunden wurden. Die Kostenanalyse in unserer Institution ergab $93 €$ pro konventionelle Bronchoskopie versus 232,50 € mit SUB, dabei erfolgte keine Berücksichtigung einer Infektion durch eine Kreuzkontamination mit dem wieder-verwendbaren Gerät.

Zusammenfassung: Visualisierung, Beatmung und Handling der SUB sind analog zu Mehrwegbronchoskopen zu bewerten, womit ein Einsatz während einer PDT erfolgen kann. In unserem Zentrum war der Einsatz der SUB teurer, jedoch hängen die Kosten von vielen Faktoren ab, so dass jedes Zentrum diese individuell erheben sollte.

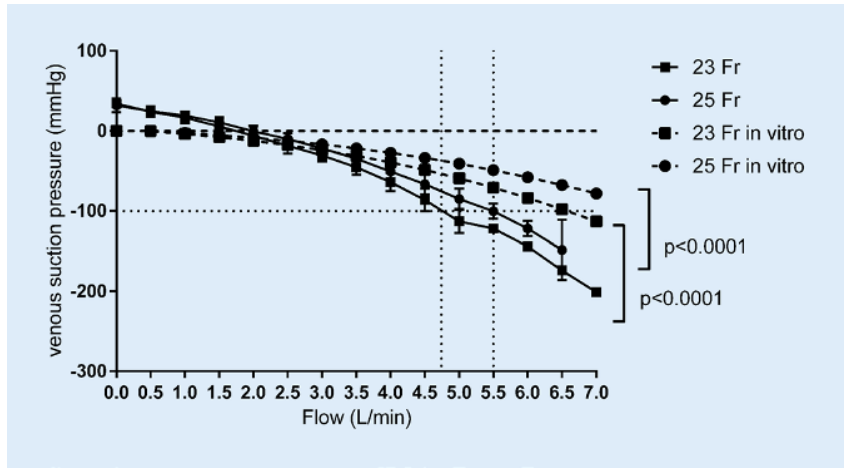

Fig. 1 | P30 4 
P30

\section{In vivo pressure-drop of venous cannulas during veno-venous extracorporeal membrane oxygenation}

O. Robak, T. Grafeneder-Weissteiner, P. Schellongowski, A. Bojic, C. Paschen", A. Hermann, T. Staudinger

Intensivstation 13i2, Universitätsklinik für Innere Medizin I, Medizinische Universität Wien, Wien/A; 'Universitätsklinik für Innere Medizin III, Medizinische Universität Wien, Wien/A

Objective: Extracorporeal lung support includes the risk of hemolysis caused by negative suction pressures in the case of high blood flow rates. Previous studies have paid little attention to the relationship between blood flows and pressure-drops. Manufacturers of extracorporeal gas exchange devices measure the pressure-drop across drainage cannulas for their products in vitro, using water instead of blood. Clinical experience suggests that hemolysis occurs in vivo already at a much lower flow rates The aim of this clinical study was therefore to systematically collect and analyze the in vivo pressure-drop relationship for VV-ECMO cannulas observed in the clinical routine.

Method: Prospective, observational study at a medical intensive care unit at a tertiary care, university-level hospital. 14 patients that had to be put on veno-venous extracorporeal membrane oxygenation (VV-ECMO) for severe ARDS were prospectively included.

Results: In vitro, the 25 Fr drainage cannula pressure drops below a critical level of around $-100 \mathrm{mmHg}$ at a flow rate of $7.9 \mathrm{~L} / \mathrm{min}$, the $23 \mathrm{Fr}$ drainage cannula at $6.6 \mathrm{~L} / \mathrm{min}$. In the clinical setting, critical suction pressures were reached at much lower flow rates $(5.5$ and $4.7 \mathrm{~L} / \mathrm{min} ; p<0.0001$, non-linear regression). The in vitro data largely overestimate the safely achievable flow rates in daily clinical practice by $2.4 \mathrm{~L} / \mathrm{min}$ (or $44 \%, 25 \mathrm{Fr}$ ) and $1.9 \mathrm{~L} / \mathrm{min}$ (or $41 \%, 23 \mathrm{Fr}$; $\bullet$ Fig. 1).

Conclusion: In vivo measurement of pressure-drop curves of venous drainage cannulas differed significantly from in vitro derived measurements during veno-venous membrane oxygenation as the latter largely underestimate the resulting pressure-drop.

\section{P31}

\section{Ischämische Komplikationen bei extrakoporaler Membranoxygenierung}

\section{Gärtner, J. Fichte', H.-B. Hopf}

Zentrale Abteilung für Anästhesie und perioperative Medizin, Asklepios Kliniken Langen-Seligenstadt GmbH, Langen; 'Klinik für Anästhesiologie, Intensivmedizin und Schmerztherapie, Universitätsklinikum Frankfurt, Frankfurt;

Hintergrund: Ischämische Komplikationen der Extremitäten und inneren Organe sind gefürchtete und potentiell tödliche Komplikationen wäh-

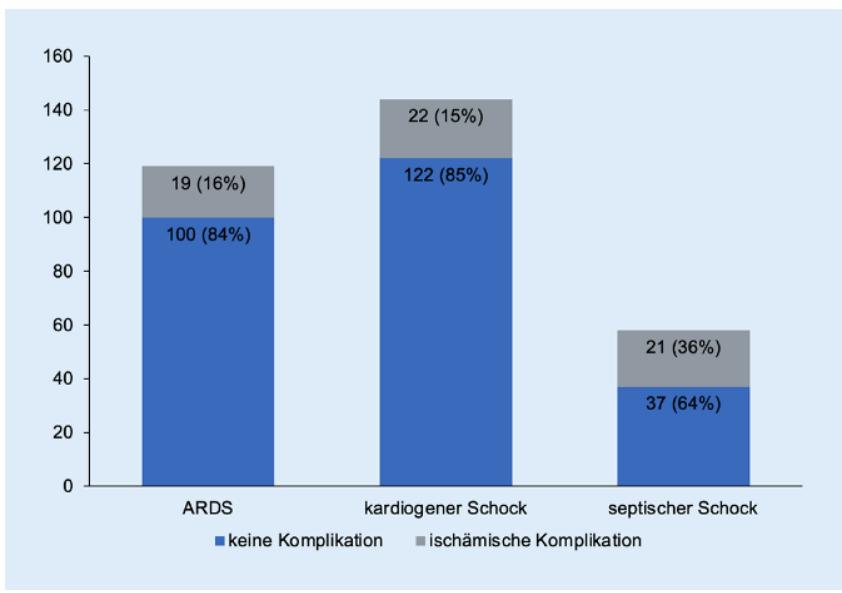

Abb. 1 |P31 4 rend einer Therapie mit extrakorporaler Membranoxygenierung (ECMO). Wir analysierten die Art ischämischer Komplikationen während ECMOTherapie, unterteilt in Extremitäten, mesenteriale, kardiale und neurologische Ischämien, mit der Frage der Inzidenz und Identifizierung von Risikofaktoren.

Methode: Wir untersuchten retrospektiv die Daten von 348 Patienten, die von 01.04.2011 bis 31.03.2020 auf unserer Intensivstation mit V-V, V-A oder V-V-A ECMO behandelt wurden. 321 Patienten mit Diagnose ARDS, kardiogener oder septischer Schock wurden eingeschlossen.

Primäre Zielvariable war die Art der ischämischen Komplikation. Weitere Variablen: Alter, Geschlecht, Diagnose, Serumlaktat $24 \mathrm{~h}$ vor und direkt nach Auftreten der Ischämie, Dauer Krankenhaus- und Intensivstationsaufenthaltes, Dauer ECMO-Therapie und Beatmung, Modus, SAPS II, SOFA Score, Mortalität, Einsatz von Hämodialyse und Tracheotomie, Infektionen während des Intensivaufenthalts und CPR vor ECMO-Therapie. Statistik: Chi-Quadrat-Test, Mann-Whitney U Test, Kruskal-Wallis Test; multivariate logistische Regression zur primären Zielvariable mit Angabe von Odds Ratio und Konfidenzintervallen. Signifikanzlevel: $p<0.05$. Ergebnisse: Bei 62/321 Patienten (19,3\%) wurde eine ischämische Komplikation diagnostiziert. Die häufigsten Lokalisationen waren Extremitäten $(n=32)$ und mesenterial $(n=21)$. Patienten, mit Diagnose eines septischen Schocks hatten die höchste Rate an Ischämien. Es wurde kein Unterschied im Überleben zwischen Patienten mit und ohne Komplikation gefunden $(p=1,0)$. Die Wahl des ECMO-Modus hatte keinen Einfluss auf die Inzidenz der Ischämien $(p=0,063)$. Es konnten ein Alter $\geq 50$ Jahren $(p=0,029 ; \mathrm{OR}=2,793 ; \mathrm{KI}=1,109-7,033)$, der Einsatz einer Hämodialyse $(p=0,003 ; \mathrm{OR}=3,283 ; \mathrm{KI}=1,513-7,124)$ und die Diagnose ,septischer Schock" $(p=0,049 ; \mathrm{OR}=2,144 ; \mathrm{KI}=1,003-4,583)$ als Prädiktoren für ischämische Komplikationen identifiziert werden (• Abb. 1).

Zusammenfassung: Ischämische Komplikationen sind während einer ECMO-Therapie häufig. Ein Alter $\geq 50$ Jahren, der Einsatz von Hämodialyse und Diagnose eines septischen Schocks waren Prädiktoren für ischämische Komplikationen. Es wurde keine Korrelation zwischen ECMOModus und ischämischen Komplikationen gefunden. Es gab keinen Unterschied im Überleben zwischen Patienten mit und ohne Ischämie.

\section{P32}

\section{Zerebrovaskuläre Autoregulation und neurokognitives Outcome beim akuten Lungenversagen}

\section{Fischer, U. Kahl, Y. Yu, A. Nierhaus, D. Frings, B. Sensen, A. Daubmann',} S. Kluge

Klinik für Intensivmedizin, Zentrum für Anästhesiologie und Intensivmedizin, Universitätsklinikum Hamburg-Eppendorf, Hamburg; 'Institut für Medizinische Biometrie und Epidemiologie (IMBE), Universitätsklinikum Hamburg-Eppendorf, Hamburg;

Hintergrund: Die vorgestellten Ergebnisse sind Teil einer prospektiven Beobachtungsstudie, in der untersucht wurde, 1) ob es bei Patienten mit ARDS (acute respiratory distress syndrome) und primärer Hyperkapnie $\left(\mathrm{PaCO}_{2}>50 \mathrm{mmHg}, \mathrm{pH}<7.35\right)$ im Vergleich zu Patienten mit rein hypoxämischem ARDS zu einer stärkeren Beeinträchtigung der zerebrovaskulären Autoregulation (CVA) kommt (veröffentlicht; PMID 33725209) und 2) ob eine Beeinträchtigung der CVA während der Akutphase des ARDS mit ungünstigem funktionellen Outcome, verminderter gesundheitsbezogener Lebensqualität (HRQoL) und selbst-berichteter kognitiver Funktionseinschränkung einhergeht.

Methode: Patienten mit ARDS ohne Hinweis für eine chronische Hyperkapnie oder zerebrovaskuläre Vorerkrankungen wurden eingeschlossen. Basierend auf zerebraler Oxygenierung, gemessen mit Nahinfrarotspektroskopie, sowie invasivem Blutdruck wurde ein Autoregulationsindex berechnet. Die CVA wurde über einen Zeitraum von 60 min innerhalb von 6 Tagen nach Diagnose des ARDS aufgezeichnet. Selbst-berichtete kognitive Fähigkeiten und die HRQoL wurden anhand des Cognitive Failures Questionnaire sowie des Short-Form 36 Fragebogens erhoben.

Ergebnisse: Zwischen Dezember 2018 und Dezember 2019 wurden $66 \mathrm{~Pa}$ tienten mit mildem $(n=10)$, moderatem $(n=28)$ und schwerem $(n=28)$ 
ARDS eingeschlossen. Der mittlere Autoregulationsindex befand sich während $25 \% \pm 19$ des Aufzeichnungszeitraums im pathologischen Bereich. Die Letalität nach 30 Tagen betrug 40,9\% $(n=27)$; nach 90 Tagen waren $50,0 \%(n=33)$ aller Studienteilnehmer verstorben. Selbst-berichtete kognitive Fehler wurden mit 30 Punkten angegeben; die körperliche HRQoL betrug im Mittel 33,2\% $\pm 12,1$; die psychische HRQoL lag bei $45,7 \% \pm 11$. Patienten mit eingeschränkter CAR wiesen eine signifikant niedrigere Überlebenswahrscheinlichkeit nach 30 ( $p=0,034$; LogrankTest) sowie nach 90 Tagen $(p=0,025)$ auf als Patienten mit intakter CAR. Zusammenfassung: Diese Ergebnisse weisen darauf hin, dass Patienten mit beeinträchtigter CAR während der Akutphase des ARDS eine geringere Überlebenswahrscheinlichkeit nach 30 sowie nach 90 Tagen aufweisen im Vergleich zu Patienten mit intakter CAR. Eine mögliche Assoziation zwischen CAR und Outcome nach ARDS und potentielle, sich daraus ableitende Interventionsmöglichkeiten sollten in größeren Studien mit konfirmatorischem Ansatz bestätigt werden

\section{P33}

Nasotracheale versus orotracheale Intubation bei kritisch kranken Patienten - eine retrospektive Auswertung (NaTra-R)

\section{S. Gilmour, P. A. Tariparast, A. Brockmann, S. Kluge, J. Grensemann}

Klinik für Intensivmedizin, Zentrum für Anästhesiologie und

Intensivmedizin, Universitätsklinikum Hamburg-Eppendorf, Hamburg;

Hintergrund: Kritisch kranke Patienten werden zur maschinellen Beatmung orotracheal (OTI) oder nasotracheal intubiert (NTI). Wir untersuchten den Einfluss einer NTI versus OTI in Bezug auf Spontanatmung, analgosedierender Medikation und Sedierungsgrad. Die NTI wurde bis vor circa 25 Jahren häufig verwendet und dann fast ausschließlich von der OTI ersetzt.

Methode: In dieser retrospektiven Studie wurden Patienten unserer Intensivstationen (140 Betten) von 2018-2020, die für einen Zeitraum von mindestens 7 Tagen invasiv beatmet wurden, anhand des Atemwegszugangs der OTI- oder der NTI-Gruppe zugeordnet. Aus der elektronischen Datenbank wurden u. a. Beatmungsform, Dosis der Analgosedierung (Sufentanil, Propofol, Midazolam) und Richmond-Agitation-Sedation-Scale (RASS) an Tag 1, 5, und 10 nach Intubation extrahiert und mittels gemischter linearer Modelle analysiert.

Ergebnisse: Es wurden 79 Patienten mit NTI und 455 mit OTI ausgewertet, die sich in Bezug auf Krankheitsschwere (SAPS-DRG NTI: $41 \pm 10$ vs. OTI: $44 \pm 13$, n.s.) und demographische Parameter nicht unterschieden. Die NTI Gruppe atmete an Tag 1 bis Tag 10 jeweils häufiger spontan (Mittelwert, $95 \%$ Konfidenzintervalle): an Tag 1 atmeten $28 \%(21 ; 24)$ der NTI Gruppe spontan und $8 \%(6 ; 11)$ der OTI Gruppe, $p<0,001$, an Tag 5 waren es $42 \%(36 ; 49)$ versus $27 \%(24 ; 30), p<0,001$ und an Tag $1048 \%$ $(40 ; 56)$ versus $37 \%(24 ; 41), p<0,05$. Patienten der NTI Gruppe waren weniger stark sediert: RASS Tag $1:-2,8(-3,1 ;-2,5)$ versus $-3,2(-3,4 ;-3,1)$, Tag 5: $-2,0(-2,3 ;-1,7)$ versus $-2,6(-2,7 ;-2,5)$, Tag $10:-1,5(-1,9 ;-1,1)$ versus $-2,0(-2,2 ;-1,9)$, jeweils $p<0,01$ und erhielten weniger Sufentanil: an Tag 1 waren es $709 \mu \mathrm{g} / \mathrm{d}$ in der NTI Gruppe $(514 ; 904)$ versus $1135 \mu \mathrm{g} / \mathrm{d}$ in der OTI Gruppe $(1054 ; 1217), p<0,001$, an Tag $5624(428 ; 819)$ versus 885 (803;966), $p<0,05$ und an Tag 10413 (195;631) versus 705 (614;795), $p<0,05$. Ein Unterschied für Propofol und Midazolam bestand nicht.

Zusammenfassung: Patienten der NTI-Gruppe erhielten im Vergleich zu Patienten der OTI-Gruppe weniger Sufentanil, wurden seltener kontrolliert beatmet und waren laut der RASS weniger stark sediert. Patienten, bei denen eine NTI möglich ist, könnten von der häufigeren unterstützten Beatmung profitieren. Ob die NTI einen aktuellen positiven Effekt auf das Outcome hat, sollte in weiteren prospektiven Studien erneut untersucht werden.

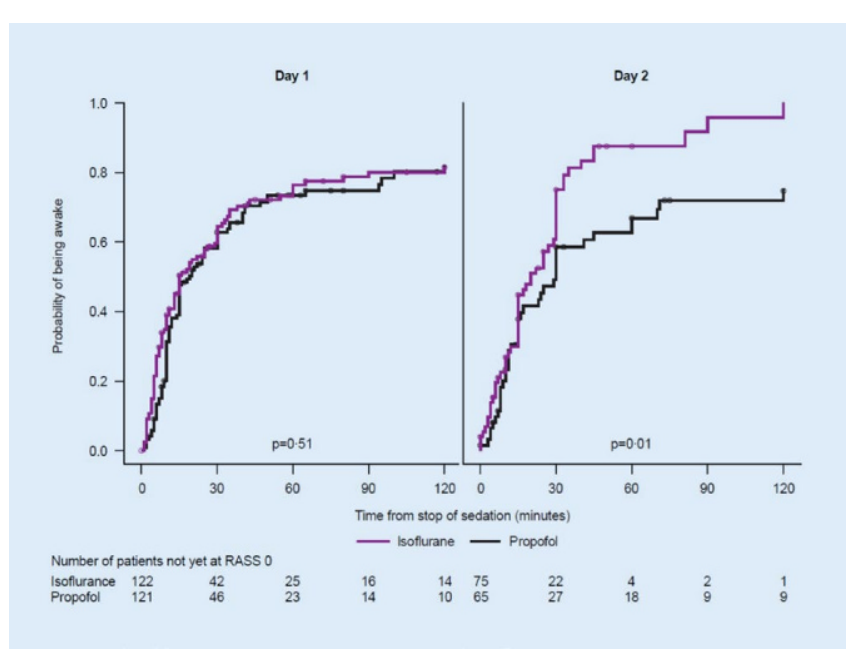

Fig. 1 | P34 A

\section{P34}

First results from SEDACONDA: A phase-III multicenter randomized controlled trial evaluating the efficacy and safety of isoflurane via AnaConDa for sedation in invasively ventilated patients

A. Meiser, T. Volk, U. Günther ${ }^{1}$, R. Knafeli ${ }^{2}$, M. Bellgardt ${ }^{3}$, P. Sackey ${ }^{4}$, Sedaconda study group ${ }^{5}$

Klinik für Anästhesiologie, Intensivmedizin und Schmerztherapie, Universitätsklinik Homburg, Universität des Saarlandes, Homburg/ Saar; ${ }^{1}$ Klinik für Anästhesiologie, Intensivmedizin, Notfallmedizin und Schmerztherapie, Klinikum Oldenburg, Carl von Ossietzky Universität, Oldenburg; ${ }^{2} \mathrm{MICU}$, Klinicni oddelek za interno intenzivno medicine, University Medical Center, Ljubljana/SLO; ${ }^{3}$ Klinik für Anaesthesiologie und Intensivmedizin, St. Josef-Hospital, Ruhr-Universität, Bochum; ${ }^{4}$ Department of Physiology and Pharmacology, Karolinska Institutet, Stockholm $/ S_{;}{ }^{5}$ T. Becher, B. Bein, P. Biever, R. Bogdanski, H. Bracht, M. Drüner, A. Faltlhauser, A. Geise, P. Gude, Hamsen, P. Kellner, A. Kermad, K. Kogelmann, R. Kram, A. Markota, W. Meermeier, M. Podbregar, K. Schwarzkopf, J. Soukup,

S.C. Thal, H. Vogelsang, J. Wallenborn, C. Waydhas

Objective: The aim of this phase III randomized controlled registration trial was to evaluate efficacy and safety of sedation with isoflurane administered via the anesthetic conserving device.

Method: After ethical approval (Ärztekammer des Saarlandes, 11/17), trial registration (Eudra CT\#:2016-004551-67), and written informed consent, 21 German and 3 Slovenian study sites included 300 patients, randomized to either isoflurane (Sedaconda ${ }^{\circ}$, Sedana Medical, Danderyd, Sweden) or propofol for up to $48 \pm 6$ hours or until extubation. Richmond Agitation and Sedation Scale (RASS) Scores were recorded two-hourly or before dose changes. Sedation efficacy was assessed as the proportion of time within RASS- 1 to RASS - 4 (primary endpoint) with a pre-defined noninferiority margin of $-15 \%$ relative to the mean for propofol. Secondary efficacy endpoints included opioid dosing, wake-up and extubation times. Safety endpoints included adverse events, lab values, vital signs, ventilator, ICU and delirium free days, and proportion of time with spontaneous breathing.

Results: 301 critically ill patients were enrolled (isoflurane vs propofol, mean \pm SD: age $66 \pm 12$ vs $64 \pm 13$ years, SAPS-II: $42 \pm 17$ vs $44 \pm 19$, emergencies $65 \%$ vs $65 \%$ ). The percentage of time within the target RASS range was similar for both groups (mean $\langle\mathrm{CI}\rangle: 91 \%\langle 87-95\rangle$ vs $91 \%\langle 87-95\rangle$; - Fig. 1).

Wake-up times were shorter and more predictable after isoflurane with significance on day 2 (median [IQR]: 20 [10-30] vs 30 [11-120], $p=0.01$ ). Extubation times were also shorter though not significantly (30 [10-136] vs 40 [18-125] $\mathrm{min}, p=0.21)$. Opioid requirements were lower $(0.23<0.12-$ 
$0.33\rangle$ vs $0.32\langle 0.21-0.42\rangle \mathrm{Mo}-\mathrm{Eq} / \mathrm{kg} / \mathrm{h}, p=0.004)$ and spontaneous breathing was more frequent on day 1 ( $50 \%$ vs $37 \%, p=0.013$ ). Safety endpoints did not show any major differences of note.

Conclusion: SEDACONDA is efficacious as a primary sole sedative, in the same efficacy range as propofol. Opioid requirements are lower and spontaneous breathing more common during isoflurane than during propofol sedation. The times to wake-up and extubation are short and predictable. No new safety concerns arose for isoflurane given in subanesthetic doses for sedation in the ICU.

\section{Akut- und Notfallmedizin}

\section{P35}

\section{Early identification of patients with chest pain at very low risk of acute myocardial infarction using clinical information and ECG only}

K. Tscherny, C. Kienbacher, V. Fuhrmann, W. Schreiber, H. Herkner, D. Roth Universitätsklinik für Notfallmedizin, Medizinische Universität Wien, Wien/A;

Objective: A considerable proportion of patients with angina-like symptoms in an emergency department have very low pre-test probability for acute myocardial infarction (AMI). Numerous algorithms exist for the exclusion of AMI, usually including laboratory tests. We aimed to investigate whether patients with very low risk can safely be identified by ECG and clinical information without biomarker testing, contributing to saving time and costs.

Method: Prospective diagnostic test accuracy study. We included all consecutive patients presenting with angina at the department of emergency medicine of a tertiary care hospital during a one-year period. Using clinical information without biomarker testing and ECG, the "Mini-GRACE score", based on the well-established GRACE-score without using laboratory parameters was calculated. In a cohort design we compared the index test Mini-GRACE to AMI as reference standard in the final diagnosis using standard measures of diagnostic test accuracy.

Results: We included 2,755 patients ( $44 \%$ female, age $44 \pm 17$ years). Acute myocardial infarction was diagnosed in $103(4 \%)$ patients, among those $44 \%$ with STEMI. Overall 2,562 patients $(93 \%)$ had a negative 'MiniGRACE', four ( $0.2 \%)$ of these patients had myocardial infarction, and this results in a sensitivity of $96.1 \%$ (95\% CI 90.4-98.9\%), specificity $96.5 \%$ (95.7-97.1\%), positive predictive value 51.3\% (46.3-56.3\%) and negative predictive value $99.8 \%$ (99.6-99.9\%). Model performance according to C statistic (0.90) and Brier score (0.0045) was excellent. In rule-out patients 30 -day mortality was $0.3 \%$, and one-year mortality was $0.8 \%$.

Conclusion: Patients with very low risk of AMI can be identified with high certainty using clinical information without biomarker testing and ECG. Cardiac biomarkers might be avoided in such cases, potentially leading to a significant cost reduction.

\section{P36}

\section{Can 90 seconds of teaching improve the quality of basic life support and AED-use in the general population}

F. Alemdar, M. Brand, H. de Bakker, H.-J. Trappe

Medizinische Klinik II - Kardiologie und Angiologie, Marien Hospital Herne, Ruhr-Universität Bochum, Herne;

Objective: The rate and quality of basic life support (BLS) performed in the general population is-despite national programmes and activities of institutions-insufficient. The aim of this study was to asses, if 90 seconds of live teaching is enough to show a significant improvement of the quality of BLS and increase of correct AED-use.

Method: On different public places (swimming pools, schools, cafes) we set up a scenario with a training manikin (AmbuMan Airway Instrument) dressed in normal clothes and placed an AED-Trainer (Physiocon-

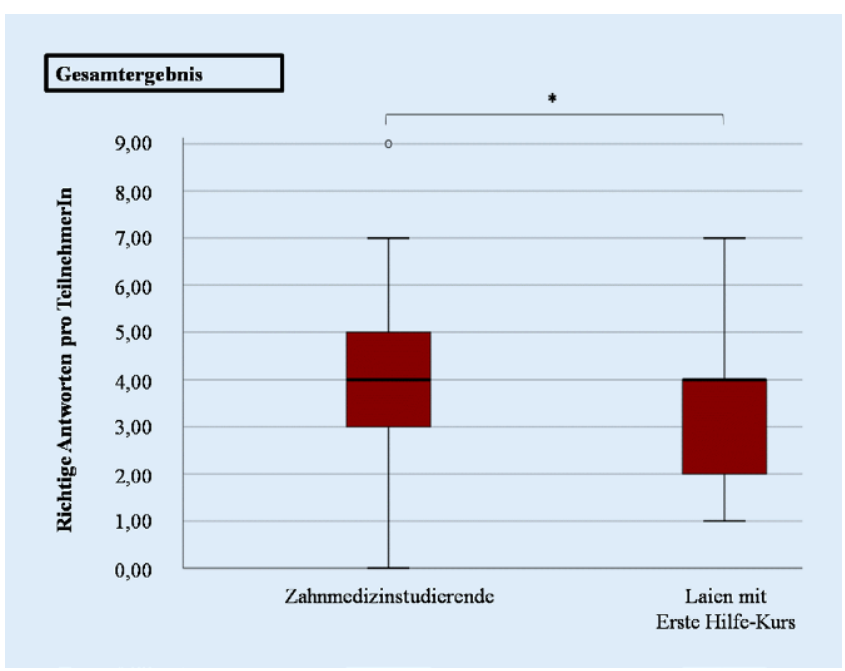

Abb. 1 | P37

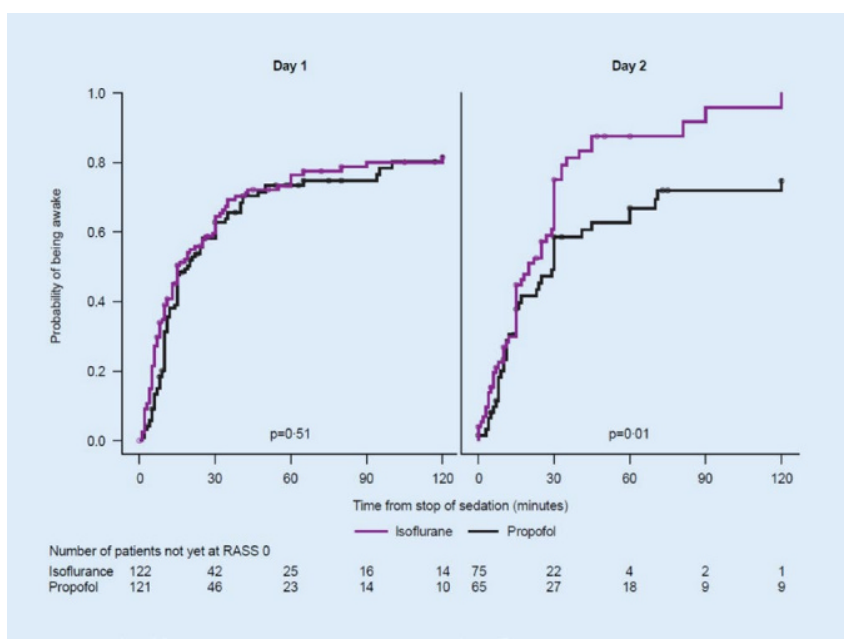

Abb. 2 |P37 4

trol Lifepak CR-T) close to the manikin. We asked passersby: Imagine a 50 year old man collapsed in front of you and lies here. What would you do? Using a predefined protocol we analysed the order, point of time and quality of measures of the first responders. After the first session, we defined a control and intervention group to rule out bias by anxiety. The allocation to the group was defined by lot. For the intervention group we did a standardised teaching for 90 seconds using a poster and short demonstration. The control group had 90 seconds to reflect their action.

We started a second session for both groups with the same setting and evaluation protocol. The results of both sessions and groups were compared and analysed.

Results: Of 189 participants (age: $18-80 y$ ) only $22 \%$ did a correct assessment of the cardiac arrest in time. $84 \%$ called the correct emergency number. Only $17 \%$ did effective chest compressions. $28 \%$ brought the patient in a stable lateral position without compressions. The AED was used by only $10 \%$ of all first responders.

After the short teaching we saw a significant $(p<0,01)$ improvement of correct assessment of the cardiac arrest in time $(81 \%)$ and effective chest compressions ( $80 \%)$. Only $1,2 \%$ brought the patient in a stable lateral position without compressions. The AED was used by $68 \%$ of all responders with a correct use of $92 \%$.

No significant change was seen in the control group 
Conclusion: In your study we could show that 90 seconds of focused live teaching can dramatically improve the quality of basic life support and rate of AED-use. All first-responders that used the AED without prior information were able to deliver the shock correct and in time. Although the study is ongoing to complete at least 500 probands we have strong evidence that a mandatory regular BLS-training/knowledge-refreshing could have a strong impact on quality of first aid and outcome of patients with outof-hospital cardiac arrest

\section{P37}

\section{Reanimationskenntnisse von Zahnmedizinstudierenden im Vergleich zu medizinischen Laien mit Erste-Hilfe-Kurs}

\section{G. Schiehser, M. Felfernig, H. Fischer}

Zentrum für Akut-, Notfall- und Intensivmedizin, Sigmund Freud Privat Universität, Wien/A;

Hintergrund: Der plötzliche Herzstillstand ist mit einer Inzidenz von 350.000-700.000 Personen/Jahr eine der führenden Todesursachen in Europa. Frühzeitige Reanimationsmaßnahmen (CPR) und Defibrillation erhöhen die Wahrscheinlichkeit einen Herzstillstand bis zur Krankenhausentlassung zu überleben auf bis zu 50-70\%.

$57 \%$ der Zahnmediziner berichten von bis zu 3 Notfällen pro Jahr in ihrer Ordination. Laut Literatur können in ihrer Berufslaufbahn darunter 5-7 lebensbedrohliche Notfälle sein. Daher sollten auch Zahnmediziner mit der Erstversorgung eines Herzstillstands vertraut sein.

Methode: Diese Studie erfolgte im Rahmen einer Masterthesis. 50 Zahnmedizin- und 50 Psychologiestudierende als medizinische Laien wurden in die Studie eingeschlossen. Alle Teilnehmer absolvierten in den vergangenen 5 Jahren einen Erste-Hilfe(EH)-Kurs. Die Reanimationskenntnisse wurden anhand eines Fragebogens erhoben.

Ziel der Studie war herauszufinden, ob ein Unterschied hinsichtlich der Reanimationskenntnisse zwischen Zahnmedizinstudierenden und medizinischen Laien besteht.

Ergebnisse: Zahnmedizinstudierende beantworteten mit durchschnittlich 4,2 von 9 Fragen $(46,6 \%)$ signifikant mehr Fragen richtig als medizinische Laien ( 3,5 von 9 Fragen, $39,3 \%)(p=0,035$; $\bullet$ Abb. 1$)$.

$34 \%$ der Zahnmediziner und $24 \%$ der Laien wussten wie man einen Herzstillstand erkennt $(p=0,271) .22 \%$ der Zahnmediziner im Vergleich zu $30 \%$ der Laien kannten die richtige Frequenz der Thoraxkompressionen $(p=0,362) .86 \%$ respektive $58 \%$ kannten das richtige Verhältnis von Herzdruckmassagen zu Beatmungen bei Erwachsenen $(p=0,002)$.

Die Frage nach ihrer Bereitschaft bei einer fremden Person eine CPR durchzuführen, beantworteten $74 \%$ der Zahnmedizinstudierenden mit "Ja“ und $22 \%$ mit „Vielleicht“. Im Vergleich dazu antworteten $46 \%$ der Laien mit „Ja“ und $36 \%$ mit „Vielleicht“ $(p=0,002)$.

Es zeigte sich ein Hinweis darauf, dass Teilnehmer, deren letzter EH-Kurs maximal 1 Jahr zurücklag, mit 55,5\% mehr Fragen richtig beantworten konnten als andere Teilnehmer ( $p=0,038$; $\bullet$ Abb. 2).

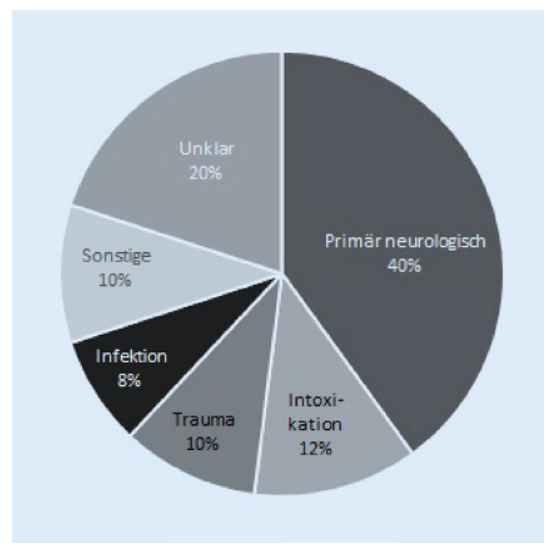

Abb. $1 \mid$ P38 4

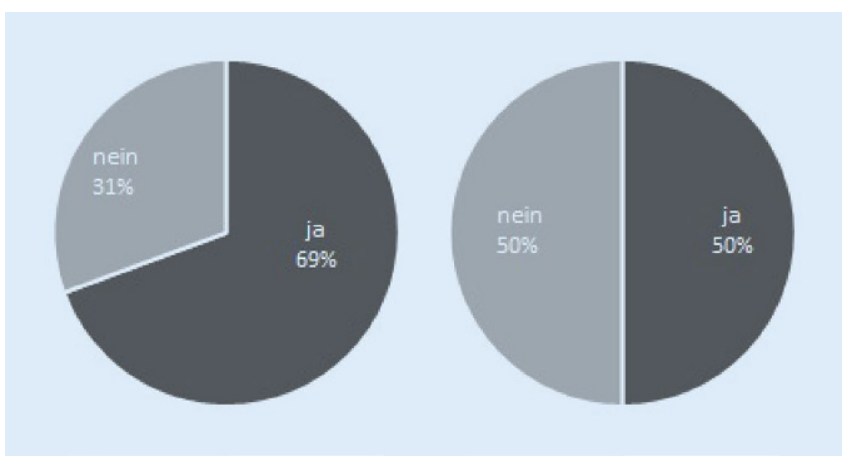

Abb. $2 \mid$ P38 4

Zusammenfassung: Zahnmedizinstudierende wiesen bessere CPR-Kenntnisse als Laien auf. Insgesamt war das CPR-Wissen in beiden Gruppen jedoch mangelhaft. Teilnehmer, die innerhalb des vorangegangenen Jahres einen EH-Kurs absolviert hatten, konnten tendenziell mehr Fragen richtig beantworten. Da CPR-Kenntnisse bereits nach wenigen Monaten abnehmen, sollte (zahn-)medizinisches Personal regelmäßig obligatorische Notfallmedizin- und Reanimationskurse absolvieren, um die Patientensicherheit zu verbessern.

\section{P38}

\section{Liegetrauma - ein unterschätztes Krankheitsbild? Ergebnisse einer Patientenkohorte aus der Notaufnahme und erste Definition}

C. Hüser, M. Hackl, V. Suárez, V. Burst, M. Bernhard ' , C. Adler ${ }^{2}$

Nephrologie, Rheumatologie, Diabetologie und Allgemeine Innere Medizin, Klinik II für Innere Medizin, Universitätsklinikum Köln, Köln; ${ }^{1}$ Zentrale Notaufnahme, Universitätsklinikum, Heinrich-Heine-Universität Düsseldorf, Düsseldorf; ${ }^{2}$ Klinik und Poliklinik für Kardiologie, Pneumologie, Angiologie und internistische Intensivmedizin, Herzzentrum, Universität zu Köln, Köln;

Hintergrund: Charakterisierung von Patienten mit Liegetrauma („LT“) und daraus resultierend Versuch einer ersten Definition des bisher nicht definierten klinischen Bildes.

Methode: Retrospektive Analyse aller Patienten, welche zwischen 07/2018 und 12/2020 mit der Diagnose „Liegetrauma“ in der Notaufnahme des Universitätsklinikums Köln vorgestellt worden sind.

Ergebnisse: Es wurden 50 Patienten (männlich: $58 \%$ ) mit einem durchschnittlichen Alter von $74 \pm 14$ Jahren (Median: 76 Jahre, Spannweite: 36103 Jahre) in die Analyse eingeschlossen. Fast alle Patienten (95\%) wurden zu Hause aufgefunden und nur ein kleiner Teil (14\%) der Patienten war $<60$ Jahre. Die erfasste Liegedauer betrug $23 \pm 26 \mathrm{~h}$ (Median 13,5 h, Spannweite: 9-96 h). Die initiale GCS lag im Mittel bei $9 \pm 5$ Punkten, $48 \%$ der Patienten waren bei Übernahme endotracheal intubiert und $20 \%$ ausgeprägt hypotherm (Temperatur $<32^{\circ} \mathrm{C}$; $\bullet$ Abb. 1).

In $40 \%$ der Fälle konnte das Indexereignis auf eine primär zerebrale Ursache zurückgeführt werden (ischämischer Schlaganfall: $20 \%$, intrakranielle Blutung: $16 \%$, Epilepsie: $4 \%$ ). Weitere häufige Ursachen des LT waren Intoxikationen (12\%) oder Trauma (10\%). Bei annähernd jedem zweiten Patienten konnte ergänzend eine Infektion (Pneumonie 36 \%, Harnwegsinfekt $12 \%$ ) dokumentiert werden und etwa jeder fünfte Patient (22\%) benötigte aufgrund eines Traumas eine chirurgische Mitbetreuung. Bei Übernahme wiesen $66 \%$ der Patienten eine Exsikkose auf und $20 \%$ entwickelten eine akute Nierenfunktionsstörung. $58 \%$ der Patienten zeigten eine deutliche Erhöhung der Kreatininkinase (>1000 U/L). Rund $70 \%$ der Patienten mussten intensivmedizinisch weiterbetreut werden. Die mittlere Intensivaufenthaltsdauer betrug $10 \pm 10 \mathrm{~d}$ (Median: $5 \mathrm{~d}$, Spannweite 1-35 d) und die innerklinische Mortalität lag bei $50 \%$ (• Abb. 2).

Zusammenfassung: Patienten mit LT benötigten in dieser Kohorte häufig eine intensivmedizinische Behandlung und wiesen eine hohe Krankenhausmortalität auf. Aufgrund der vielen assoziierten Diagnosen und des 


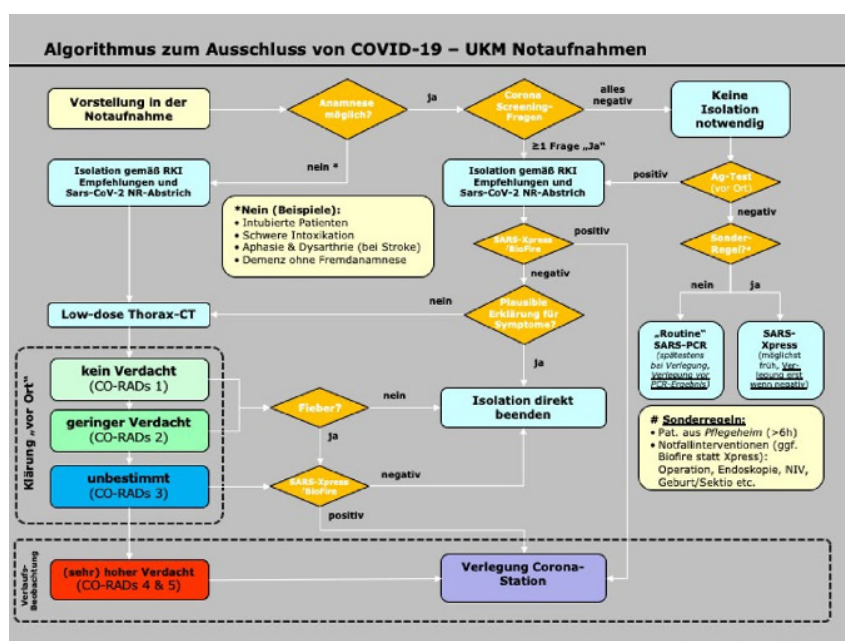

Abb. 1 | P39 4

schlechten Behandlungsergebnis erscheint eine interdisziplinäre Schockraumversorgung von Patienten mit Liegetrauma sinnvoll.

Basierend auf den Erkenntnissen dieser Untersuchung beschreibt das Liegetrauma „ein komplexes klinisches Bild, indem durch vielfältige Ursachen plötzlich das eigenständige Mobilisieren und Hilfe holen verhindert wird und hierdurch weitere Gesundheitsschäden entstehen".

\section{P39}

\section{Algorithmus zum Ausschluss von COVID-19 unter Einbeziehung einer Multiplex PCR in der Notaufnahme}

\section{O. Wennmann, M. Hennies ${ }^{1}$, H. Van Aken ${ }^{2}$, P. Kümpers}

Sektion Interdisziplinäre Notaufnahme, Medizinische Klinik D, Westfälische Wilhelms-Universität Münster, Münster; ${ }^{1}$ Institut für Virologie, Universitätsklinikum Münster, Münster; ${ }^{2}$ Klinik und Poliklinik für Anästhesiologie und operative Intensivmedizin, Universitätsklinikum, Westfälische Wilhelms-Universität Münster, Münster;

Hintergrund: Die COVID-19 Pandemie stellt die Notaufnahme vor die Herausforderung SARS-CoV2 positive Patienten zügig zu identifizieren, um Erkrankte adäquat zu versorgen und weitere Patienten sowie Mitarbeiter effektiv zu schützen. Herausfordernd ist insbesondere die Erkennung nicht anamnestizierbarer Patienten, beispielsweise auf Grund einer Vigilanzminderung. Hierzu haben wir neben Antigen-Schnelltest, regulären PCR Tests im Labor und bildgebenden Verfahren, die Möglichkeit einer point-of-care Testung mittels Multiplex-PCR (Biofire $^{\circledR}$ Filmarray ${ }^{\star}$ ) zum Nachweis der häufigsten respiratorischen Erreger inklusive SARS-CoV-2 in der Notaufnahme etabliert.

Methode: Alle asymptomatischen Patienten werden mittels Antigenschnelltest vor Ort getestet. Die Testung erfolgt unter einer Sicherheitswerkbank. Bei stationärer Aufnahme erfolgt zusätzlich eine PCR Testung im Labor. Symptomatische Patienten werden isoliert und tagsüber ebenfalls im Labor getestet (turn-around time $\sim 4 \mathrm{~h}$ ). Wir nutzen das Biofire ${ }^{\circ}$ Filmarray ${ }^{\oplus}$ insbesondere bei symptomatischen Patienten außerhalb der Kernarbeitszeit und/oder bei nicht sicher anamnestizierbaren Notfallpatienten, die zügig eine Intervention (z. B. NIV, Intubation, Coronarangiographie) benötigen und damit eine kritische Infrastruktur des Krankenhauses nutzen müssen (turn-around time $\sim 45 \mathrm{~min}$.). Nicht anamnestizierbare Patienten mit Infektionszeichen oder einer anderen Indikation zur Bildgebung erhalten zusätzlich zur PCR Testung ein thorakales low-dose CT zur systematischen Beurteilung der Lunge.

Ergebnisse: Dieser Algorithmus wurde am 01.05.2020 in Kraft gesetzt und ist seitdem nur geringfügig angepasst worden (Ag-Test ergänzt 02/21; - Abb. 1)

Insgesamt wurden bislang 1400 Biofire $^{\circledR}$ Filmarray ${ }^{\circledR}$ Teste in der Notaufnahme durchgeführt. Unter den im Untersuchungszeitraum stationär über

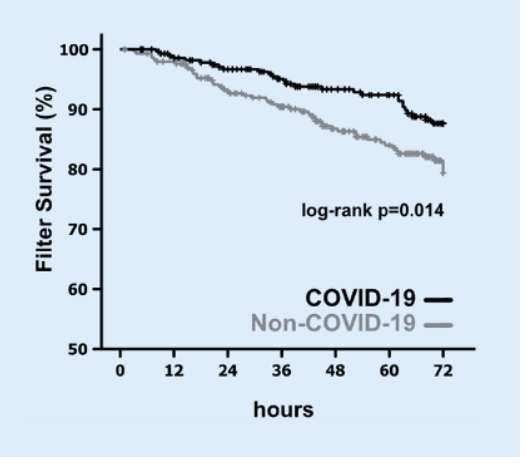

Fig. $1 \mid$ P40 Cumulative CRRT filter survival

die Notaufnahme aufgenommen Patienten (ca. 9500 Fälle) kam es nur zu einer einzigen Eintragung von SARS-CoV-2 ins Klinikum. Fälle von SARS-CoV-2 unter Mitarbeitern der Notaufnahme sind nicht aufgetreten. Bei Notfallpatienten mit zusätzlichen Symptomen eines Atemwegsinfektes können mittels Biofire ${ }^{\oplus}$ Filmarray direkt eine Vielzahl von alternativen Infektionserregern ausgeschlossen werden (z. B. Influenza, RSV, Parainfluenza).

Zusammenfassung: Der Einsatz der point-of-care Multiplex-PCR als Diagnostikbaustein in einem symptombasierten Testalgorithmus ermöglicht einen schnellen und sicheren Nachweis bzw. Ausschluss einer COVID-19 Erkrankung zu jeder Tageszeit.

\section{Nephrologie}

\section{P40}

\section{Efficacy and complications of regional citrate anticoagulation during continuous renal replacement therapy in critically ill patients with COVID-19}

D. Khadzhynov, U. von den Bergen, R. Körner', J.M. Kruse' , D. Zickler', K. Budde, K.-U. Eckardt, L. Lehner

Medizinische Klinik mit Schwerpunkt Nephrologie und Internistische Intensivmedizin, Campus Charité Mitte, Charité - Universitätsmedizin Berlin, Berlin; 'Medizinische Klinik mit Schwerpunkt Internistische Intensivmedizin und Nephrologie, Campus Virchow-Klinikum, Charité - Universitätsmedizin Berlin, Berlin

Objective: Enhanced coagulation in coronavirus disease 2019 (COVID-19) patients is considered a major obstacle for continuous renal replacement therapy (CRRT), but systematic analyses are sparse. We compared filter survival and citrate-induced complications during CRRT with regional citrate anticoagulation (RCA) in COVID-19 and Non-COVID-19 patients. Method: In this retrospective study we included all consecutive adult patients $(n=97)$ with acute kidney injury (AKI) treated with RCA-CRRT at seven ICUs of a tertiary university hospital over the tree month period. Medical data were collected to compare the efficacy and complications of RCA-CRRT between COVID-19 $(n=44)$ and Non-COVID-19 patients $(n=53)$.

Results: There was no significant difference in the number of CRRT filters used per patient in COVID-19 vs. Non-COVID-19 patients (median 5 vs 3 filters, $p=0.103$ ). Mean filter run-time was significantly higher in COVID-19 patients compared to Non-COVID-19 patients $(68.4(95 \% \mathrm{CI}$ 67.0-69.9) vs. 65.2 (95\%CI 63.2-67.2) hours, respectively; log-rank 0.014, - Fig. 1). COVID-19 patients showed significantly higher activated partial thromboplastin time (aPTT) throughout the CRRT due to systemic anticoagulation compared to Non-COVID-19 patients (54 (IQR 45-61) vs. 47 (IQR 41-58) seconds, respectively; $p<0.001$ ). A significantly higher incidence of combined metabolic disturbances (metabolic alkalosis, hypercalcemia and hypernatremia), consistent with reduced filter patency and citrate overload during RCA, was observed in COVID-19 patients 
compared to Non-COVID-19 patients ( $19.1 \%$ vs. $12.7 \%$, respectively; $p=0.04)$. These metabolic disarrangements were resistant to per-protocol adjustments and disappeared after replacement of the CRRT-filter. Conclusion: In contrast to initial concerns, adequate filter life-span can be achieved with RCA during CRRT in COVID-19 patients. However, close monitoring of the acid-base balance appears warranted, as these patients tend to develop reduced filter patency leading to a higher incidence of citrate overload and metabolic disturbances.

\section{P41}

Impact of saline infusion during acute hypotensive events at donor plasmapheresis

\section{J. Evers, U. Taborski'}

Würselen; 'Octapharma Plasma GmbH, Aachen

Objective: The purpose of our research was to evaluate the effects of saline infusion to counteract incidents of acute hypotension during donor plasmapheresis (DP), and on immunoglobulin G (IgG) content in collected plasma, and on donor hemoglobin ( $\mathrm{Hgb}$ ) and hematocrit $(\mathrm{Hct})$ values. Method: IIt is based on three studies: an observation of saline infusion in donors with severe drops in blood pressure at DP, and two crossover trials of 32 repeat plasma donors each on saline $500 \mathrm{ml}$ infusion during DP. DP was performed with the automated plasma collecting system $\left(\mathrm{PCS}^{\circledR} 2\right.$, Haemonetics) via a single needle technique. It operates in a discontinuous flow manner with 4-5 repetitive cycles of draw and collection phases alternating with return phases. Tri-sodium citrate $4 \%$ (TSC) is added in the withdrawal phase in a low flow ratio of 1: 16 to blood at the inlet line to the tubing set prior to the apheresis centrifuge. Separation of the plasma takes place in a disposable bowl by centrifugation at $6000 \mathrm{rpm}$. Most of TSC is retained in the separated plasma, the rest is re-infused to the donor. Note that extracorporeal volume increases during DP to the plasma already collected up to $800-1100 \mathrm{ml}$ including $\mathrm{AC}$ at its maximum before the return phase of the last cycle.

Results: We observed 50 cases with a significant acute drop of mean systolic blood pressure by $45 \pm 16 \mathrm{~mm} \mathrm{HG}$ in one year (incidence $0.15 \%$ ), predominantly in young, female first-time donors. After treatment with placing the donor in the Trendelenburg position along with rapid infusion of $1000 \mathrm{ml}$ saline $49 \pm 18 \mathrm{~min}$ after the event all plasma donors could safely leave the center. In trial 1, there was a reduction of IgG concentration in collected plasma related to the average IgG serum concentration without saline to $85.5 \%$ vs. to $80.5 \%$ with saline $(p<0.001)$. In trial 2 , there was an important $11-12 \%$ increase of the donor hematological values without saline during DP. The difference between the two samples without saline and with saline after the end of DP at $1 \mathrm{~h}$ of $\pm 5.3 \%$ was highly significant

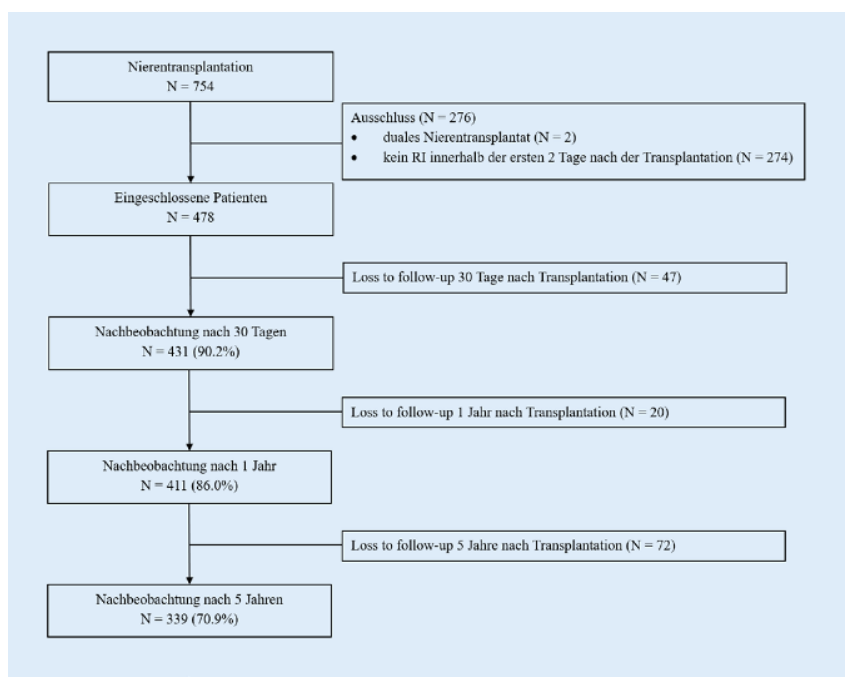

Abb. 1 |P42 A $(p<0.001)$. After $24 \mathrm{~h}$ all differences were reduced by half and after $48-72 \mathrm{~h}$ $\mathrm{Hgb}$ and Hct values returned to baseline.

Conclusion: Our research has led to significant improvements in the practice of DP: 1) Saline should be infused at DP as clinically indicated in agreement with the donor. 2) Young, female first-time donors require special care. 3) Due to the crossover design of the two trials with saline, the impact or level of evidence is high (estimated 1b).

\section{P42}

Vorhersagewert des Renal Resistive Index in der unmittelbaren postoperativen Phase nach Nierentransplantation auf kurz- und langfristige Ergebnisse: ein Update

S. Bogaert, N. Suchonos, A. Decruyenaere' ${ }^{1}$ P. Decruyenaere' ${ }^{1}$, P. Vignesh, T. Westhoff, F. Vermassen', P. Peeters', E. Hoste'

Ruhr Universität Bochum, Bochum; 'Universität Gent, Gent/BE;

Hintergrund: Wir wollten der Vorhersagewert des Renal Resistive Index (RI), der routinemäßig in der unmittelbaren Posttransplantationsphase gemessen wird, zur verzögerten Transplantatfunktion (DGF) in seinen verschiedenen Definitionen und zu Langzeitergebnissen in einer großen Kohortenstudie untersuchen.

Methode: Wir haben eine retrospektive Studie in einem akademischen Zentrum für Tertiärversorgung durchgeführt. Der RI wurde innerhalb der ersten 48 Stunden nach der Nierentransplantation durch Doppler-Sonographie gemessen. Das kurzfristige Ergebnis wurde anhand von 22 Definitionen der DGF untersucht (• Abb. 1).

Ergebnisse: Wir haben 478 Nierentransplantationsempfänger eingeschlossen und konnten 339 (70.9\%) bis 5 Jahre postoperativ nachuntersuchen. Abhängig von der verwendeten Definition war DGF bei 4,4 bis $54,0 \%$ der Empfänger vorhanden. Wir fanden heraus, dass DGF in $50 \%$ der verschiedenen Definitionen mit einem signifikant höheren RI assoziiert war, insbesondere wenn es auf Dialyse beruhte $(85,7 \%)$. Eine univariate CoxRegressionsanalyse ergab, dass ein $\mathrm{RI} \geq 0,70$ ein signifikanter Prädiktor für Gesamtmortalität und Tod mit einem funktionierenden Transplantat, jedoch nicht für ein Versagen des Transplantats war.

Zusammenfassung: Der an den ersten Tagen nach der Nierentransplantation routinemäßig gemessene RI ist kurzfristig mit der Nierenfunktion assoziiert, langfristig jedoch nicht. Ein erhöhter RI ist mit einer Langzeitmortalität korreliert.

\section{P43}

MR-pro-Adrenomedullin als Prädiktor für Notwendigkeit einer Nierenersatztherapie bei kritisch kranken Patienten mit COVID-19

D. Jarczak, K. Roedl, M. Fischer, O. Boenisch, G. de Heer, D. Frings, B. Sensen, M. Karakas, S. Kluge, A. Nierhaus

Klinik für Intensivmedizin, Zentrum für Anästhesiologie und Intensivmedizin, Universitätsklinikum Hamburg-Eppendorf, Hamburg;

Hintergrund: Die Coronaviruserkrankung 2019 (COVID-19) führte zu einer großen Zahl kritisch kranker Patienten, die auf einer Intensivstation (ICU) behandelt wurden. Etwa $20 \%$ dieser Patienten benötigten eine Nierenersatztherapie. Aktuell ist unklar, welche Marker oder Charakteristika ein akutes Nierenversagen (ANV) bzw. die Notwendigkeit einer Nierenersatztherapie valide vorhersagen können. Hier könnten neue Biomarker, wie mid-regional pro-adrenomedullin (MR-proADM) durch ihre Beteiligung bei der Entwicklung eines Multiorganversagens eine Rolle spielen. Ziel dieser Studie war die Untersuchung, ob MR-proADM die Notwendigkeit einer Nierenersatztherapie bei kritisch kranken Patienten mit COVID-19 vorhersagen kann.

Methode: Wir untersuchten alle Patienten mit bestätigter COVID-19 Erkrankung, die konsekutiv auf einer der 12 ICUs einer deutschen Universitätsklinik im Zeitraum März bis September 2020 behandelt wurden. Es wurden klinische Charakteristika und Daten zum Vorkommen eines ANV, der Nierenersatztherapie und zum Überleben erhoben. MR-proADM wurde im Rahmen der klinischen Routine bei Aufnahme gemessen. 
Ergebnisse: 64 Patienten konnten in die Erhebung eingeschlossen werden. Das mediane Alter betrug 62.5 (54-73) Jahre; 49 Patienten (77\%) waren männlich. 50 (78\%) Patienten benötigten eine Vasopressor-Therapie, 47 $(73 \%)$ wurden mechanisch beatmet und 25 (39\%) litten an einem schweren ARDS. Eine venovenöse extrakorporale Membranoxygenierung wurde bei 10 Patienten eingesetzt. 29 (45\%) Patienten benötigten eine Nierenersatztherapie während des ICU-Aufenthaltes; die mediane Zeit von ICU-Aufnahme bis Start der Nierenersatztherapie war 2 (1-9) Tage. MRproADM bei Aufnahme auf die ICU war bei diesen Patienten signifikant erhöht ( 2.491 vs. $1.230 \mathrm{nmol} / \mathrm{l} ; p=0.002)$ und zeigte die beste Korrelation mit dem SOFA-Subscore Niere an Tag 1. Die ROC-Analyse ergab, dass MR-proADM bei ICU-Aufnahme mit einer AUC von 0.685 (95\% CI:0.543-0.828; $p=0.019)$ die Notwendigkeit einer Nierenersatztherapie vorhersagen konnte. In der multivariablen Regression war MR-proADM hierfür ein unabhängiger Prädiktor (OR:3.813; 95 \% CI:1.110-13.102; $p<0.05)$.

Zusammenfassung: Das ANV mit Notwendigkeit einer Nierenersatztherapie ist bei kritisch kranken Patienten mit COVID-19 relativ häufig. MRproADM bei Aufnahme auf die ICU zeigte eine gute Vorhersagekraft für die Notwendigkeit einer Nierenersatztherapie, was für eine frühe Risikostratifizierung und das weitere Management von Bedeutung sein könnte.

\section{Gastroenterologie, Hepatologie und Ernährung}

\section{P44 \\ Schwere Leberfunktionsstörung bei kritisch kranken Patienten mit COVID-19 \\ K. Roedl, D. Jarczak, A. Drolz' ' D. Wichmann, G. de Heer, C. Burdelski, A. Nierhaus, M. Lütgehetmann ${ }^{2}$, S. Kluge, V. Fuhrmann \\ Klinik für Intensivmedizin, Zentrum für Anästhesiologie und Intensivmedizin, Universitätsklinikum Hamburg-Eppendorf, Hamburg; 1 1. Medizinischen Klinik und Poliklinik, Universitätsklinikum Hamburg- Eppendorf, Hamburg; ${ }^{2}$ Institiut für Medizinische Mikrobiologie, Virologie und Hygiene, Universitätsklinikum Hamburg-Eppendorf, Hamburg;}

Hintergrund: Im Rahmen einer Coronavirus Erkrankung (COVID-19) ist das Auftreten einer Leberschädigung relativ häufig. Bei kritisch kranken ist Häufigkeit des Auftretens schwerer Leberfunktionsstörungen unklar. Ziel war es dies genauer zu untersuchen und klinische Charakteristika und Risikofaktoren für das Auftreten zu beschreiben.

Methode: Klinischen Charakteristika und Krankheitsverläufe von konsekutiven allen Patienten mit bestätigter COVID-19 Infektion unserer Klinik behandelt wurden eingeschlossen. Als Studienzeitraum wurde März 2020 bis Juli 2020 definiert. Eine schwere Leberfunktionsstörung wurde folgendermaßen definiert: Bilirubin $\geq 2 \mathrm{mg} / \mathrm{dl}$ und/oder massiver Anstieg der Transaminasen( $>20$-Fach über Norm)

Ergebnisse: Von 72 kritisch kranken Patienten mit COVID-19 entwickelten 22 (31\%) eine schwere Leberfunktionsstörung. Patientencharakteristika (u.a. Alter, Geschlecht, Komorbiditäten) zeigten sich gleich verteilt. Patienten mit schwerer Leberfunktionsstörung zeigten eine schlechtere respiratorische Funktion als Patienten ohne Leberfunktionsstörung $\left(\mathrm{paO}_{2} /\right.$ $\mathrm{FiO}_{2:} 82(58-114)$ vs. $\left.117(83-155) ; p<0.05\right)$. Aus diesem Grund benötigten diese eine entsprechend häufigere mechanische Beatmung( $95 \%$ vs. $64 \%$; $p<0.01)$, Kreislaufunterstützung $(95 \%$ vs. $72 \%$; $p<0.05)$ und Nierenersatztherapie $(86 \%$ vs. $30 \%$; $p<0.001)$. Zudem zeigte sich auch eine höhere Erkrankungsschwere(SAPS II: 48 (39-52) vs. 40 (32-45); $p<0.01$ ). Bei Patienten mit schwerer Leberfunktionsstörung fand sich in $68 \%$ eine Virämie, im Vergleich zeigte sich dies nur bei $34 \%$ ohne Leberfunktionsstörung ( $p=0.002)$. Das Auftreten einer schweren Leberfunktionsstörung war in der logistischen Regressionsanalyse unabhängig mit dem Auftreten einer Virämie [OR 6.359; $95 \%$ CI 1.336-30.253;p<0.05] und mit der Schwere der Erkrankung(SAPS II) assoziiert [OR 1.078; $95 \%$ CI $1.004-$ $1.157 ; p<0.05]$. Das Überleben bei Patienten mit schwerer Leberfunkti- onsstörung war im Vergleich zu Patienten ohne Leberfunktionsstörung deutlich niedriger( $31 \%$ vs. $84 \%, p<0.001$ ). In der Cox-Regressionsanalyse zeigte sich das Auftreten einer schweren Leberfunktionsstörung als unabhängiger Prädiktor für Mortalität [HR 3.347; 95 \% CI 1.401-7.999; $p<0.01$ ]. Zusammenfassung: Bei COVID-19 ist eine schwere Leberfunktionsstörung relativ häufig, das Auftreten ist mit der Präsenz von Virämie und der Schwere der Erkrankung vergesellschaftet.

\section{P45}

Akut-auf-chronisches Leberversagen verändert nicht die Pharmakokinetik bei kritisch kranken Patienten mit kontinuierlichem Nierenersatzverfahren: eine prospektive Beobachtungsstudie

J. Grensemann, C. Pfaffendorf' ', S. Wicha ', C. König ${ }^{2}$, K. Roedl, D. Jarczak, S. Iwersen-Bergmann ${ }^{3}$, C. Manthey, S. Kluge, V. Fuhrmann

Klinik für Intensivmedizin, Zentrum für Anästhesiologie und Intensivmedizin, Universitätsklinikum Hamburg-Eppendorf, Hamburg; ${ }^{1}$ Institut für Pharmazie, Fachbereich Chemie, Universität Hamburg, Hamburg; ${ }^{2}$ Krankenhausapotheke, Universitätsklinikum Hamburg-Eppendorf, Hamburg; ${ }^{3}$ Institut für Rechtsmedizin, Universitätsklinikum HamburgEppendorf, Hamburg;

Hintergrund: Infektionen mit Sepsis sind eine Hauptursache für akut-aufchronisches Leberversagen (ACLF). Diese können neben Bakterien auch durch Schimmelpilze verursacht werden. Die Behandlung erfolgt zumeist mit Voriconazol (VRC), allerdings kann die Pharmakokinetik (PK) durch ACLF verändert werden. Da ACLF-Patienten häufig unter gleichzeitigem akutem Nierenversagen leiden, untersuchten wir die PK von VRC bei Patienten, die eine kontinuierliche Nierenersatztherapie (RRT) mit ACLF und ohne gleichzeitiges Leberversagen (NLF) erhielten.

Methode: In dieser prospektiven Kohortenstudie erhielten die Patienten gewichtsadaptiert VRC. Die Proben wurden mittels Hochleistungsflüssigkeitschromatographie in Kombination mit Fluoreszenz- und Dioden-

\begin{tabular}{|c|c|c|}
\hline Pationt characteristics & no & $\%$ \\
\hline Total patients & 70 & \\
\hline Age - median (range) & $59(18-72)$ & \\
\hline \multicolumn{3}{|l|}{ median (rango) } \\
\hline Fomale sox & 35 & 50 \\
\hline \multicolumn{3}{|l|}{ Indication alloscT } \\
\hline Acute leukemia + MDS & 54 & 77.1 \\
\hline NHL & 10 & 14,3 \\
\hline MPN & 4 & 5,7 \\
\hline Other" & 2 & 2,9 \\
\hline \multicolumn{3}{|l|}{ Remission pre alloSCT } \\
\hline CR & 24 & 34,3 \\
\hline PR & 19 & 27,1 \\
\hline SD & 4 & 5,7 \\
\hline PD & 16 & 22,9 \\
\hline Molelucar relapse & 7 & 10,0 \\
\hline $\mathrm{HCT}-\mathrm{Cl}$ - median (rango) & $4(0-10)$ & \\
\hline \multicolumn{3}{|l|}{ Type of donor } \\
\hline Haplo & 9 & 12,9 \\
\hline MMUD & 15 & 21,4 \\
\hline MUD & 39 & 55,7 \\
\hline sib & 7 & 10,0 \\
\hline Acute GvHD & 36 & 51,4 \\
\hline Grade $1 / 2$ & 26 & 72,2 \\
\hline Grado $3 / 4$ & 10 & 27,8 \\
\hline * 1x NK-cell leukemia; $1 \mathrm{x}$ & & \\
\hline
\end{tabular}

Fig. 1 | P46 $\Delta$ 


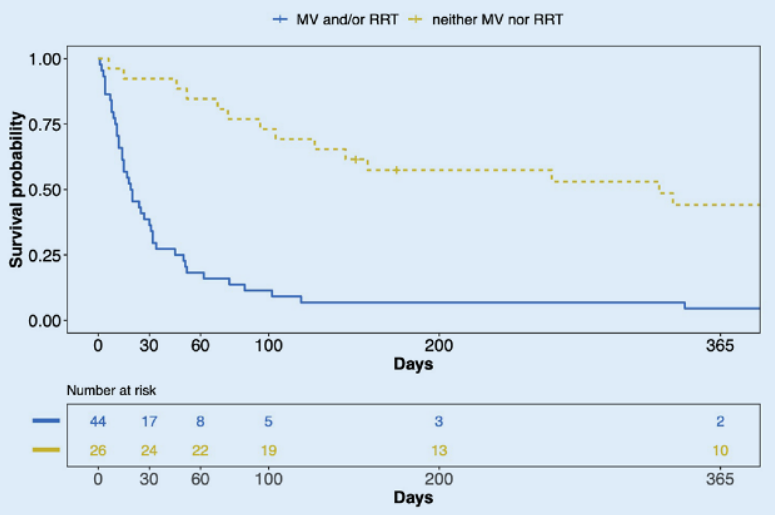

Fig. 2 | P46

array-Detektoren analysiert. Die PK wurde mittels einem integrierten pharmakometrischen Modell analysiert. Mit dem Modell wurden die Standarddosis, eine um $50 \%$ niedrigere und $50 \%$ höhere Dosis mittels Monte-Carlo-Simulation (MCS) für Tag 1 und im Steady-State mit einer angestrebten Talkonzentration (TC) von $0,5-3 \mathrm{mg} / \mathrm{L}$ simuliert.

Ergebnisse: Vierzehn Patienten wurden in diese Studie eingeschlossen, davon 6 Patienten mit ACLF. Ein Zwei-Kompartiment-Modell mit linearer Clearance beschrieb die PK von VRC. Es bestand kein Unterschied für das zentrale (V1) oder periphere (V2) Verteilungsvolumen oder die Clearance zwischen den beiden Gruppen. Das V1 betrug 80,6 L (95 \% Konfidenzintervall: 62,6-104) und V2 $106 \mathrm{~L}$ (65-166) mit einer Körper-Clearance von $4,7 \mathrm{~L} / \mathrm{h}(2,87-7,81)$ und einer RRT-Clearance von $1,46 \mathrm{~L} / \mathrm{h}(1,29-1,64)$. Nach MCS lag die TC für die Standarddosis an Tag 1 unter/innerhalb/ oberhalb des Zielwerts zu 10/74/16\% und zu 9/39/52 \% im Steady-State. Eine $50 \%$ niedrigere Dosis führte zu 26/72/1\% (Tag 1) und 17/64/19\% im Steady-State und eine $50 \%$ höhere Dosis zu 7/57/37 \% und 7/27/67 \%. Zusammenfassung: Die PK von VRC wird durch ACLF bei kritisch kranken Patienten, die eine RRT erhalten, nicht relevant beeinflusst. Die Erhaltungsdosis sollte in beiden Gruppen angepasst werden. Aufgrund der hohen interindividuellen Variabilität erscheint ein therapeutisches Drug Monitoring unumgänglich.

\section{Hämatoonkologische Intensivmedizin}

\section{P46}

\section{Characteristics and outcomes of allogeneic stem cell transplant recipients admitted to the intensive care unit within $\mathbf{3 0}$ days from transplantation - A retrospective analysis from a German University Hospital}

J. Garcia Borrega, J.-M. Heger, P. Köhler, U. Holtick, M. Hallek, C. Scheid, M. Kochanek, B. Böll, A. Shimabukuro-Vornhagen, D. Eichenauer Klinik für Innere Medizin I, Universitätsklinikum Köln, Köln;

Objective: The overall prognosis of allogeneic stem cell recipients admitted to the intensive care unit (ICU) has improved over the last decades. However, analyses focusing on patients treated on the ICU during the peritransplant period are scarce. We therefore conducted a retrospective study comprising 70 patients who underwent allogeneic stem cell transplantation (aSCT) at our institution between 2014 and 2020 and were admitted to the ICU during conditioning therapy or within 30 days from transplantation.

Method: Between 2014 and 2020, 638 patients underwent aSCT at our institution. Among those, 70 individuals (11\%) were admitted to the ICU between the start of conditioning therapy and day 30 after aSCT and were thus eligible for the present analysis. Patient characteristics were extract- ed from patients charts and analyzed descriptively. The influence of single variables on overall survival was compared using Log-Rank (Mantel-Cox) test. Statistical significance was set at $p<0.05$. Survival was calculated using the Kaplan-Meier method.

Results: Patient characteristics are shown in • Fig. 1.

The median age was 59 years (range: $18-72$ years). $50 \%$ of patients were female. Sepsis was the most common cause for ICU admission (49\%). $56 \%$ of patients required mechanical ventilation (MV) and $27 \%$ received renal replacement therapy (RRT). The ICU, 60-day, hospital and 1-year survival rates were $48.6 \%, 41.4 \%, 38.6 \%$ and $16.2 \%$, respectively. MV and/ or RRT during the stay on the ICU were associated with an impaired sur$\operatorname{vival}(p=0.001)$ ( Fig. 1). Patients with progressive disease at the time of aSCT had significantly impaired survival as well $(p=0.006)$ Baseline characteristics such as age and gender did not impact the outcome Only 2 of 39 patients who required MV were alive at 1 year. None of the patients in whom cardiopulmonary resuscitation $(n=12)$ or RRT $(n=19)$ was necessary were alive at 1 year.

Among patients who died after discharge from the ICU $(n=23)$, infectious complications represented the major cause of death (48\%). Relapse of the underlying disease and graft-versus-host disease accounted for $17 \%$ and $13 \%$ of deaths, respectively.

Conclusion: The present analysis indicates unfavorable long-term outcomes for allogeneic stem cell recipients admitted to the ICU during the peri-transplant period. The need for organ failure support using MV and RRT displays the major risk factor for impaired survival. These results might offer advantages regarding informed decision making with patients and their families.

\section{Qualitätssicherung/ Kostenevaluierung}

\section{P47}

Veränderung der Verweildauer von Patienten mit Influenza durch Etablierung einer Multiplex-PCR (mPCR)-Testung in der Notaufnahme

D. O. Wennmann, M. Hennies', P. Kümpers

Sektion Interdisziplinäre Notaufnahme, Medizinische Klinik D, Westfälische Wilhelms-Universität Münster, Münster; 'Institut für Virologie, Universitätsklinikum Münster, Münster;

Hintergrund: Die Versorgung von Patienten mit Atemwegsinfektionen stellt in der Notaufnahme eine Herausforderung dar, da potentiell infektiöse Patienten isoliert werden müssen. Patienten mit Indikation zur stationären Aufnahme müssen auf Normal- und Infektionsstation verteilt werden. Die schnelle Identifizierung eines Erregers kann eine ressourcenschonende Versorgung ermöglichen. Diese Arbeit untersucht am Beispiel der Influenza, ob eine mPCR Testung vor Ort in der Notaufnahme einen Einfluss auf die Zeit von Aufnahme bis zur stationären Verlegung bzw. Entlassung aus der Notaufnahme hat. Mittels der mPCR können Patienten innerhalb von 45 min auf eine Vielzahl von Erregern (z. B. Influenza, Parainfluenza, RSV-Virus, SARS-CoV2, Mykoplasmen) zuverlässig untersucht werden. Methode: Seit dem Jahreswechsel 2019-2020 steht zur mPCR Testung das Biofire $^{\oplus}$ Filmarry ${ }^{\circledR}$ in den Räumlichkeiten der Notaufnahme des Universitätsklinikums Münster (UKM) zur Verfügung. Die Patientendaten der Jahre 2018 bis 2021 wurden auf den Nachweis einer Influenza Infektion mittels retrospektiver Datenbankabfrage, KIS und Labor-Software, untersucht. Vor dem Jahr 2020 erfolgte die Testung vorwiegend im Labor der Virologie des Universitätsklinikums. Am Beispiel der Influenza wurde die durchschnittliche Liegedauer auf der Notaufnahme in den Jahren 2018 und 2019 mit der durchschnittlichen Liegedauer im Jahr 2020 und 2021 verglichen.

Ergebnisse: Insgesamt wurde $n=189$ Patienten mit nachgewiesener Influenza im genannten Zeitraum identifiziert. Die durchschnittliche Liegedau- 
er in der Notaufnahme in den Jahren 2018 und 2019 lag bei 963 Minuten (Median: 991, Q 25 : 489, Q 75 : 1375). Nach Einführung im Jahr 2020 sank die durchschnittliche Liegedauer auf 593 Minuten (Median: 409, $\mathrm{Q}_{25}: 207$, $\left.\mathrm{Q}_{75}: 720, p<0,05\right)$.

Zusammenfassung: Mittels point of care mPCR lässt sich die Verweildauer auf der Notaufnahme für Patienten mit Influenza signifikant reduzieren. Die Methode erlaubt die schnelle (Dauer der PCR 45 Minuten), Tageszeit unabhängige Diagnose einer Infektion der oberen Atemwege und damit die schnelle Zuweisung der Patienten in den stationären Bereich (z. B. Infektionsstation) oder den ambulanten Sektor.

\title{
Pflege
}

\section{P48}

\section{Pflegeaufwandsindizes TISS-28 und INPULS ${ }^{\oplus}$ für die Intensivstation - zwei Systeme im direkten Vergleich (PATIN)}

\author{
A.-K. Tack, F. Glaß' \\ INPULS $^{\circledast}$, Pflegeteam, Universitätsklinikum Heidelberg, Heidelberg; \\ ${ }^{1}$ Chirurgische Klinik, Universitätsklinikum Heidelberg, Heidelberg;
}

Hintergrund: Im Rahmen dieser monozentrischen, quantitativ analytische Beobachtungsstudie soll mittels der Fragestellung „Weist TISS-28 oder INPULS ${ }^{\circledast}$ eine höhere Sensitivität in der Abschätzung des tat-sächlichen Pflegeaufwandes von Patienten einer Intensiv- und/oder Intermediat-CareStation auf?" die Abbildung des Pflegeaufwandes durch die Instrumente TISS-28 und INPULS ${ }^{\circledast}$ unter-sucht werden. Als Nebenzielkriterium wird betrachtet ob ein Zusammenhang zwischen der Krankheitsschwere, der Prognose und dem Pflegeaufwand hergestellt werden kann.

Methode: Betrachtet wurden über einen Zeitraum von 30 Tagen die Patient:innen einer viszeralchirurgischen und anästhesiologischen Intensiv-, sowie einer Intermediate-Care-Station innerhalb eines Hauses der Maximalversorgung. Ein Ausschluss fand sich in den täglichen $\mathrm{Zu}-$ und Abgängen der Patient:innen der jeweiligen Station, sowie in einem Alter von unter 18 Jahren. Die Kollektion der im täglichen Routinebetrieb erhobenen Daten erfolgte retrospektiv. Diese umfassten neben dem TISS-28-Score weitere medizinisch relevante Scores (SAPS-II, APACHE, SOFA) sowie die jeweiligen Eingruppierungen und Informationen aus dem INPULS ${ }^{\circledR}$-System. Des Weiteren wurden gesondert in einem von den Forschenden eigens entwickelten, experimentellen Score patientenbezogene und für die Intensivpflege relevante Indikatoren, mit dem Ziel die tatsächliche Pflegeaufwändigkeit zu objektivieren, erfasst. Die statistische Auswertung der Daten erfolgte mittels des Programmes IBM SPSS (Version 26) und beinhaltete neben der Deskription der erhobenen Daten die Darstellung möglicher Korrelationen der jeweiligen Scores.

Ergebnisse: In die Auswertung der Studiendaten konnten 845 vollständige Datensätze eingeschlossen werden. Eine Analyse der Daten findet derzeit statt, somit liegen noch keine endgültigen Ergebnisse vor. Zum Kongresstermin können indes vorläufige Ergebnisse präsentiert werden.

Zusammenfassung: Im Angesicht der Debatte um eine adäquate Personalbemessung, sowie eine Abbildung der Leistungen der Intensivpflege zeigt sich die Thematik der Studie als hochaktuell. Es bedarf flächendeckende Instrumente zur Abbildung von Pflegeleistungen, welche derweil nicht existieren. Jedoch kann mittels dieser Studie ein Beitrag zur Verwendung und Aussagekraft verschiedener Systeme getätigt werden. 


\section{Autorenverzeichnis}

\begin{tabular}{|c|c|}
\hline A & \\
\hline Adler, C. & P38 \\
\hline Alemdar, F. & P36 \\
\hline Aletaha, D. & P05 \\
\hline Annecke, T. & P01 \\
\hline Aurich, $\mathrm{P}$. & P25 \\
\hline B & \\
\hline Bach, F. & P01 \\
\hline Baltes, P. & P02 \\
\hline Bauer, M. & P01 \\
\hline Bécède, $M$. & P05 \\
\hline Beckmann, L. & P06 \\
\hline Bellgardt, M. & P34 \\
\hline Bernhard, M. & P38 \\
\hline Beutel, G. & P08 \\
\hline Bode, C. & P07 \\
\hline Boenisch, 0. & P43 \\
\hline Bogaert, S. & P42 \\
\hline Bojic, A. & P30 \\
\hline Böll, B. P06, & P46 \\
\hline Bota, M. & P02 \\
\hline Both, A. & P03 \\
\hline Brand, M. & P36 \\
\hline Brenner, T. & P01 \\
\hline Brinkmann, A. & P01 \\
\hline Brockmann, A. & P29, P33 \\
\hline Bruder, 0. & P25 \\
\hline Budde, K. & P40 \\
\hline Burdelski, C. & P44 \\
\hline Burst, V. & P10, P38 \\
\hline Büttner, S. & P15 \\
\hline
\end{tabular}

\section{D}

$\begin{array}{ll}\text { Daubmann, A. } & \text { P32 } \\ \text { David, S. } & \text { P07 } \\ \text { de Bakker, H. } & \text { P36 } \\ \text { de Heer, G. } & \text { P43, P44 } \\ \text { Debl, K. } & \text { P13 } \\ \text { Decruyenaere, A. } & \text { P42 } \\ \text { Decruyenaere, P. } & \text { P42 } \\ \text { di Cristanziano, V. } & \text { P06 } \\ \text { Dienemann, T. } & \text { P13 } \\ \text { Domanovits, H. } & \text { P16 } \\ \text { Drolz, A. } & \text { P44 } \\ & \\ \text { E } & \\ \text { Eberl, I. } & \\ \text { Eckardt, K.-U. } & \text { P40 } \\ \text { Eibensteiner, F. } & \text { P16, P27 } \\ \text { Eichenauer, D. } & \text { P06, P46 } \\ \text { Evers, J. } & \text { P41 }\end{array}$

$\mathrm{F}$

Felfernig, M. P37

Fichte, J. P31

Fischer, $\mathrm{H}$. $\quad$ P37

Fischer, M. P32, P43

Fischer, $U$. $\quad$ P18

Frank, T. P10

Frey, $\mathrm{O}$ P01, $\mathrm{P03}$

Frings, D. $\quad$ P32, P43

Fuhrmann, V. $\quad$ P44, P45

Fuhrmann, $V . \quad$ P35

G

Garcia Borrega, J. P06, P46

Gärtner, M. P31

Gathoff, B. P06

Geibel, M. P11

Geis, F. P15

Gilmour, S. P33

Gjolli, J. P28

Glaß, F. P48

Grafeneder-

Weissteiner, T. $\quad$ P30

Grensemann, J. $\quad$ P29, P33, P45

Gronski, P.A. P07

Gunga, H.-C. P17

Günther, U. P34

\section{$\mathrm{H}$}

Haap, M. P19

Hackl, M. P10, P38

Hagel, $S . \quad P 01$

Hagmann, $\mathrm{H}$. P06

Hallek, M. P46

Haller, $\mathrm{H}$. $\quad \mathrm{P07}$

Heger, J.-M. P46

Heinz, G. P05

Hempel, D. P14

Hennies, M. P39, P47

Herkner, $\mathrm{H}$. P35

Hermann, A. P30

Herold, J. P15

Hillebrand, C. $\quad$ P05

Hillebrand, U.C. $\quad$ P07

Hippchen, T. $\quad P 09$

Hoeper, M. $\quad P 07$

Hoffmann, C. P20, P21

Hohn, A. P01

Holtick, U. P46

Hopf, H.-B. P31

Hoste, E. P42

Hüser, C. P10, P38

I

Iwersen-Bergmann, S. P45

\begin{tabular}{|c|c|}
\hline \multicolumn{2}{|l|}{ J } \\
\hline Jaksits, S. & P05 \\
\hline Janke, D. & P17 \\
\hline Janssen, G. & P02 \\
\hline Jarczak, D. & P01, P43, P44, P45 \\
\hline Joannidis, M. & P22 \\
\hline Julia, N. & P06 \\
\hline \multicolumn{2}{|l|}{ K } \\
\hline Kagelmann, N. & P17 \\
\hline Kahl, U. & P32 \\
\hline Kampmeier, S. & P04 \\
\hline Karakas, M. & P43 \\
\hline Keuchel, M. & P02 \\
\hline Khadzhynov, D. & P40 \\
\hline Kiehntopf, M. & P01 \\
\hline Kienbacher, $C$. & P35 \\
\hline Kiyan, Y. & P07 \\
\hline Klamt, A.C. & P18 \\
\hline Klein, S. & P22 \\
\hline Kluge, $S$. & P01, P03, P29, P32, P33, P43, \\
\hline & P44, P45 \\
\hline Knafeli, R. & P34 \\
\hline Kochanek, M. & P06, P46 \\
\hline Köhler, P. & P46 \\
\hline König, C. & P03, P45 \\
\hline Körner, R. & $\mathrm{P} 40$ \\
\hline Kruse, J.M. & P40 \\
\hline Kümpers, P. & P04, P12, P14, P39, P47 \\
\hline \multicolumn{2}{|l|}{ L } \\
\hline Lehmann, T. & P01 \\
\hline Lehner, L. & P40 \\
\hline Lemberg, $\mathrm{K}$. & P10 \\
\hline Leschowski, N. & P14 \\
\hline Lim, A. & P09 \\
\hline Link, C. & P11 \\
\hline Lunz, D. & $\mathrm{P} 13$ \\
\hline Lüsebrink, E. & P23, P24 \\
\hline Lütgehetmann, M. & P44 \\
\hline \multicolumn{2}{|l|}{$M$} \\
\hline Machold, K. & P05 \\
\hline Maggioni, M.A. & P17 \\
\hline Manda, D. & P25 \\
\hline Manthey, C. & P45 \\
\hline Markstaller, $\mathrm{K}$. & P05 \\
\hline Massberg, S. & $\mathrm{P} 23, \mathrm{P} 24$ \\
\hline Mayerhöfer, T. & $\mathrm{P} 22$ \\
\hline Meisel, T.S. & P11 \\
\hline Meiser, A. & P11, P34 \\
\hline Merle, U. & P09 \\
\hline Michels, G. & P01 \\
\hline Mighali, M. & P25 \\
\hline Moll, K. & P19 \\
\hline Müller, C. & P01 \\
\hline Müller, R.-U. & P10 \\
\hline
\end{tabular}




\section{Autorenverzeichnis}

\section{N}

Nierhaus, A.

P01, P32, P43, P44

0

Opatz, 0.

Oppenauer, J.

P17

Orban, $M$.

$\mathrm{P} 16, \mathrm{P} 26, \mathrm{P} 27$

P23, P24

\section{P}

Paschen, $\mathrm{C} . \quad \mathrm{P} 30$

Pavenstädt, $\mathrm{H} . \quad \mathrm{P} 04, \mathrm{P} 12$

Peeters, $P$. P42

Pfaffendorf, $C . \quad P 45$

Philipp, A. P13

Pletz, M. P01

R

Rathgeber, I. P18

Reshetnik, A. P28

Riessen, R. $\quad P 19$

Ritzka, M. P13

Robak, $0 . \quad$ P30

Roedl, K. P43, P44, P45

Rohde, $\mathrm{H}$. $\quad \mathrm{P03}$

Röhr, A. P03

Rosenberg, M. P15

Rossaint, J. P04

Roth, D. P35

Rottorf, M. P25

Rovas, A. P04

\section{S}

Sackarnd, J. $\quad$ P04

Sackey, P. P34

Sauer, A. P07

Scheid, C. P46

Schellongowski, P. P05, P30

Schenk, $\mathrm{H}$. $\quad \mathrm{P07}$

Scherbaum, C. P10

Scherer, $C$ P23, P24

Schiehser, G. P37

Schmidbauer, L. P18

Schmidt, B.M.W. P07

Schmidt, J.J. $\quad$ P07

Schnaubelt, S. P16, P26, P27

Schneeweiß-

Gleixner, M.

Schork, A.

Schreiber, W.

Schweiger, S.

SEDACONDA

study group

Seeliger, B.

Sengölge, G.

Sensen, B.

Sharif, A.N.

Shimabukuro-

Vornhagen, $A$.

Sibbing, D.

Sossalla, S.

Spörrer, J.

Stahl, K.
Staudinger, T. $\quad$ P05, P30

Stocker, T. $\quad$ P23, P24

Storm, C. P17

Suárez, V. P10, P38

Suchonos, N. P42

$\mathrm{T}$

Taborski, U. P41

Tack, A.-K. P48

Tappert, I. P10

Tariparast, P.A. P29, P33

Tihanyi, D. P16

Trappe, H.-J. P36

Trauner, M. P05

Tscherny, K. P35

V

Van Aken, $\mathrm{H}$. P39

van der Giet, $M$. P28

van Drongelen, N. P15

Vermassen, $\mathrm{F}$. P42

Vignesh, $P$. P42

Vink, $\mathrm{H}$. P04

Voigt, I. $\quad$ P25

Volk, T. P11, P34

von den Bergen, $\mathrm{U} . \quad \mathrm{P} 40$

W

Wagner, R. P19

Warmbein, A. P18

Weigand, M. P01, P09

Weismann, D. $\quad$ P01

Welker, A. P09

Welte, $T$. P07

Wennmann, D. O. P12, P39, P47

Westhoff, T. P42

Wicha, S. P45

Wichmann, D. P03, P44

Wille, J. P08

Winter, M.-P. P05

Witzke, D. P01

Wörrlein, J. $\quad$ P13

Wygrecka, M. P07

$Y$

Yu, Y. P32

Z

Zauner, C. P05

Zickler, D. P40

Zweckerl, A. P13

Zwickel, P. P15 